\title{
Synthesis of Substituted Carbazoles, Indoles and Dibenzofurans by Vinylic to Aryl Palladium Migration
}

\author{
Jian Zhao and Richard C. Larock* \\ Department of Chemistry, Iowa State University, Ames, Iowa 50011 \\ larock@iastate.edu \\ Table of Contents \\ I. General Procedures \\ S1 \\ II. Noncommercial compounds \\ S2-S4 \\ III. Experimental Procedures \\ S5-S10 \\ IV. Characterization Data for Selected Compounds. S12-S91
}

I. General Procedures. All ${ }^{1} \mathrm{H}$ and ${ }^{13} \mathrm{C}$ spectra were collected in $\mathrm{CDCl}_{3}$ unless noted otherwise. Thin-layer chromatography was performed using 60-mesh silica gel plates, and visualization was effected with short wavelength UV light (254 nm) and a basic $\mathrm{KMnO}_{4}$ solution. High resolution mass spectra were recorded using EI.

All reagents were used directly as obtained commercially unless otherwise noted. Anhydrous forms of acetonitrile, DMF, diethyl ether, ethyl acetate, hexanes, and 4,4-dimethyl-2-pentyne were purchased from Lancaster Synthesis, Inc. 3-Iodoaniline, 2-(trimethylsilyl)phenyl trifluoromethanesulfonate, cesium fluoride, 1,3-diiodobenzene, 1,1'-bis(diphenylphosphino)ferrocene (dppf), sodium tert-butoxide, p-toluidine, $p$-anisidine, $p$-chloroaniline, o-methoxyaniline, methyl 4-aminobenzoate, 1-naphthylamine, 5,6,7,8-tetrahydronaphthalen-1-ylamine, 1-phenyl-1-butyne, 1-phenyl-1-propyne, 4-octyne, diphenylacetylene, 3,5-dimethoxyaniline, sodium thiomethoxide, and trifluoroacetic acid- $d$ were purchased from Aldrich Chemical Co., Inc. Cesium pivalate was prepared according to the procedure of Campo and Larock. ${ }^{1}$ The substituted alkynes were prepared by the Sonogashira coupling of aryl iodides with 1-butyne using $5 \mathrm{~mol} \%$ of $\mathrm{PdCl}_{2}\left(\mathrm{PPh}_{3}\right)_{2}, 2 \mathrm{~mol} \%$ of $\mathrm{CuI}$ in $\mathrm{Et}_{3} \mathrm{~N}$ solvent at room temperature. ${ }^{2}$

\section{Noncommercial compounds.}


$N$-Phenyl-3-iodoaniline. This compound was prepared according to the reported procedure: ${ }^{3} \mathrm{H}$ NMR $\left(\mathrm{CDCl}_{3}\right) \delta 5.67(\mathrm{~s}, 1 \mathrm{H}), 6.94-7.10(\mathrm{~m}, 5 \mathrm{H}), 7.23-7.41(\mathrm{~m}, 4 \mathrm{H}) ;{ }^{13} \mathrm{C} \mathrm{NMR}\left(\mathrm{CDCl}_{3}\right)$ 95.2, 116.5, 119.1, 122.3, 125.9, 129.7, 129.8, 131.1, 142.2, 145.1; IR $\left(\mathrm{CDCl}_{3}\right) 3427,3061,3034,1584 \mathrm{~cm}^{-1} ; \mathrm{HRMS} \mathrm{m} / \mathrm{z}$ 294.9858 (calcd for $\mathrm{C}_{12} \mathrm{H}_{10} \mathrm{NI}, 294.9863$ ).

Other aniline starting materials were prepared through the following palladium-catalyzed amination reaction. $^{4}$ The typical yield is $\sim 30 \%$.

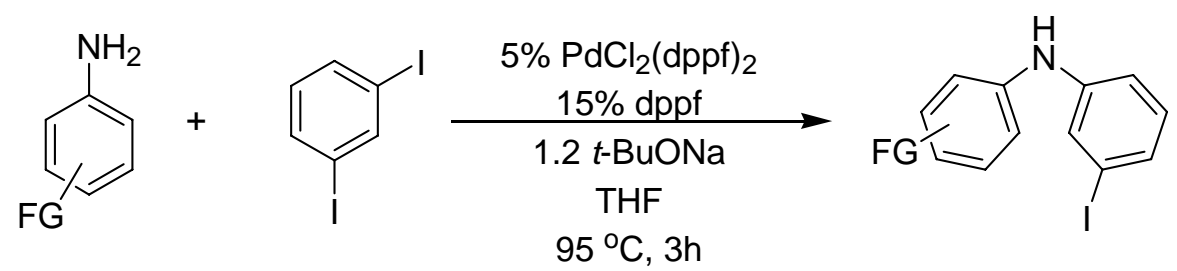

$\boldsymbol{N}$-p-Tolyl-3-iodoaniline. ${ }^{1} \mathrm{H} \mathrm{NMR}\left(\mathrm{CDCl}_{3}\right) \delta 2.34(\mathrm{~s}, 3 \mathrm{H}), 5.58(\mathrm{~s}, 1 \mathrm{H}), 6.93-7.26(\mathrm{~m}, 7 \mathrm{H}), 7.34(\mathrm{~s}$, $1 \mathrm{H}) ;{ }^{13} \mathrm{C} \mathrm{NMR}\left(\mathrm{CDCl}_{3}\right) 21.1,95.3,115.7,120.2,124.9,129.0,130.3,131.0,132.3,139.3,145.9 ; \mathrm{IR}$ $\left(\mathrm{CDCl}_{3}\right) 3427,3028,2922,1587 \mathrm{~cm}^{-1} ; \mathrm{HRMS} \mathrm{m} / \mathrm{z} 309.0018$ (calcd for $\mathrm{C}_{13} \mathrm{H}_{9} \mathrm{IN}, 309.0015$ ).

$N$-(2-Methoxyphenyl)-3-iodoaniline. ${ }^{1} \mathrm{H}$ NMR $\left(\mathrm{CDCl}_{3}\right) \delta 3.89(\mathrm{~s}, 3 \mathrm{H}), 6.15(\mathrm{~s}, 1 \mathrm{H}), 6.92-7.01(\mathrm{~m}, 4 \mathrm{H})$, 7.09-7.12 (m, 1H), 7.26-7.35 (m, $2 \mathrm{H}), 7.51(\mathrm{t}, J=1.7 \mathrm{~Hz}, 1 \mathrm{H}) ;{ }^{13} \mathrm{C} \mathrm{NMR}\left(\mathrm{CDCl}_{3}\right)$ 55.9, 95.2, 111.0, 116.2, 117.2, 121.1, 121.3, 126.5, 129.9, 131.0, 131.9, 144.7, 149.0; IR $\left(\mathrm{CDCl}_{3}\right) 3418,3060,2962,1244$; HRMS m/z 324.9969 (calcd for $\mathrm{C}_{13} \mathrm{H}_{9} \mathrm{INO}, 324.9964$ ).

$N$-(4-Methoxyphenyl)-3-iodoaniline. ${ }^{1} \mathrm{H}$ NMR $\left(\mathrm{CDCl}_{3}\right) \delta 3.83(\mathrm{~s}, 3 \mathrm{H}), 5.51(\mathrm{~s}, 1 \mathrm{H}), 6.82-7.23(\mathrm{~m}, 8 \mathrm{H})$; ${ }^{13} \mathrm{C} \mathrm{NMR}\left(\mathrm{CDCl}_{3}\right)$ 55.9, 95.5, 114.7, 115.1, 123.5, 123.9, 128.3, 131.1, 134.7, 147.1, 156.1; IR $\left(\mathrm{CDCl}_{3}\right)$ 3425, 3006, 2957, $1245 \mathrm{~cm}^{-1}$; HRMS m/z 324.9967 (calcd for $\mathrm{C}_{13} \mathrm{H}_{9} \mathrm{INO}, 324.9964$ ).

Methyl $N$-(3-iodophenyl)benzoate. ${ }^{1} \mathrm{H}$ NMR $\left(\mathrm{CDCl}_{3}\right) \delta 3.88(\mathrm{~s}, 3 \mathrm{H}), 6.10(\mathrm{~s}, 1 \mathrm{H}), 6.98-7.13(\mathrm{~m}, 4 \mathrm{H})$, $7.35(\mathrm{~d}, J=7.8 \mathrm{~Hz}, 1 \mathrm{H}), 7.50(\mathrm{~s}, 1 \mathrm{H}), 7.92(\mathrm{~d}, J=8.7 \mathrm{~Hz}, 1 \mathrm{H}) ;{ }^{13} \mathrm{C} \mathrm{NMR}\left(\mathrm{CDCl}_{3}\right)$ 52.1, 95.0, 115.6, 119.1, 122.2, 128.6, 131.1, 131.8, 131.9, 142.7, 147.3, 167.1; IR $\left(\mathrm{CDCl}_{3}\right) 3340,2945,1694,1580 \mathrm{~cm}^{-1}$; HRMS m/z 352.9913 (calcd for $\mathrm{C}_{14} \mathrm{H}_{12} \mathrm{INO}_{2}, 352.9918$ ). 
$N$-(4-Chlorophenyl)-3-iodoaniline. ${ }^{1} \mathrm{H} \mathrm{NMR}\left(\mathrm{CDCl}_{3}\right) \delta 5.64(\mathrm{~s}, 1 \mathrm{H}), 6.96-7.00(\mathrm{~m}, 4 \mathrm{H}), 7.23-7.28(\mathrm{~m}$, 3H), $7.36(\mathrm{~s}, 1 \mathrm{H}) ;{ }^{13} \mathrm{C} \mathrm{NMR}\left(\mathrm{CDCl}_{3}\right)$ 95.3, 116.8, 120.1, 126.1, 126.8, 129.7, 130.2, 131.2, 140.9, 144.6; IR $\left(\mathrm{CDCl}_{3}\right)$ 3427, 3061, 3034, $1583 \mathrm{~cm}^{-1}$; HRMS m/z 328.9473 (calcd for $\mathrm{C}_{12} \mathrm{H}_{9} \mathrm{ClIN}, 328.9468$ ). $N$-(3-Iodophenyl)naphthalen-1-amine. ${ }^{1} \mathrm{H} \mathrm{NMR}\left(\mathrm{CDCl}_{3}\right) \delta 5.83(\mathrm{~s}, 1 \mathrm{H}), 6.85-6.97(\mathrm{~m}, 2 \mathrm{H}), 7.21$ (dt, $J=7.6,1.3 \mathrm{~Hz}, 1 \mathrm{H}), 7.30(\mathrm{t}, J=1.9 \mathrm{~Hz}, 1 \mathrm{H}), 7.37-7.56(\mathrm{~m}, 4 \mathrm{H}), 7.65(\mathrm{~d}, J=7.8 \mathrm{~Hz}, 1 \mathrm{H}), 7.88-8.00(\mathrm{~m}$, $2 \mathrm{H}) ;{ }^{13} \mathrm{C}$ NMR $\left(\mathrm{CDCl}_{3}\right)$ 95.2, 115.9, 118.2, 122.1, 124.5, 125.3, 126.2, 126.6, 128.6, 128.8, 129.1, 131.0, 134.9, 137.7, 146.9; IR $\left(\mathrm{CDCl}_{3}\right) 3415,3060,1574 \mathrm{~cm}^{-1}$; HRMS m/z 345.0018 (calcd for $\mathrm{C}_{18} \mathrm{H}_{12} \mathrm{IN}$, $345.0015)$

$N$-(3-Iodophenyl)-5,6,7,8-tetrahydronaphthalen-1-amine. ${ }^{1} \mathrm{H} \mathrm{NMR}\left(\mathrm{CDCl}_{3}\right) \delta 1.79-1.90(\mathrm{~m}, 4 \mathrm{H})$, $2.60(\mathrm{t}, J=6.1 \mathrm{~Hz}, 2 \mathrm{H}), 2.84(\mathrm{t}, J=6.1 \mathrm{~Hz}, 2 \mathrm{H}), 5.31(\mathrm{~s}, 1 \mathrm{H}), 6.87-6.90(\mathrm{~m}, 2 \mathrm{H}), 6.96(\mathrm{t}, J=7.7 \mathrm{~Hz}$, 1H), $7.11(\mathrm{~d}, J=4.3 \mathrm{~Hz}, 2 \mathrm{H}), 7.20-7.22(\mathrm{dd}, J=7.6,0.9 \mathrm{~Hz}, 1 \mathrm{H}), 7.30(\mathrm{~s}, 1 \mathrm{H}) ;{ }^{13} \mathrm{C} \mathrm{NMR}\left(\mathrm{CDCl}_{3}\right) 23.0$, $23.3,25.0,30.2,95.3,116.1,117.6,124.4,125.5,126.1,128.9,129.0,131.0,139.1,140.0,146.1$; IR $\left(\mathrm{CDCl}_{3}\right) 3396,3054,2927,1578 \mathrm{~cm}^{-1}$; HRMS m/z 349.0331 (calcd for $\mathrm{C}_{16} \mathrm{H}_{16} \mathrm{IN}, 349.0328$ ).

$N$-Allyl-3-iodoaniline. This compound was prepared according to the reported procedure: ${ }^{5}{ }^{1} \mathrm{H}$ NMR $\left(\mathrm{CDCl}_{3}\right) \delta 3.74(\mathrm{~d}, J=5.1 \mathrm{~Hz}, 2 \mathrm{H}), 3.81(\mathrm{~s}, 1 \mathrm{H}), 5.19-5.33(\mathrm{~m}, 2 \mathrm{H}), 5.87-5.99(\mathrm{~m}, 1 \mathrm{H}), 6.57(\mathrm{dd}, J=8.1$, $2.4 \mathrm{~Hz}, 1 \mathrm{H}), 6.89(\mathrm{t}, J=8.1 \mathrm{~Hz}, 1 \mathrm{H}), 6.97(\mathrm{t}, J=1.6 \mathrm{~Hz}, 1 \mathrm{H}), 7.05(\mathrm{~d}, J=7.8 \mathrm{~Hz}, 1 \mathrm{H}) ;{ }^{13} \mathrm{C} \mathrm{NMR}$ $\left(\mathrm{CDCl}_{3}\right) 46.5,95.6,112.5,116.9,121.7,126.6,130.9,135.0,149.5 ; \mathrm{IR}\left(\mathrm{CDCl}_{3}\right) 3417,3076,2847,1590$ $\mathrm{cm}^{-1}$; HRMS m/z 258.9862 (calcd for $\mathrm{C}_{9} \mathrm{H}_{10} \mathrm{NI}, 258.9858$ ).

3-(3-Iodophenylamino)cyclohex-2-enone. 3-Iodoaniline (2 mmol) and cyclohexane-1,3-dione (2 mmol) were dissolved in $10 \mathrm{~mL}$ toluene and then the mixture was heated at $100{ }^{\circ} \mathrm{C}$ in the presence of 8 mmol anhydrous $\mathrm{MgSO}_{4}$ and a catalytic amount of $\mathrm{TsOH}$. After $12 \mathrm{~h}$, the reaction mixture was filtered, and the toluene was removed from the filtrate. The residue obtained was purified by flash chromatography to afford a 90\% yield of the imine product: ${ }^{1} \mathrm{H} \mathrm{NMR}\left(\mathrm{CDCl}_{3}\right) \delta 1.96-2.02(\mathrm{~m}, 2 \mathrm{H}), 2.32$ $(\mathrm{t}, J=6.2 \mathrm{~Hz}, 2 \mathrm{H}), 2.50(\mathrm{t}, J=6.2 \mathrm{~Hz}, 2 \mathrm{H}), 5.51(\mathrm{~s}, 1 \mathrm{H}), 7.00(\mathrm{t}, J=8.0 \mathrm{~Hz}, 1 \mathrm{H}), 7.10(\mathrm{dd}, J=8.0,0.9$ $\mathrm{Hz}, 1 \mathrm{H}), 7.40-7.48(\mathrm{~m}, 3 \mathrm{H}) ;{ }^{13} \mathrm{C} \mathrm{NMR}\left(\mathrm{CDCl}_{3}\right)$ 22.0, 29.8, 36.7, 123.3, 130.9, 132.8, 134.6, 139.8, 
162.8, 198.9; IR $\left(\mathrm{CDCl}_{3}\right)$ 3247, 3056, 2945, $1566 \mathrm{~cm}^{-1}$; HRMS m/z 312.9968 (calcd for $\mathrm{C}_{12} \mathrm{H}_{12} \mathrm{INO}$, 312.9964).

1-Iodo-3-phenoxybenzene. This compound was prepared by the reported procedure: ${ }^{6} \mathrm{H}_{\mathrm{NMR}}\left(\mathrm{CDCl}_{3}\right)$

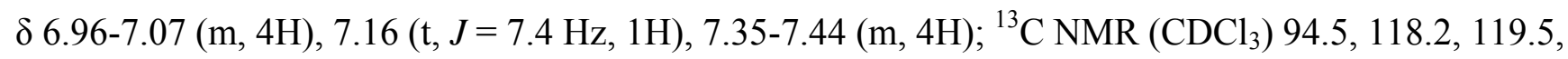
124.2, 127.8, 130.2, 131.3, 132.4, 156.6, 158.3; IR $\left(\mathrm{CDCl}_{3}\right) 3075,2965,1582 \mathrm{~cm}^{-1} ; \mathrm{HRMS} \mathrm{m} / \mathrm{z}$ 295.9702 (calcd for $\mathrm{C}_{12} \mathrm{H}_{9} \mathrm{IO}, 295.9698$ ).

1-Iodo-3,5-diphenoxybenzene. This compound was prepared by the strategy shown below: ${ }^{1} \mathrm{H}$ NMR $\left(\mathrm{CDCl}_{3}\right) \delta 6.63(\mathrm{t}, J=2.2 \mathrm{~Hz}, 1 \mathrm{H}), 7.02-7.04(\mathrm{~m}, 6 \mathrm{H}), 7.13-7.17(\mathrm{~m}, 2 \mathrm{H}), 7.35-7.39(\mathrm{~m}, 4 \mathrm{H}) ;{ }^{13} \mathrm{C} \mathrm{NMR}$ $\left(\mathrm{CDCl}_{3}\right)$ 94.1, 108.7, 119.8, 122.0, 124.5, 130.2, 156.1, 159.4; IR $\left(\mathrm{CDCl}_{3}\right) 3073,3039,1575 \mathrm{~cm}^{-1}$; HRMS m/z 387.9964 (calcd for $\mathrm{C}_{18} \mathrm{H}_{13} \mathrm{IO}_{2}, 387.9960$ ).<smiles>CC#CC(C)CC(C)C(C)C</smiles>
(i) (a) $\mathrm{NaNO}_{2}, \mathrm{HCl}$ (b) $\mathrm{KI}$; (ii) $\mathrm{BBr}_{3}$; (iii) $6 \mathrm{CsF}, 2.2$ 2-(trimethylsilyl)phenyl trifluoromethanesulfonate, $\mathrm{MeCN}$.

1-Iodo-3-methoxy-5-phenoxybenzene. This compound was prepared by the strategy shown below: ${ }^{7}$ ${ }^{1} \mathrm{H} \operatorname{NMR}\left(\mathrm{CDCl}_{3}\right) \delta 3.76(\mathrm{~s}, 3 \mathrm{H}), 6.57(\mathrm{t}, J=2.2 \mathrm{~Hz}, 1 \mathrm{H}), 6.97(\mathrm{t}, J=1.5 \mathrm{~Hz}, 1 \mathrm{H}), 7.03-7.09(\mathrm{~m}, 3 \mathrm{H})$, $7.18(\mathrm{t}, J=7.3 \mathrm{~Hz}, 1 \mathrm{H}), 7.36-7.42(\mathrm{~m}, 2 \mathrm{H}) ;{ }^{13} \mathrm{C} \mathrm{NMR}\left(\mathrm{CDCl}_{3}\right)$ 55.9, 94.4, 104.9, 118.3, 119.8, 120.2, 124.3, 130.2, 156.4, 159.3, 161.5; IR $\left(\mathrm{CDCl}_{3}\right) 3074,2960,1586 \mathrm{~cm}^{-1} ; \mathrm{HRMS} \mathrm{m} / \mathrm{z} 325.9808$ (calcd for $\left.\mathrm{C}_{13} \mathrm{H}_{11} \mathrm{IO}_{2}, 325.9804\right)$.<smiles>CC#CC1CC(C)CC1(C)CC(C)C</smiles>
(i) NaSMe, DMA, $140{ }^{\circ} \mathrm{C}$; (ii) (a) $\mathrm{NaNO}_{2}, \mathrm{HCl}$; (b) KI; (iii) $4 \mathrm{CsF}, 1.1$ 2-(trimethylsilyl)phenyl trifluoromethanesulfonate, $\mathrm{MeCN}$.

Compound 29-d: ${ }^{1} \mathrm{H}$ NMR $\left(\mathrm{CDCl}_{3}\right) \delta 3.76(\mathrm{~s}, 3 \mathrm{H}), 6.52(\mathrm{~s}, 0.08 \mathrm{H}), 6.92(\mathrm{~s}, 0.11 \mathrm{H}), 6.99-7.04(\mathrm{~m}, 2 \mathrm{H})$, $7.15(\mathrm{t}, J=7.32 \mathrm{~Hz}, 1 \mathrm{H})$, 7.34-7.39 (m, 2H). 
III. Experimental Procedures. The aryl halide $(0.25 \mathrm{mmol})$, alkyne $(0.25 \mathrm{mmol}), \mathrm{Pd}(\mathrm{OAc})_{2}(2.8 \mathrm{mg}$, $0.0125 \mathrm{mmol})$, bis(diphenylphosphino)methane (dppm) $(4.8 \mathrm{mg}, 0.0125 \mathrm{mmol})$ and $\mathrm{CsO}_{2} \mathrm{CCMe}_{3}(\mathrm{CsPiv})$ $(0.117 \mathrm{~g}, 0.5 \mathrm{mmol})$ in $4 \mathrm{~mL}$ of DMF were stirred under Ar at $100{ }^{\circ} \mathrm{C}$ for $6 \mathrm{~h}$. The reaction mixture was allowed to cool to room temperature, diluted with diethyl ether $(25 \mathrm{~mL})$ and washed with $5 \% \mathrm{Na}_{2} \mathrm{CO}_{3}$ $(25 \mathrm{~mL})$. The aqueous layer was re-extracted with diethyl ether $(25 \mathrm{~mL})$ twice. The organic layers were combined, dried $\left(\mathrm{MgSO}_{4}\right)$, filtered, and the solvent was removed under reduced pressure. The residue was purified by flash chromatography on silica gel.

For the products reported in entries $1,5,9,13,15$ and 16 of Table 2; entry 1 in Table 3; and entries 2-4, 8, and 9 in Table 3, GC-mass spectral analysis shows two regioisomers, which cannot be separated by flash chromatography. The ratio of these isomers was determined by ${ }^{1} \mathrm{H}$ NMR spectroscopy. (E)-4-(1-Phenylbut-1-enyl)-9H-carbazole (1a). ${ }^{1} \mathrm{H}$ NMR $\left(\mathrm{CDCl}_{3}\right) \delta 1.11$ (t, J=7.4 Hz, 3H), 2.94 (q, $J=7.4 \mathrm{~Hz}, 2 \mathrm{H}), 6.74(\mathrm{~s}, 1 \mathrm{H}), 7.11-7.20(\mathrm{~m}, 2 \mathrm{H}), 7.35-7.53(\mathrm{~m}, 9 \mathrm{H}), 8.05(\mathrm{~s}, 1 \mathrm{H}), 8.20(\mathrm{~d}, J=7.9 \mathrm{~Hz}$, $1 \mathrm{H}) ;{ }^{13} \mathrm{C} \mathrm{NMR}\left(\mathrm{CDCl}_{3}\right)$ 13.4, 25.9, 109.4, 110.7, 119.6, 120.2, 120.9, 123.0, 123.3, 125.7, 125.8, 126.9, 128.7, 128.9, 129.0, 138.2, 139.9, 139.9, 140.1, 144.6; IR $\left(\mathrm{CDCl}_{3}\right) 3471,3056,2968,2934,1599 \mathrm{~cm}^{-1}$; HRMS m/z 297.1522 (calcd for $\mathrm{C}_{22} \mathrm{H}_{19} \mathrm{~N}, 297.1518$ ).

(E)-4-(4,4-Dimethylpent-2-en-2-yl)-9H-carbazole (2). ${ }^{1} \mathrm{H} \mathrm{NMR}\left(\mathrm{CDCl}_{3}\right) \delta 1.34(\mathrm{~s}, 9 \mathrm{H}), 2.28(\mathrm{~d}, J=$ $1.3 \mathrm{~Hz}, 3 \mathrm{H}), 5.67(\mathrm{~d}, J=1.4 \mathrm{~Hz}, 1 \mathrm{H}), 6.96(\mathrm{dd}, J=6.9,1.2 \mathrm{~Hz}, 1 \mathrm{H}), 7.19-7.42(\mathrm{~m}, 5 \mathrm{H}), 8.06(\mathrm{~s}, 1 \mathrm{H})$, $8.14(\mathrm{~d}, J=7.8 \mathrm{~Hz}, 1 \mathrm{H}) ;{ }^{13} \mathrm{C} \mathrm{NMR}\left(\mathrm{CDCl}_{3}\right)$ 19.2, 31.2, 33.1, 108.8, 110.6, 119.4, 119.7, 120.2, 123.0, 123.2, 125.6, 125.9, 134.8, 139.6, 139.8, 139.9, 143.3; IR $\left(\mathrm{CDCl}_{3}\right) 3473,2960,2867,1600 \mathrm{~cm}^{-1}$; HRMS $\mathrm{m} / \mathrm{z} 263.1679$ (calcd for $\mathrm{C}_{19} \mathrm{H}_{21} \mathrm{~N}, 263.1674$ ).

4-[1-(2,2-Dimethylpropylidene)pentyl]-9H-carbazole (4a). ${ }^{1} \mathrm{H} \mathrm{NMR}\left(\mathrm{CDCl}_{3}\right) \delta 0.81(\mathrm{t}, J=7.2 \mathrm{~Hz}$, $3 \mathrm{H}), 1.22-1.40(\mathrm{~m}, 13 \mathrm{H}), 2.71-2.78(\mathrm{~m}, 2 \mathrm{H}), 5.62(\mathrm{~s}, 1 \mathrm{H}), 6.97(\mathrm{dd}, J=7.1,1.1 \mathrm{~Hz}, 1 \mathrm{H}), 7.19-7.42(\mathrm{~m}$, 5H), $8.01(\mathrm{~s}, 1 \mathrm{H}), 8.20(\mathrm{~d}, J=8.1 \mathrm{~Hz}, 1 \mathrm{H}) ;{ }^{13} \mathrm{C} \mathrm{NMR}\left(\mathrm{CDCl}_{3}\right)$ 14.2, 23.4, 31.4, 31.7, 32.4, 33.2, 108.7, $110.6,119.3,120.5,120.7,123.2,123.4,125.5,139.3,139.8,139.9,140.1,141.9 ; \mathrm{IR}\left(\mathrm{CDCl}_{3}\right) 3410$ 2957, 2866, $1599 \mathrm{~cm}^{-1}$; HRMS m/z 305.2148 (calcd for $\mathrm{C}_{22} \mathrm{H}_{27} \mathrm{~N}, 305.2144$ ). 
(E)-4-(1-Phenylprop-1-enyl)-9H-carbazole (5a). ${ }^{1} \mathrm{H} \mathrm{NMR}\left(\mathrm{CDCl}_{3}\right) \delta 2.47(\mathrm{~s}, 3 \mathrm{H}), 6.78(\mathrm{~s}, 1 \mathrm{H}), 7.12-$ $7.20(\mathrm{~m}, 2 \mathrm{H}), 7.33-7.54(\mathrm{~m}, 9 \mathrm{H}), 8.10(\mathrm{~s}, 1 \mathrm{H}), 8.14(\mathrm{~d}, J=7.8 \mathrm{~Hz}, 1 \mathrm{H}) ;{ }^{13} \mathrm{C} \mathrm{NMR}\left(\mathrm{CDCl}_{3}\right)$ 19.9, 109.4, $110.7,119.4,119.6,120.2,122.9,123.1,125.8,125.9,126.8,128.6,129.3,129.4,138.3(2 \mathrm{C}), 139.9$ 140.1, 141.4; IR $\left(\mathrm{CDCl}_{3}\right) 3471,3060,3026,1601,1456 \mathrm{~cm}^{-1} ; \mathrm{HRMS} \mathrm{m} / \mathrm{z} 283.1367$ (calcd for $\mathrm{C}_{18} \mathrm{H}_{19} \mathrm{~N}$, $283.1361)$.

(E)-4-(1,2-Diphenylvinyl)-9H-carbazole (6). ${ }^{1} \mathrm{H} \mathrm{NMR}\left(\mathrm{CDCl}_{3}\right) \delta$ 7.03-7.14 (m, 3H), 7.23-7.42 (m, 14H), $8.03(\mathrm{~s}, 1 \mathrm{H}), 8.37(\mathrm{~d}, J=8.1 \mathrm{~Hz}, 1 \mathrm{H}) ;{ }^{13} \mathrm{C} \mathrm{NMR}\left(\mathrm{CDCl}_{3}\right)$ 109.8, 110.8, 119.6, 121.3, 121.7, 123.0, $123.39,125.7,125.9,127.3,127.7,128.5,128.7,129.8,130.2,131.1,137.6,140.0,140.3,140.4,140.7$, 141.3; IR $\left(\mathrm{CDCl}_{3}\right) 3414,3054,1599,1455 \mathrm{~cm}^{-1}$; HRMS m/z 345.1522 (calcd for $\mathrm{C}_{26} \mathrm{H}_{19} \mathrm{~N}, 345.1518$ ). (E)-Ethyl 4-[1-(9H-carbazol-4-yl)but-1-enyl]benzoate (7a). ${ }^{1} \mathrm{H} \mathrm{NMR}\left(\mathrm{CDCl}_{3}\right) \delta 1.90(\mathrm{t}, J=7.5 \mathrm{~Hz}$, $3 \mathrm{H}), 1.44(\mathrm{t}, J=7.2 \mathrm{~Hz}, 3 \mathrm{H}), 1.91$ (q, $J=7.5 \mathrm{~Hz}, 2 \mathrm{H}), 4.43$ (q, $J=7.2 \mathrm{~Hz}, 2 \mathrm{H}), 6.73(\mathrm{~s}, 1 \mathrm{H}), 7.10$ (dd, $J$ $=6.7,1.7 \mathrm{~Hz}, 1 \mathrm{H}), 7.15(\mathrm{td}, J=6.8,1.5 \mathrm{~Hz}, 1 \mathrm{H}), 7.37-7.46(\mathrm{~m}, 4 \mathrm{H}), 7.53(\mathrm{~d}, J=8.1 \mathrm{~Hz}, 2 \mathrm{H}), 8.10-8.20$ $(\mathrm{m}, 4 \mathrm{H}) ;{ }^{13} \mathrm{C} \mathrm{NMR}\left(\mathrm{CDCl}_{3}\right)$ 13.3, 14.6, 26.0, 6.19, 109.6, 110. 8, 119.6, 119.9, 120.8, 122.8, 123.1, $125.6,125.8,128.2,128.8,128.9,130.0,139.4,139.9,140.1,142.8,146.7,166.8 ; \mathrm{IR}\left(\mathrm{CDCl}_{3}\right) 3472$, 2968, 2873, $1710 \mathrm{~cm}^{-1}$; HRMS m/z 369.1736 (calcd for $\mathrm{C}_{25} \mathrm{H}_{23} \mathrm{NO}_{2}, 369.1729$ ).

(E)-4-[1-(2-Methoxyphenyl)but-1-enyl]-9H-carbazole (8a). ${ }^{1} \mathrm{H} \mathrm{NMR}\left(\mathrm{CDCl}_{3}\right) \delta 1.00(\mathrm{t}, J=7.5 \mathrm{~Hz}$, 3H), $2.80(\mathrm{q}, J=7.5 \mathrm{~Hz}, 2 \mathrm{H}), 3.84(\mathrm{~s}, 3 \mathrm{H}), 6.77(\mathrm{~s}, 1 \mathrm{H}), 6.95(\mathrm{~d}, J=8.0 \mathrm{~Hz}, 1 \mathrm{H}), 6.97-7.17(\mathrm{~m}, 3 \mathrm{H})$, 7.29-7.53 (m, 6H), $8.11(\mathrm{~s}, 1 \mathrm{H}), 8.36(\mathrm{~d}, J=7.8 \mathrm{~Hz}, 1 \mathrm{H}) ;{ }^{13} \mathrm{C} \mathrm{NMR}\left(\mathrm{CDCl}_{3}\right)$ 13.3, 26.1, 55.5, 109.1, $110.5,110.8,118.3,119.3,120.2,120.4,121.2,123.5,124.7,125.5,125.6,127.3,128.3,130.1,139.9$ 140.1, 144.0, 157.8; IR $\left(\mathrm{CDCl}_{3}\right)$ 3472, 2966, 2934, $1245 \mathrm{~cm}^{-1}$; HRMS m/z 327.1628 (calcd for $\left.\mathrm{C}_{23} \mathrm{H}_{21} \mathrm{NO}, 327.1623\right)$.

(E)-5-(1,2-Diphenylvinyl)-3-methyl-9H-carbazole (9). ${ }^{1} \mathrm{H} \mathrm{NMR}\left(\mathrm{CDCl}_{3}\right) \delta 2.36(\mathrm{~s}, 3 \mathrm{H}), 7.02-7.05$ (m, 2H), 7.20-7.40 (m, 14H), $7.95(\mathrm{~s}, 1 \mathrm{H}), 8.10(\mathrm{~s}, 1 \mathrm{H}) ;{ }^{13} \mathrm{C} \mathrm{NMR}\left(\mathrm{CDCl}_{3}\right)$ 21.8, 109.8, 110.4, 121.3, $121.6,123.1,123.5,125.5,127.2,127.2,127.6,128.5,128.5,128.6,129.7,130.3,131.1,137.8,138.2$ 140.2, 140.5, 140.7, 141.4; IR $\left(\mathrm{CDCl}_{3}\right) 3413,3053,3022,1599,1491 \mathrm{~cm}^{-1} ; \mathrm{HRMS} \mathrm{m} / \mathrm{z} 359.1678$ (calcd for $\mathrm{C}_{27} \mathrm{H}_{21} \mathrm{~N}, 359.1674$ ). 
(E)-5-(1,2-Diphenylvinyl)-3-methoxy-9H-carbazole (10). ${ }^{1} \mathrm{H} \mathrm{NMR}\left(\mathrm{CDCl}_{3}\right) \delta 3.58(\mathrm{~s}, 3 \mathrm{H})$, 7.02-7.07 $(\mathrm{m}, 3 \mathrm{H}), 7.22-7.42(\mathrm{~m}, 13 \mathrm{H}), 7.81(\mathrm{~d}, J=2.5 \mathrm{~Hz}, 1 \mathrm{H}), 7.98(\mathrm{~s}, 1 \mathrm{H}) ;{ }^{13} \mathrm{C} \mathrm{NMR}\left(\mathrm{CDCl}_{3}\right) 55.8,105.2$, $110.1,111.5,115.8,121.5,121.6,123.6,125.6,127.3,127.8,128.7,129.7,130.3,131.3,134.8,137.7$, 140.1, 140.5, 141.1 (2C), 153.5; IR $\left(\mathrm{CDCl}_{3}\right) 3415,3054,2949,1582,1478 \mathrm{~cm}^{-1} ; \mathrm{HRMS} \mathrm{m} / \mathrm{z} 375.1629$ (calcd for $\mathrm{C}_{27} \mathrm{H}_{21} \mathrm{NO}, 375.1623$ ).

5-(1-Benzylidenepropyl)-1-methoxy-9H-carbazole (11a). ${ }^{1} \mathrm{H} \mathrm{NMR}\left(\mathrm{CDCl}_{3}\right) \delta 1.08(\mathrm{t}, J=7.5 \mathrm{~Hz}, 3 \mathrm{H})$, $2.91(\mathrm{q}, J=7.5 \mathrm{~Hz}, 2 \mathrm{H}), 4.0(\mathrm{~s}, 3 \mathrm{H}), 6.7(\mathrm{~s}, 1 \mathrm{H}), 6.89(\mathrm{~d}, J=7.6 \mathrm{~Hz}, 1 \mathrm{H}), 7.06-7.10(\mathrm{~m}, 2 \mathrm{H}), 7.30-7.50$ $(\mathrm{m}, 7 \mathrm{H}), 7.77(\mathrm{~d}, J=8.0 \mathrm{~Hz}, 1 \mathrm{H}), 8.37(\mathrm{~s}, 1 \mathrm{H}) ;{ }^{13} \mathrm{C} \mathrm{NMR}\left(\mathrm{CDCl}_{3}\right)$ 13.4, 25.9, 55.8, 105.8, 109.7, 115.6, $119.7,120.0,124.2,125.5,126.8,128.6,128.9,129.0,130.3,138.2,139.8,139.9,144.5,145.8 ;$ IR $\left(\mathrm{CDCl}_{3}\right)$ 3421, 2964, 2932, $1598 \mathrm{~cm}^{-1}$; HRMS m/z 327.1625 (calcd for $\mathrm{C}_{23} \mathrm{H}_{21} \mathrm{NO}, 327.1623$ ).

Methyl 5-(1,2-diphenylvinyl)-9H-carbazole-3-carboxylate (12). ${ }^{1} \mathrm{H} \mathrm{NMR}\left(\mathrm{CDCl}_{3}\right) \delta 3.72(\mathrm{~s}, 3 \mathrm{H})$, $7.03(\mathrm{~s}, 1 \mathrm{H}), 7.09(\mathrm{t}, J=4.2 \mathrm{~Hz}, 1 \mathrm{H}), 7.20-7.42(\mathrm{~m}, 13 \mathrm{H}), 8.07(\mathrm{dd}, J=8.5,1.4 \mathrm{~Hz}, 1 \mathrm{H}), 8.41(\mathrm{~s}, 1 \mathrm{H})$, $9.00(\mathrm{~d}, J=1.4 \mathrm{~Hz}, 1 \mathrm{H}) ;{ }^{13} \mathrm{C} \mathrm{NMR}\left(\mathrm{CDCl}_{3}\right) 51.9,110.1,110.2,121.4,121.6,122.6,122.9,125.6,126.4$, $127.2,127.5,127.7,128.3,128.5,129.8,130.4,131.5,137.5,140.0,140.5,140.7,140.9,142.7,167.9$; IR $\left(\mathrm{CDCl}_{3}\right)$ 3323, 3021, 2947, $1691 \mathrm{~cm}^{-1} ; \mathrm{HRMS} \mathrm{m} / \mathrm{z} 403.1578$ (calcd for $\mathrm{C}_{28} \mathrm{H}_{21} \mathrm{NO}_{2}, 403.1572$ ) (E)-3-Chloro-5-(1-phenylbut-1-enyl)-9H-carbazole (13a). ${ }^{1} \mathrm{H} \mathrm{NMR}\left(\mathrm{CDCl}_{3}\right) \delta 1.09(\mathrm{t}, J=7.5 \mathrm{~Hz}$, 3H), $2.91(\mathrm{q}, J=7.5 \mathrm{~Hz}, 2 \mathrm{H}), 6.73(\mathrm{~s}, 1 \mathrm{H}), 7.14(\mathrm{~d}, J=7.2 \mathrm{~Hz}, 1 \mathrm{H}), 7.29-7.52(\mathrm{~m}, 10 \mathrm{H}), 8.08(\mathrm{~s}, 1 \mathrm{H})$, $8.19(\mathrm{~d}, J=1.5 \mathrm{~Hz}, 1 \mathrm{H}) ;{ }^{13} \mathrm{C} \mathrm{NMR}\left(\mathrm{CDCl}_{3}\right)$ 13.4, 25.7, 109.6, 111.7, 120.5, 122.6, 124.5, 124.9, 125.9, 126.4, 127.1, 128.8, 129.2, 129.7, 137.9, 138.2, 140.0, 140.6, 144.0; IR $\left(\mathrm{CDCl}_{3}\right) 3471,2944,2833 \mathrm{~cm}^{-1}$; HRMS m/z 331.1132 (calcd for $\mathrm{C}_{22} \mathrm{H}_{18} \mathrm{NCl}, 331.1128$ ).

(E)-7-(1-Phenylbut-1-enyl)-11H-benzo[a]carbazole (14a). ${ }^{1} \mathrm{H} \mathrm{NMR}\left(\mathrm{CDCl}_{3}\right) \delta 1.10(\mathrm{t}, J=7.6 \mathrm{~Hz}$, 3H), $2.95(\mathrm{q}, J=7.6 \mathrm{~Hz}, 2 \mathrm{H}), 6.75(\mathrm{~s}, 1 \mathrm{H}), 7.16(\mathrm{dd}, J=7.3,1.2 \mathrm{~Hz}, 1 \mathrm{H}), 7.26-7.60(\mathrm{~m}, 10 \mathrm{H}), 7.98(\mathrm{~d}$, $J=7.5 \mathrm{~Hz}, 1 \mathrm{H}), 8.14(\mathrm{~d}, J=8.1 \mathrm{~Hz}, 1 \mathrm{H}), 8.23(\mathrm{~d}, J=8.1 \mathrm{~Hz}, 1 \mathrm{H}), 8.87(\mathrm{~s}, 1 \mathrm{H}) ;{ }^{13} \mathrm{C} \mathrm{NMR}\left(\mathrm{CDCl}_{3}\right)$ $13.3,26.1,109.8,118.5,120.1,120.6(2 \mathrm{C}), 121.1,121.8,121.9,124.7,125.5,125.7,126.9,128.7,129.0$ 129.1 (2C), 132.3, 135.3, 138.2, 139.1, 139.3, 144.5; IR $\left(\mathrm{CDCl}_{3}\right) 3472,3060,2969,1572 \mathrm{~cm}^{-1}$; HRMS $\mathrm{m} / \mathrm{z} 347.1682$ (calcd for $\mathrm{C}_{26} \mathrm{H}_{21} \mathrm{~N}, 347.1674$ ). 
7-(1,2-Diphenylvinyl)-2,3,4,11-tetrahydro-1H-benzo[a]carbazole (15). ${ }^{1} \mathrm{H} \mathrm{NMR}\left(\mathrm{CDCl}_{3}\right) \delta 1.91$ -

$2.03(\mathrm{~m}, 4 \mathrm{H}), 2.93(\mathrm{~m}, 4 \mathrm{H}), 6.86(\mathrm{~d}, J=8.2 \mathrm{~Hz}, 1 \mathrm{H}), 6.99(\mathrm{dd}, J=7.2,0.8 \mathrm{~Hz}, 1 \mathrm{H}), 7.07$ (s, 1H), 7.23-

$7.40(\mathrm{~m}, 12 \mathrm{H}), 8.01(\mathrm{~s}, 1 \mathrm{H}), 8.09(\mathrm{~d}, J=8.2 \mathrm{~Hz}, 1 \mathrm{H}) ;{ }^{13} \mathrm{C} \mathrm{NMR}\left(\mathrm{CDCl}_{3}\right)$ 23.1, 13.6, 24.6, 29.9, 109.8, $119.1,120.0,120.4,121.2,121.6,122.0,124.9,127.2,127.6,128.4,128.6,129.8,130.2,131.0,134.9$, 137.7, 139.1, 139.9, 140.2, 140.8, 141.3; IR $\left(\mathrm{CDCl}_{3}\right) 3434,3053,2929,1601 \mathrm{~cm}^{-1}$; $\mathrm{HRMS} \mathrm{m} / \mathrm{z}$ 399.1993 (calcd for $\mathrm{C}_{30} \mathrm{H}_{25} \mathrm{~N}, 399.1987$ ).

(E)-3-Methyl-4-(1-phenylbut-1-en-2-yl)-1H-indole (16a). ${ }^{1} \mathrm{H} \mathrm{NMR}\left(\mathrm{CDCl}_{3}\right) \delta 1.09$ (t, $\left.J=7.2 \mathrm{~Hz}, 3 \mathrm{H}\right)$, $2.36(\mathrm{~s}, 3 \mathrm{H}), 2.77$ (q, J = 7.2 Hz, 2H), $6.47(\mathrm{~s}, 1 \mathrm{H}), 6.96-6.99(\mathrm{~m}, 2 \mathrm{H}), 7.18(\mathrm{t}, J=7.8 \mathrm{~Hz}, 1 \mathrm{H}), 7.27-$ $7.32(\mathrm{~m}, 2 \mathrm{H}), 7.41(\mathrm{~d}, J=4.1 \mathrm{~Hz}, 4 \mathrm{H}), 7.93(\mathrm{~s}, 1 \mathrm{H}) ;{ }^{13} \mathrm{C} \mathrm{NMR}\left(\mathrm{CDCl}_{3}\right)$ 13.2, 13.4, 27.1, 110.0, 112.5, $119.7,121.7,122.9,125.6,126.6,128.5,128.9,129.0,137.3,137.9,138.4,144.4 ; \mathrm{IR}\left(\mathrm{CDCl}_{3}\right) 3418$, 3021, 2964, $1598 \mathrm{~cm}^{-1}$; HRMS m/z 261.1518 (calcd for $\mathrm{C}_{19} \mathrm{H}_{19} \mathrm{~N}, 261.1521$ ).

4-(1,2-Diphenylvinyl)-3-methyl-1H-indole (17). ${ }^{1} \mathrm{H} \mathrm{NMR}\left(\mathrm{CDCl}_{3}\right) \delta 2.24(\mathrm{~d}, J=0.8 \mathrm{~Hz}, 3 \mathrm{H}), 6.68(\mathrm{~s}$, 1H), $6.89(\mathrm{dd}, J=7.2,0.8 \mathrm{~Hz}, 1 \mathrm{H}), 6.99(\mathrm{~d}, J=1.0 \mathrm{~Hz}, 1 \mathrm{H}), 7.11-7.32(\mathrm{~m}, 12 \mathrm{H}), 7.99(\mathrm{~s}, 1 \mathrm{H}) ;{ }^{13} \mathrm{C} \mathrm{NMR}$ $\left(\mathrm{CDCl}_{3}\right)$ 13.7, 110.4, 112.8, 121.7, 121.8, 123.2, 126.8, 127.3, 128.3 (2C), 128.4, 129.6, 130.5, 137.6, 137.9, 138.1, 141.3, 141.4; IR $\left(\mathrm{CDCl}_{3}\right)$ 3422, 3053, 3021, $1695 \mathrm{~cm}^{-1}$; HRMS m/z 309.1522 (calcd for $\left.\mathrm{C}_{23} \mathrm{H}_{19} \mathrm{~N}, 309.1518\right)$.

3-Methyl-4-(1-methyl-2-phenylvinyl)-1H-indole (18a). ${ }^{1} \mathrm{H} \mathrm{NMR}\left(\mathrm{CDCl}_{3}\right) \delta 2.34-2.35$ (m, 6H), 6.51 $(\mathrm{d}, J=1.0 \mathrm{~Hz}, 1 \mathrm{H}), 6.96-6.99(\mathrm{~m}, 2 \mathrm{H}), 7.18(\mathrm{t}, J=8.0 \mathrm{~Hz}, 1 \mathrm{H}), 7.26-7.31(\mathrm{~m}, 3 \mathrm{H}), 7.39-7.43(\mathrm{~m}, 4 \mathrm{H})$, $7.96(\mathrm{~s}, 1 \mathrm{H}) ;{ }^{13} \mathrm{C} \mathrm{NMR}\left(\mathrm{CDCl}_{3}\right)$ 12.8, 21.8, 110.1, 112.4, 119.1, 121.9, 122.8, 126.5, 128.4, 129.2, 129.4, 137.3, 138.5 (2C), 139.8; IR $\left(\mathrm{CDCl}_{3}\right)$ 3416, 3051, 2919, $1597 \mathrm{~cm}^{-1}$; HRMS m/z 247.1365 (calcd for $\left.\mathrm{C}_{18} \mathrm{H}_{17} \mathrm{~N}, 247.1361\right)$.

5-(1,2-Diphenylvinyl)-1,2,3,9-tetrahydro-carbazol-4-one (19). ${ }^{1} \mathrm{H} \mathrm{NMR}\left(\mathrm{CDCl}_{3}\right) \delta 2.05-2.12(\mathrm{~m}, 2 \mathrm{H})$, $2.48(\mathrm{t}, J=6.1 \mathrm{~Hz}, 2 \mathrm{H}), 2.80(\mathrm{t}, J=6.2 \mathrm{~Hz}, 2 \mathrm{H}), 6.51(\mathrm{~s}, 1 \mathrm{H}), 7.02-7.34(\mathrm{~m}, 13 \mathrm{H}), 9.72(\mathrm{~s}, 1 \mathrm{H})$; NMR $\left(\mathrm{CDCl}_{3}\right)$ 23.4, 23.8, 38.8, 110.8, 113.9, 123.9, 124.4, 125.4, 126.2, 126.8, 127.2, 127.3, 127.9, 128.0, 129.5, 134.0, 137.0, 138.5, 143.4, 144.2, 191.9; IR $\left(\mathrm{CDCl}_{3}\right) 3168,3052,2952,1621 \mathrm{~cm}^{-1} ; \mathrm{HRMS} \mathrm{m} / \mathrm{z}$ 363.1631 (calcd for $\mathrm{C}_{26} \mathrm{H}_{21} \mathrm{NO}, 363.1623$ ). 
1-(1-Benzylidenepropyl)-3-phenoxydibenzofuran (21a). ${ }^{1} \mathrm{H} \mathrm{NMR}\left(\mathrm{CDCl}_{3}\right) \delta 1.11(\mathrm{t}, J=7.5 \mathrm{~Hz}, 3 \mathrm{H})$, $7.87(\mathrm{q}, J=7.5 \mathrm{~Hz}, 2 \mathrm{H}), 6.76(\mathrm{~s}, 1 \mathrm{H}), 7.05(\mathrm{~d}, J=2.2 \mathrm{~Hz}, 1 \mathrm{H}), 7.04-7.58(\mathrm{~m}, 14 \mathrm{H}), 8.01(\mathrm{dd}, J=7.7$, $0.6 \mathrm{~Hz}, 1 \mathrm{H}) ;{ }^{13} \mathrm{C} \mathrm{NMR}\left(\mathrm{CDCl}_{3}\right)$ 13.3, 25.6, 100.9, 111.7, 114.5, 117.8, 119.3, 122.2, 123.0, 123.9, 124.2, $126.4,127.3,128.4,128.8,129.8,130.2,137.6,140.9,142.5,156.9,157.4,157.5 ; \mathrm{IR}\left(\mathrm{CDCl}_{3}\right) 3023$, 2966, $1628 \mathrm{~cm}^{-1}$; HRMS m/z 390.1624 (calcd for $\mathrm{C}_{28} \mathrm{H}_{22} \mathrm{O}_{2}, 390.1620$ ).

1-(1-Benzylidenepropyl)-3-methoxydibenzofuran (22a). ${ }^{1} \mathrm{H} \mathrm{NMR}\left(\mathrm{CDCl}_{3}\right) \delta 1.11(\mathrm{t}, J=7.6 \mathrm{~Hz}, 3 \mathrm{H})$, $2.88(\mathrm{q}, J=7.6 \mathrm{~Hz}, 2 \mathrm{H}), 3.94(\mathrm{~s}, 3 \mathrm{H}), 6.73(\mathrm{~s}, 1 \mathrm{H}), 6.87(\mathrm{~s}, 1 \mathrm{H}), 7.05(\mathrm{~s}, 1 \mathrm{H}), 7.21-7.57(\mathrm{~m}, 8 \mathrm{H}), 7.82(\mathrm{~d}$, $J=7.2 \mathrm{~Hz}, 1 \mathrm{H}) ;{ }^{13} \mathrm{C} \mathrm{NMR}\left(\mathrm{CDCl}_{3}\right)$ 13.2, 25.6, 56.0, 95.2, 111.0, 111.5, 115.5, 121.9, 122.8, 124.6, 125.7, 127.2, 128.7, 129.5, 137.7, 140.6, 142.9, 156.7, 158.0, 159.7; IR $\left(\mathrm{CDCl}_{3}\right) 3056,3022,2964$, $1627 \mathrm{~cm}^{-1}$; HRMS m/z 328.1468 (calcd for $\mathrm{C}_{23} \mathrm{H}_{20} \mathrm{O}_{2}, 328.1463$ ).

3-Methoxy-1-(1-methyl-2-phenylvinyl)dibenzofuran (23a). ${ }^{1} \mathrm{H} \mathrm{NMR}\left(\mathrm{CDCl}_{3}\right) \delta 2.45(\mathrm{~d}, \mathrm{~J}=1.3 \mathrm{~Hz}$, 3H), $3.9(\mathrm{~s}, 3 \mathrm{H}), 6.81(\mathrm{~s}, 1 \mathrm{H}), 6.88(\mathrm{~d}, J=2.2 \mathrm{~Hz}, 1 \mathrm{H}), 7.07(\mathrm{~d}, J=2.2 \mathrm{~Hz}, 1 \mathrm{H}), 7.25-7.57(\mathrm{~m}, 8 \mathrm{H}), 7.93$ $(\mathrm{d}, J=7.2 \mathrm{~Hz}, 1 \mathrm{H}) ;{ }^{13} \mathrm{C} \mathrm{NMR}\left(\mathrm{CDCl}_{3}\right) 19.6,56.0,95.2,110.6,111.5,114.7,121.9,122.8,124.4,125.8$, $127.1,128.7,129.3,130.2,130.2,136.5,137.8,142.2,156.7,158.0,159.9 ; \mathrm{IR}\left(\mathrm{CDCl}_{3}\right) 3054,2938$ 2835, $1627 \mathrm{~cm}^{-1}$; HRMS m/z 314.1311 (calcd for $\mathrm{C}_{22} \mathrm{H}_{18} \mathrm{O}_{2}, 314.1307$ ).

3-Methoxy-1-(1,3,3-trimethylbut-1-enyl)dibenzofuran (24a). ${ }^{1} \mathrm{H} \mathrm{NMR}\left(\mathrm{CDCl}_{3}\right) \delta 1.31$ (s, 9H), 2.23 $(\mathrm{d}, J=1.0 \mathrm{~Hz}, 1 \mathrm{H}), 3.90(\mathrm{~s}, 1 \mathrm{H}), 5.68(\mathrm{~d}, J=1.0 \mathrm{~Hz}, 1 \mathrm{H}), 6.69(\mathrm{~d}, J=1.7 \mathrm{~Hz}, 1 \mathrm{H}), 6.97(\mathrm{~d}, J=1.7 \mathrm{~Hz}$ 1H), 7.25-7.38 (m, 3H), $7.51(\mathrm{~d}, J=6.1 \mathrm{~Hz}, 1 \mathrm{H}), 7.88(\mathrm{~d}, J=6.0 \mathrm{~Hz}, 1 \mathrm{H}) ;{ }^{13} \mathrm{C} \mathrm{NMR}\left(\mathrm{CDCl}_{3}\right) 18.9$, $31.2,56.0,94.5,110.7,111.4,121.8,122.6,124.5,125.5,130.1,133.2,140.5,143.8,156.5,157.8$, 159.8; IR $\left(\mathrm{CDCl}_{3}\right)$ 2956, 2865, $1628 \mathrm{~cm}^{-1}$; HRMS m/z 294.1624 (calcd for $\mathrm{C}_{20} \mathrm{H}_{22} \mathrm{O}_{2}, 294.1620$ ). 3-Methoxy-1-(1-propylpent-1-enyl)dibenzofuran (25). ${ }^{1} \mathrm{H} \mathrm{NMR}\left(\mathrm{CDCl}_{3}\right) \delta 0.89(\mathrm{t}, J=7.3 \mathrm{~Hz}, 3 \mathrm{H})$, $1.02(\mathrm{t}, J=7.4 \mathrm{~Hz}, 3 \mathrm{H}), 1.32-1.45(\mathrm{~m}, 2 \mathrm{H}), 1.48-1.60(\mathrm{~m}, 2 \mathrm{H}), 2.31(\mathrm{q}, J=7.3 \mathrm{~Hz}, 2 \mathrm{H}), 2.58(\mathrm{t}, J=7.4$ $\mathrm{Hz}, 2 \mathrm{H}), 3.90(\mathrm{~s}, 3 \mathrm{H}), 5.67(\mathrm{t}, J=7.2 \mathrm{~Hz}, 1 \mathrm{H}), 6.71(\mathrm{~d}, J=2.2 \mathrm{~Hz}, 1 \mathrm{H}), 6.99(\mathrm{~d}, J=2.2 \mathrm{~Hz}, 1 \mathrm{H}), 7.22-$ $7.38(\mathrm{~m}, 2 \mathrm{H}), 7.52(\mathrm{~d}, J=8.2 \mathrm{~Hz}, 1 \mathrm{H}), 7.88(\mathrm{~d}, J=7.8 \mathrm{~Hz}, 1 \mathrm{H}) ;{ }^{13} \mathrm{C} \mathrm{NMR}\left(\mathrm{CDCl}_{3}\right)$ 14.3, 14.4, 22.0, $23.2,30.6,33.6,55.9,94.5,111.3,111.4,115.2,121.9,122.6,124.7,125.5,130.9,138.8,141.4,156.6$, 157.8, 159.6; IR $\left(\mathrm{CDCl}_{3}\right)$ 2957, 2930, $2869 \mathrm{~cm}^{-1}$; HRMS m/z 308.1781 (calcd for $\mathrm{C}_{21} \mathrm{H}_{24} \mathrm{O}_{2}, 308.1776$ ). 
(E)-1-(1,2-Diphenylvinyl)-3-methoxydibenzofuran (26). ${ }^{1} \mathrm{H}$ NMR $\left(\mathrm{CDCl}_{3}\right) \delta 3.86(\mathrm{~s}, 3 \mathrm{H}), 6.72(\mathrm{~s}$, 1H), 7.06-7.37 (m, 14H), $7.54(\mathrm{~d}, J=8.1 \mathrm{~Hz}, 1 \mathrm{H}), 7.92(\mathrm{~d}, J=8.1 \mathrm{~Hz}, 1 \mathrm{H}) ;{ }^{13} \mathrm{C} \mathrm{NMR}\left(\mathrm{CDCl}_{3}\right) 56.0$, $95.7,111.5,112.8,115.8,122.0,122.8,124.5,125.9,127.5,127.9,128.5,128.8,129.8,130.3,131.6$, 137.1, 140.0, 140.1, 140.9, 156.8, 158.2, 159.6; IR $\left(\mathrm{CDCl}_{3}\right) 3054,3022,2958,1629 \mathrm{~cm}^{-1} ; \mathrm{HRMS} \mathrm{m} / \mathrm{z}$ 376.1470 (calcd for $\mathrm{C}_{27} \mathrm{H}_{20} \mathrm{O}_{2}, 376.1463$ ).

(E)-1-(2-Deutero-1,2-diphenylvinyl)-2,4-dideutero-3-methoxydibenzofuran (26-d). This compound contains $70 \%$ deuterium in the vinylic position: ${ }^{1} \mathrm{H}$ NMR $\left(\mathrm{CDCl}_{3}\right) \delta 3.86(\mathrm{~s}, 3 \mathrm{H}), 6.72(\mathrm{~s}, 0.30 \mathrm{H}), 7.09$ $7.37(\mathrm{~m}, 12 \mathrm{H}), 7.54(\mathrm{~d}, J=8.1 \mathrm{~Hz}, 1 \mathrm{H}), 7.92(\mathrm{~d}, J=8.1 \mathrm{~Hz}, 1 \mathrm{H})$. Compound 26-d obtained from the reaction conducted in the presence of 10 equiv of $\mathrm{D}_{2} \mathrm{O}:{ }^{1} \mathrm{H} \mathrm{NMR}\left(\mathrm{CDCl}_{3}\right) \delta 3.86(\mathrm{~s}, 3 \mathrm{H}), 6.72(\mathrm{~s}, 0.13 \mathrm{H})$, 7.37-7.37 (m, 12H), $7.54(\mathrm{~d}, J=8.1 \mathrm{~Hz}, 1 \mathrm{H}), 7.92(\mathrm{~d}, J=8.1 \mathrm{~Hz}, 1 \mathrm{H})$.

Ethyl 4-[2-(3-methoxydibenzofuran-1-yl)but-1-enyl]benzoate (27a). ${ }^{1} \mathrm{H} \mathrm{NMR}\left(\mathrm{CDCl}_{3}\right) \delta 1.09(\mathrm{t}, J=$ $7.4 \mathrm{~Hz}, 3 \mathrm{H}), 1.43(\mathrm{t}, J=7.1 \mathrm{~Hz}, 3 \mathrm{H}), 2.86(\mathrm{q}, J=7.4 \mathrm{~Hz}, 2 \mathrm{H}), 3.93(\mathrm{~s}, 3 \mathrm{H}), 4.42(\mathrm{q}, J=7.1 \mathrm{~Hz}, 2 \mathrm{H})$, $6.72(\mathrm{~s}, 1 \mathrm{H}), 6.83(\mathrm{~d}, J=2.2 \mathrm{~Hz}, 1 \mathrm{H}), 7.06(\mathrm{~d}, J=2.1 \mathrm{~Hz}, 1 \mathrm{H}), 7.22(\mathrm{t}, J=7.7 \mathrm{~Hz}, 1 \mathrm{H}), 7.33-7.55(\mathrm{~m}$, $4 \mathrm{H}), 7.87(\mathrm{~d}, J=7.7 \mathrm{~Hz}, 1 \mathrm{H}), 8.12(\mathrm{~d}, J=8.3 \mathrm{~Hz}, 2 \mathrm{H}) ;{ }^{13} \mathrm{C} \mathrm{NMR}\left(\mathrm{CDCl}_{3}\right)$ 13.2, 14.6, 25.7, 56.0, 61.2, $95.4,111.0,111.6,115.3,121.7,122.8,124.4,125.8,128.8,128.8,129.1,129.9,140.0,142.2,145.0$, 156.7, 158.0, 159.7, 166.7; IR $\left(\mathrm{CDCl}_{3}\right)$ 2969, 2935, 2873, $1716 \mathrm{~cm}^{-1}$; HRMS m/z 400.1679 (calcd for $\left.\mathrm{C}_{26} \mathrm{H}_{24} \mathrm{O}_{4}, 400.1675\right)$.

3-Methoxy-1-[1-(2-methoxybenzylidene)propyl]dibenzofuran (28a). ${ }^{1} \mathrm{H} \mathrm{NMR}\left(\mathrm{CDCl}_{3}\right) \delta 1.01(\mathrm{t}, J=$ $7.6 \mathrm{~Hz}, 3 \mathrm{H}), 2.77$ (q, $7.5 \mathrm{~Hz}, 2 \mathrm{H}), 3.84$ (s, 3H), 3.93 (s, 3H), 6.79 (s, 1H), 6.87 (d, J = 2.2 Hz, 1H), 6.95 $(\mathrm{d}, J=8.2 \mathrm{~Hz}, 1 \mathrm{H}), 7.03-7.08(\mathrm{~m}, 2 \mathrm{H}), 7.19-7.25(\mathrm{~m}, 1 \mathrm{H}), 7.30-7.38(\mathrm{~m}, 2 \mathrm{H}), 7.47-7.54(\mathrm{~m}, 2 \mathrm{H}), 8.14$ $(\mathrm{d}, J=7.2 \mathrm{~Hz}, 1 \mathrm{H}) ;{ }^{13} \mathrm{C} \mathrm{NMR}\left(\mathrm{CDCl}_{3}\right) 13.3,25.8,55.5,56.0,95.0,110.8,110.9,111.3,115.7,120.5$, $122.4,122.6,124.7,125.6,126.8,128.6,130.0,140.5,142.3,156.6,157.7,157.9,159.6 ; \mathrm{IR}\left(\mathrm{CDCl}_{3}\right)$ 2962, 2933, 2834, $1627 \mathrm{~cm}^{-1}$; HRMS m/z 358.1573 (calcd for $\mathrm{C}_{24} \mathrm{H}_{22} \mathrm{O}_{3}, 358.1569$ ). 


\section{References}

(1) Campo, M. A.; Larock, R. C. Org. Lett. 2000, 2, 3675.

(2) (a) Sonogashira, K. In Metal-Catalyzed Cross-Coupling Reactions; Diederich, F., Stang, P. J., Eds.; Wiley-VCH: Weinheim, Germany, 1998; Chapter 5, pp 203-229. (b) Sonogashira, K.; Tohda, Y.; Hagihara, N. Tetrahedron Lett. 1975, 4467.

(3) Liu, Z.; Larock, R. C. Org. Lett. 2003, 5, 4673.

(4) Driver, M. S.; Hartwig, J. F. J. Am. Chem. Soc. 1996, 118, 7217.

(5) Caddick, S.; Kofie, W. Tetrahedron Lett. 2002, 43, 9347.

(6) Liu, Z.; Larock, R. C. Org. Lett. 2004, 6, 99.

(7) For the preparation of 3-hydroxy-5-methoxyaniline, see: Wendt, M. D.; Rockway, T. W; Geyer, A.; McClellan, W.; Weitzberg, M.; Zhao, X.; Mantei, R.; Nienaber, V. L.; Stewart, K.; Klinghofer, V.; Giranda, V. J. Med. Chem. 2004, 47, 303. 
IV. Characterization Data for Selected Compounds.

2 Jffon

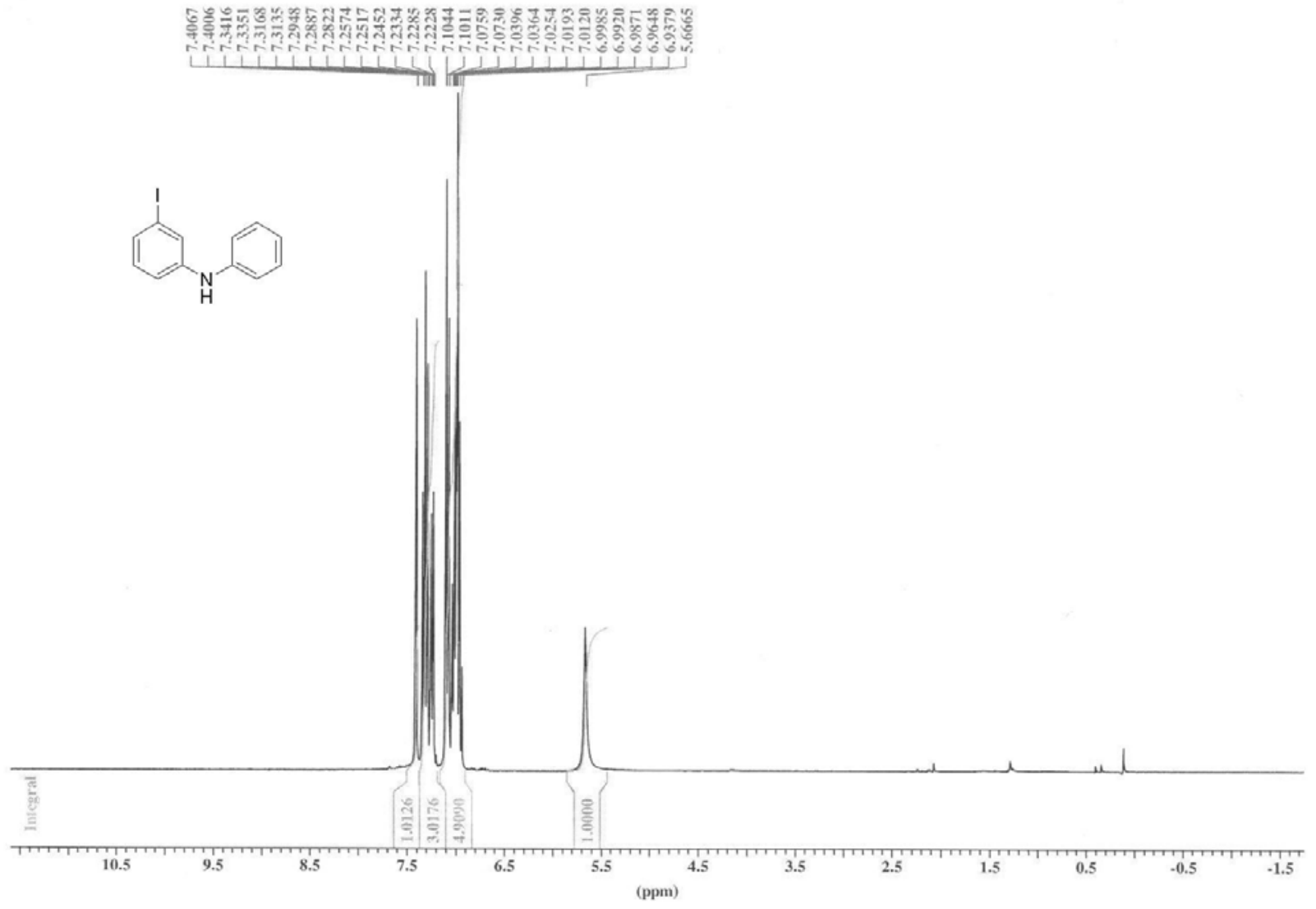




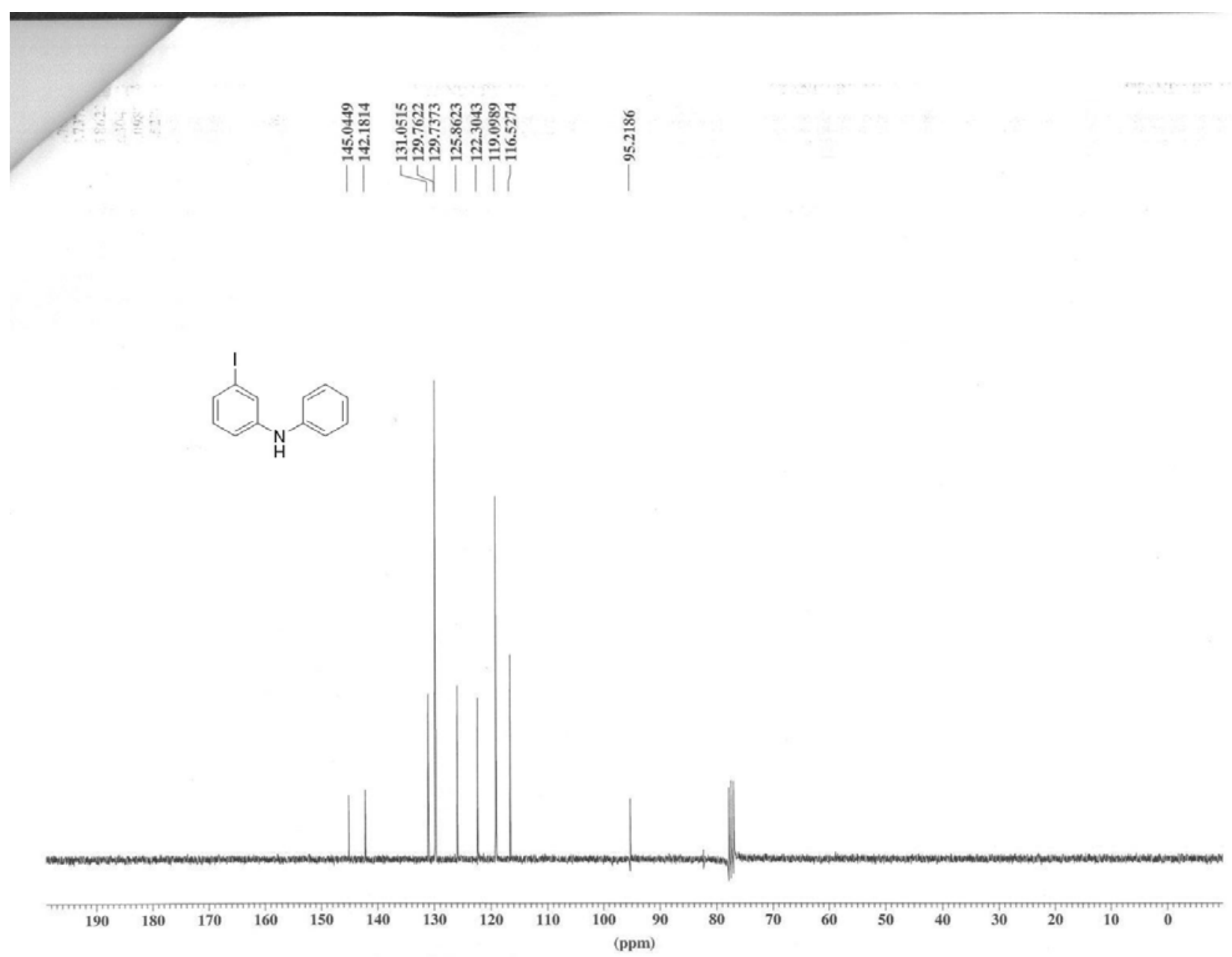



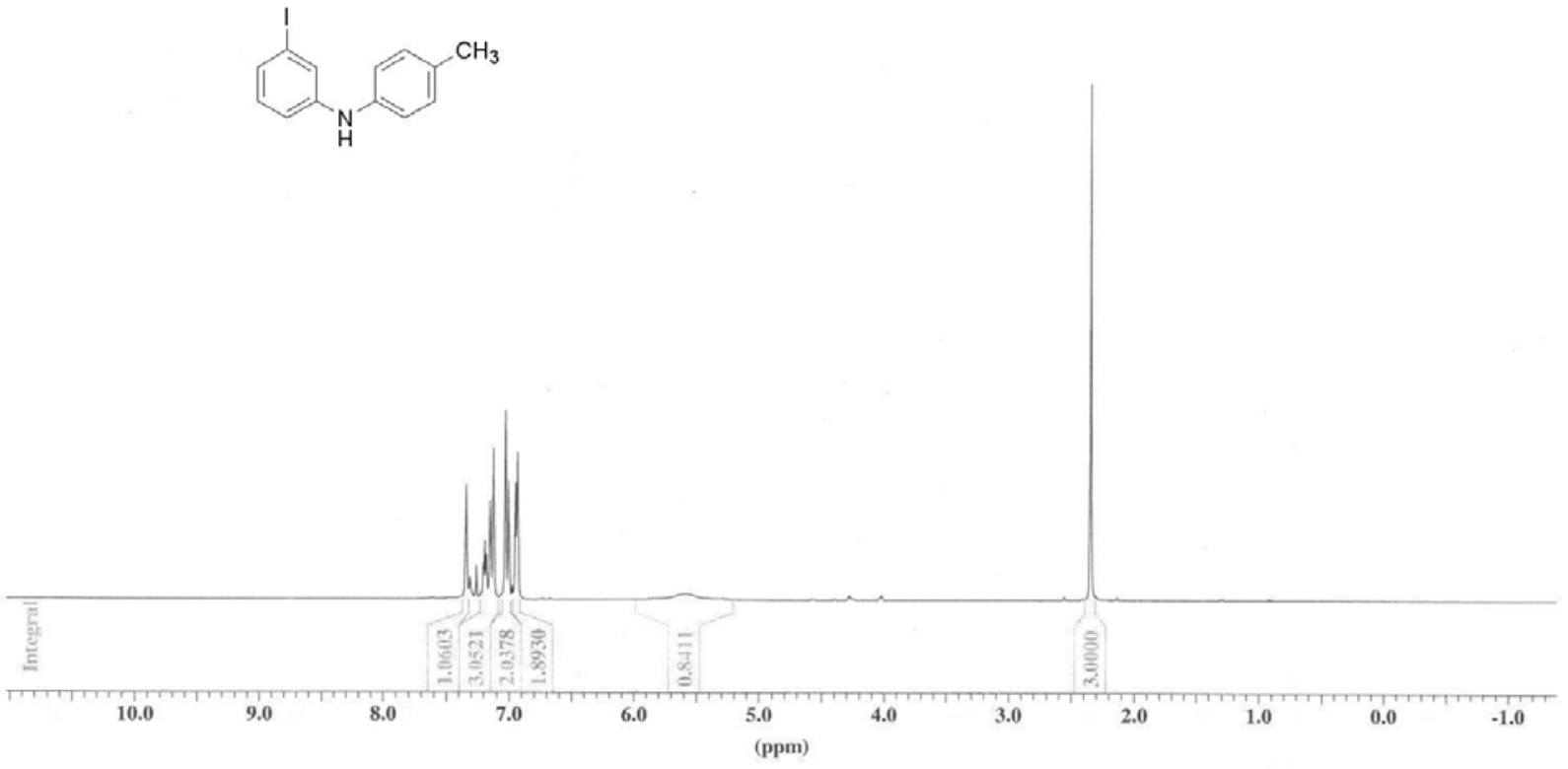

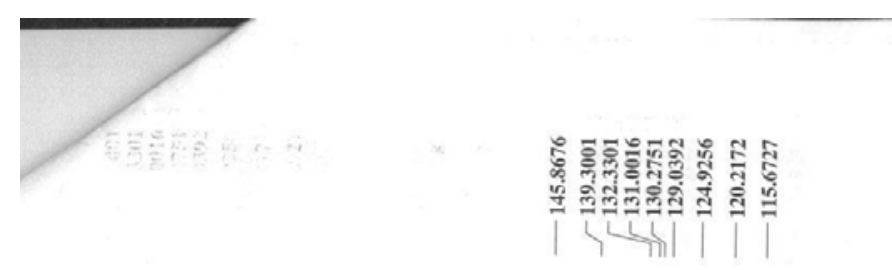

ำ

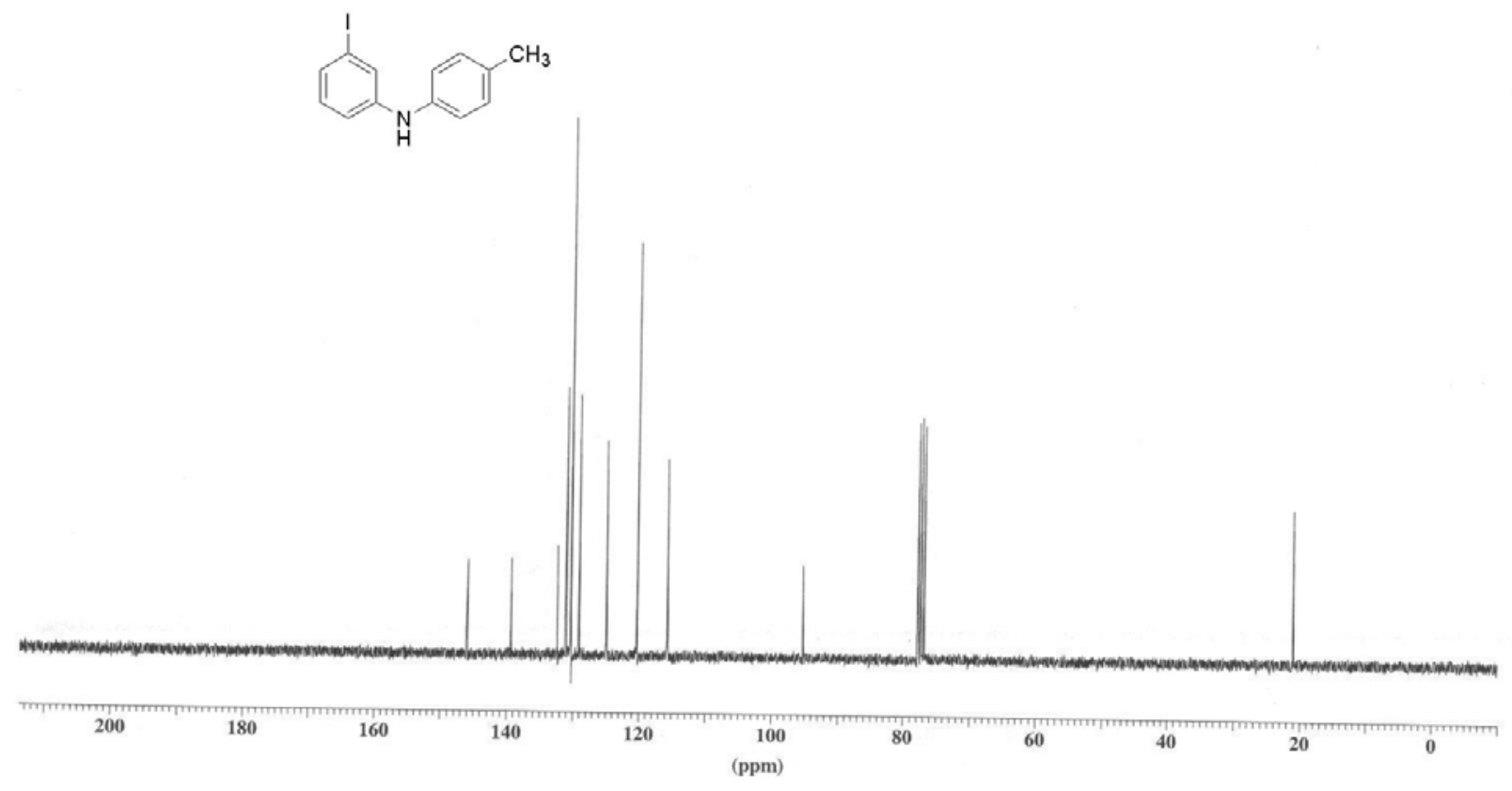


$2 J 447$ A
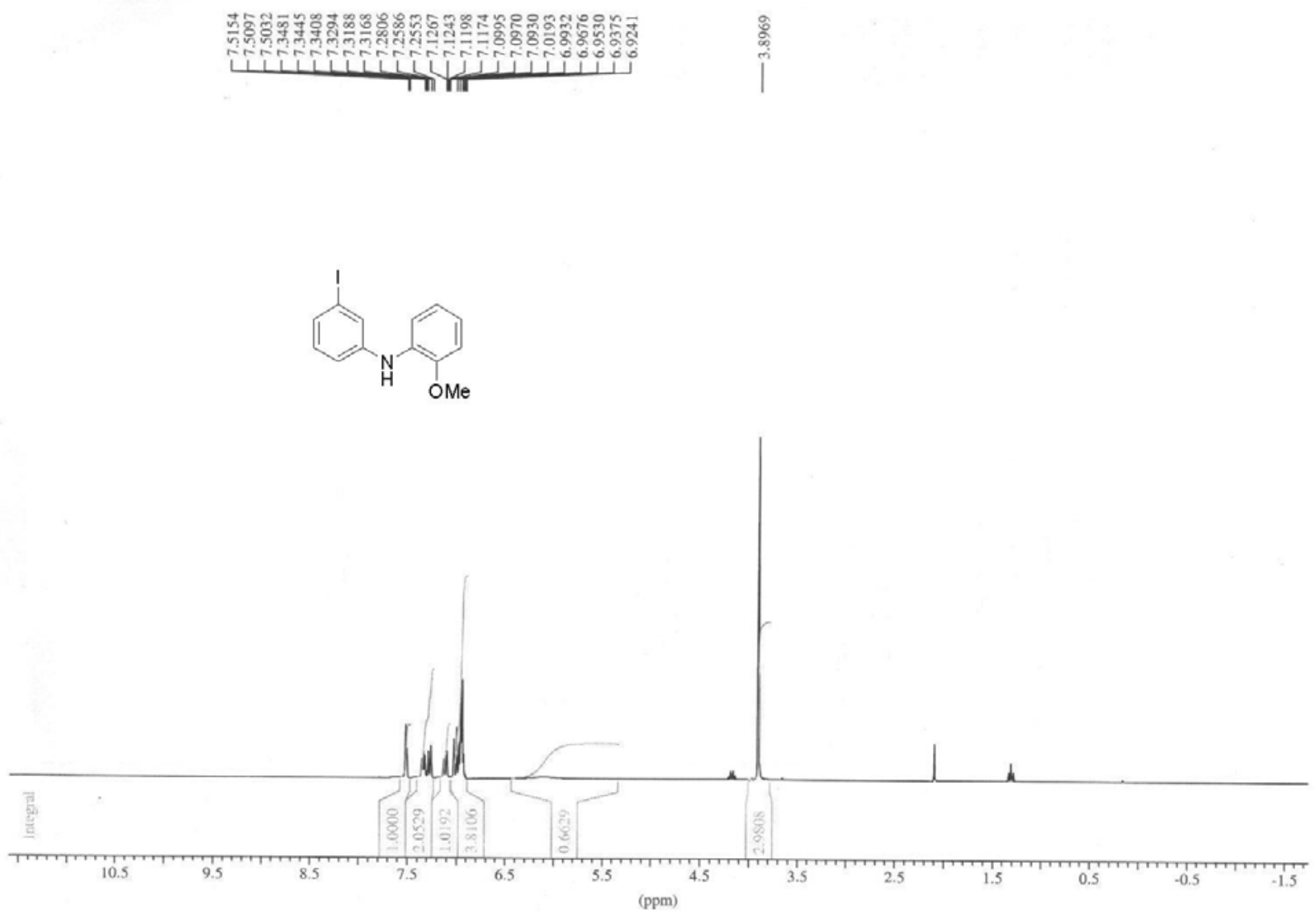


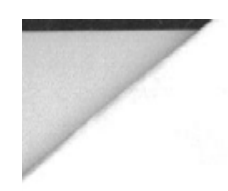

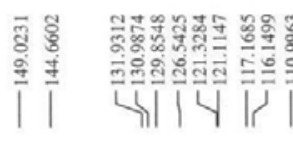
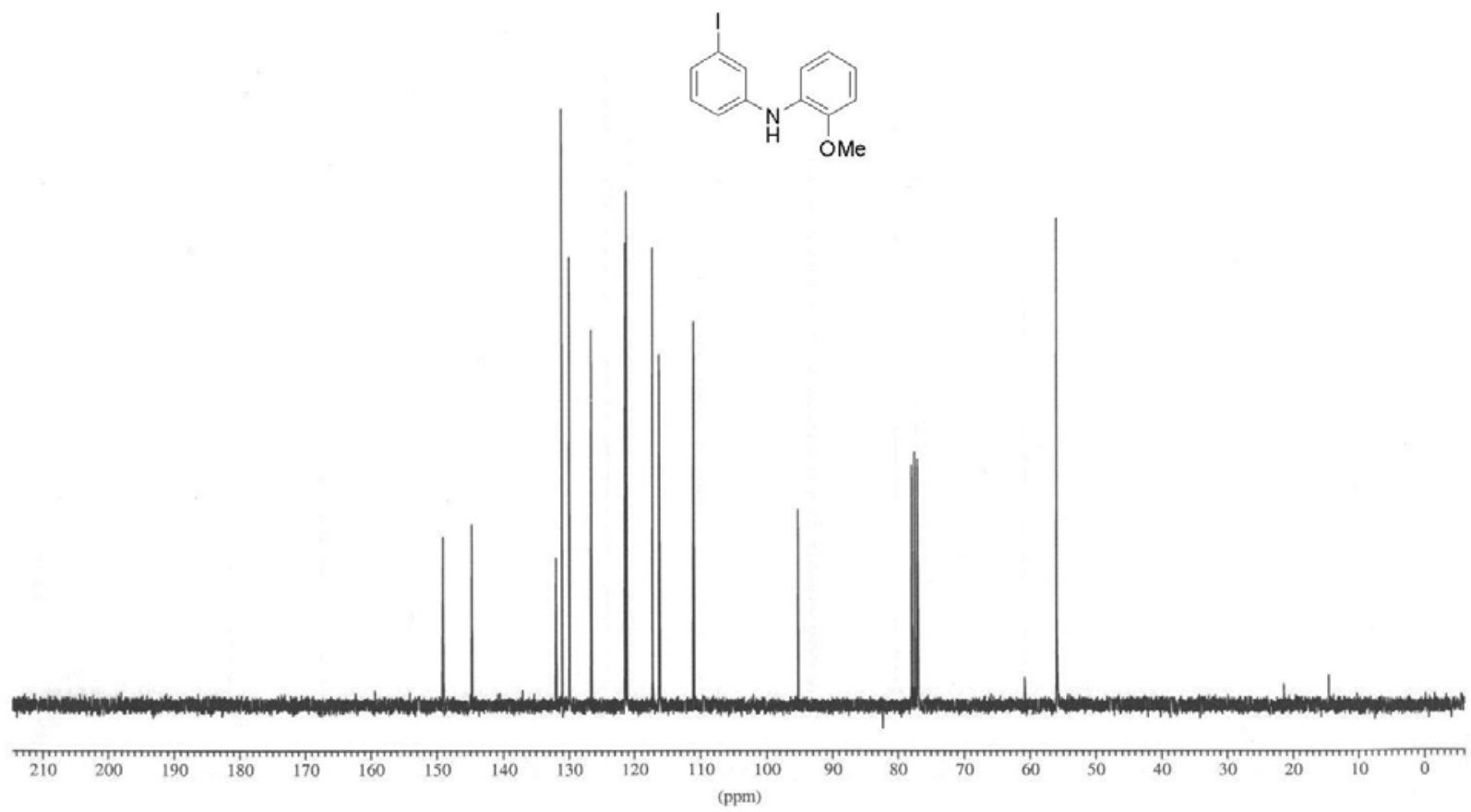
25438

STANDARD IH OBSERVE
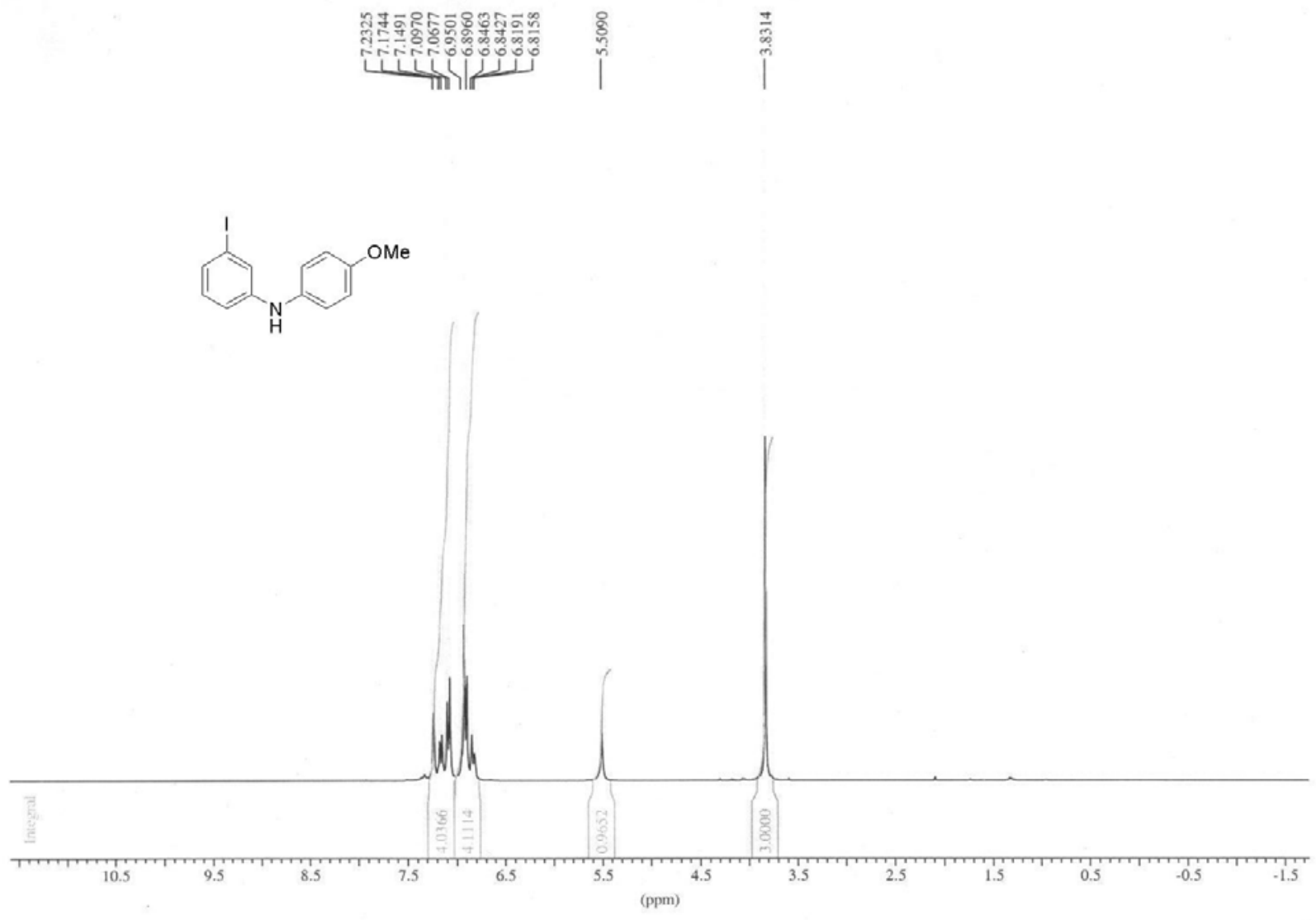


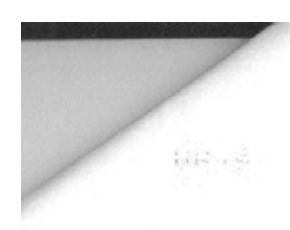

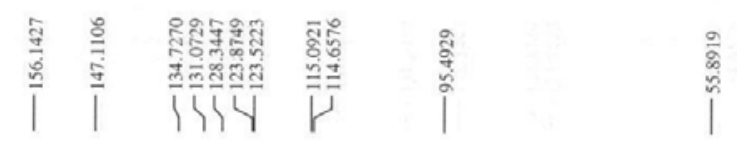

$\mathrm{H}_{\mathrm{H}}$

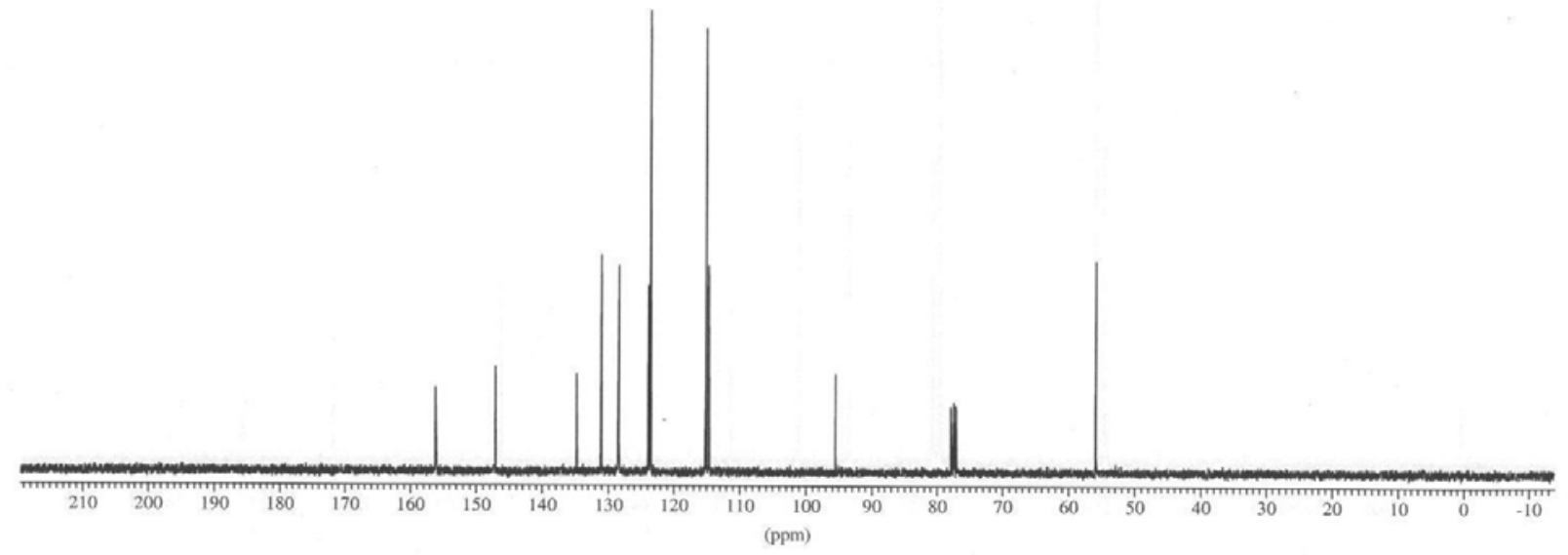


STANDARD 1 H OBSERVE
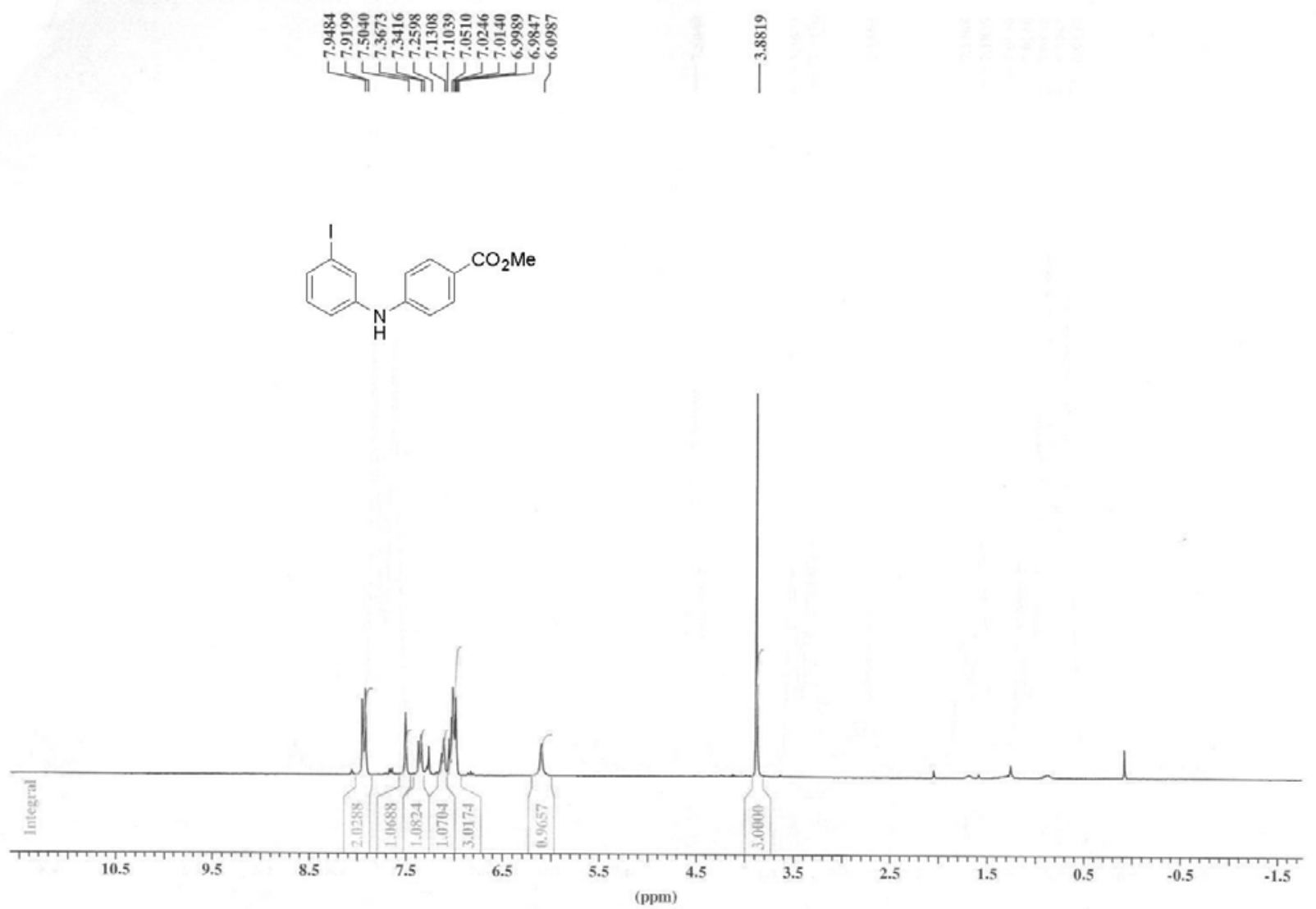

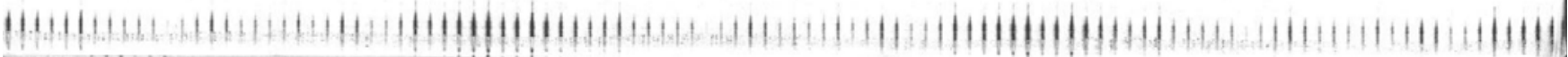




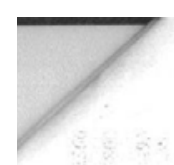

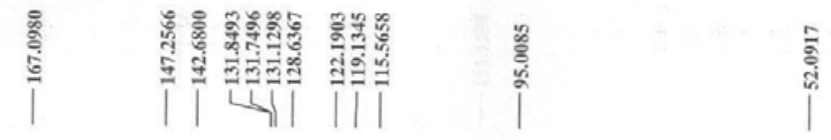<smiles>CC(=O)c1ccc(Nc2cccc(I)c2)cc1</smiles>

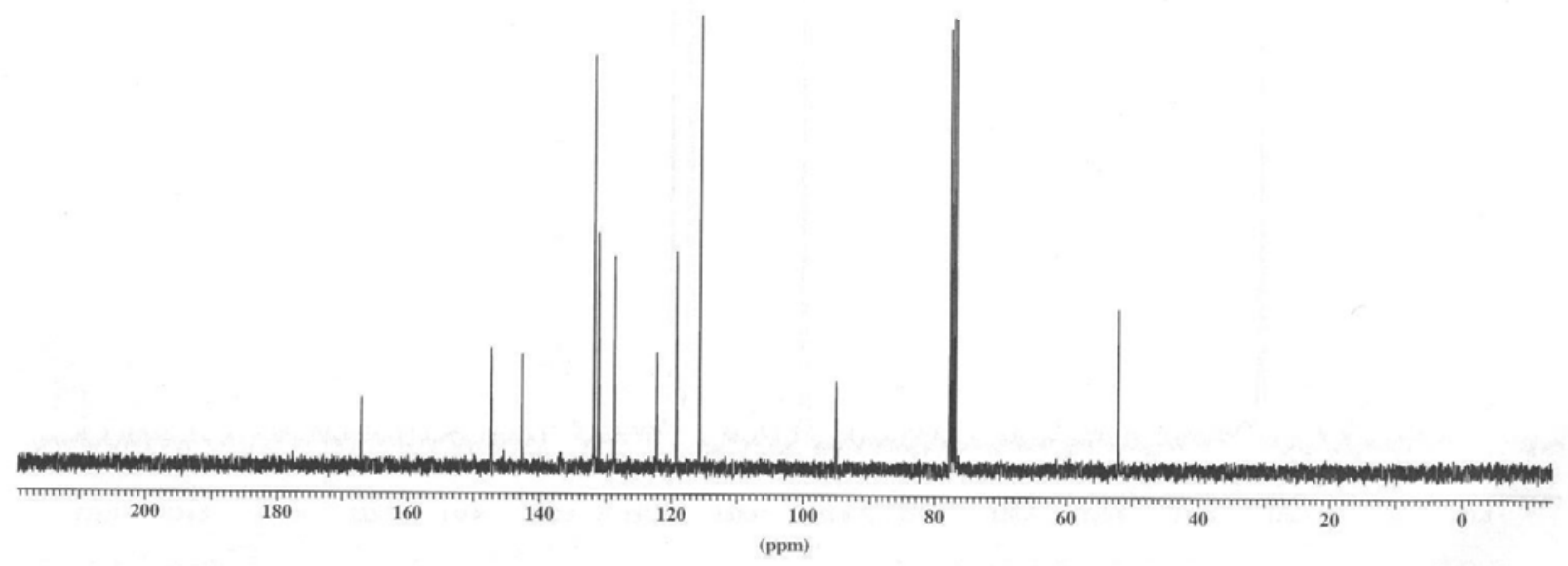

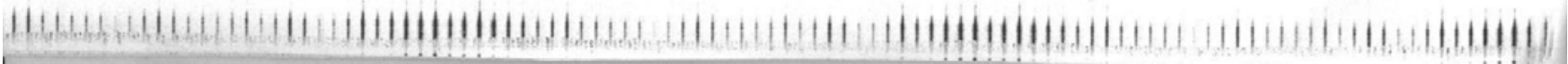


2于 $\mathrm{A}$ युस

ST ANDARD 1 H OBSERVE
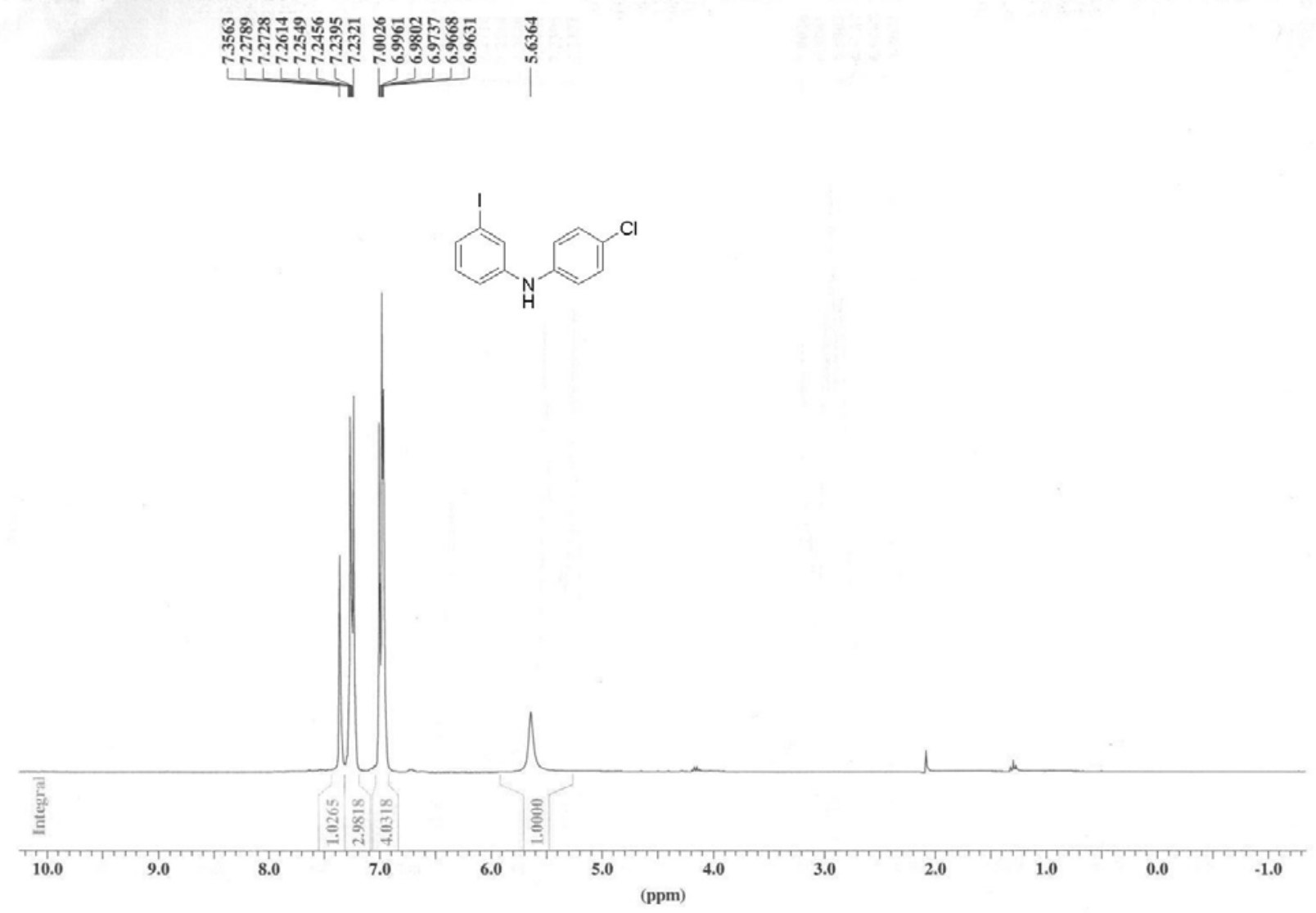


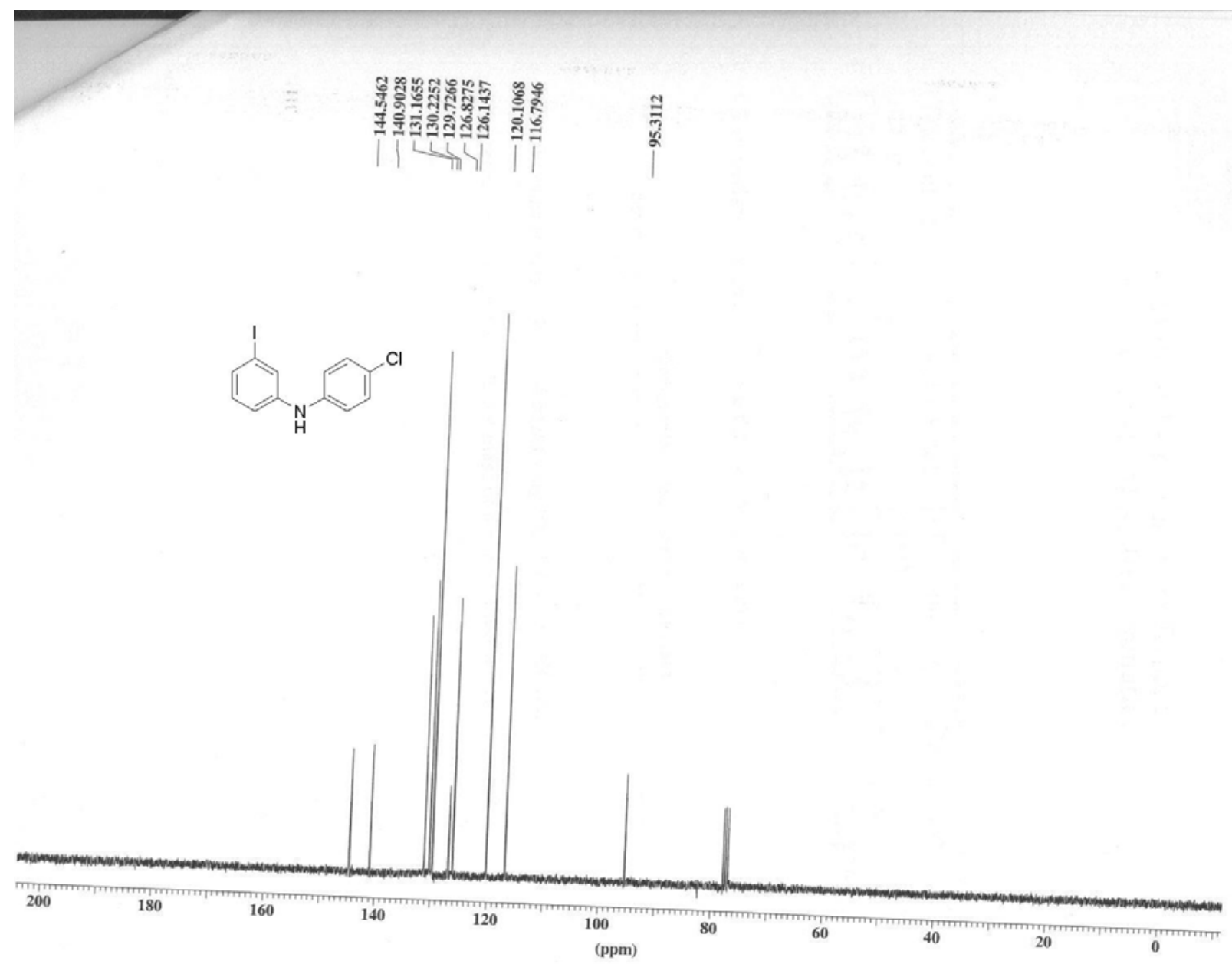



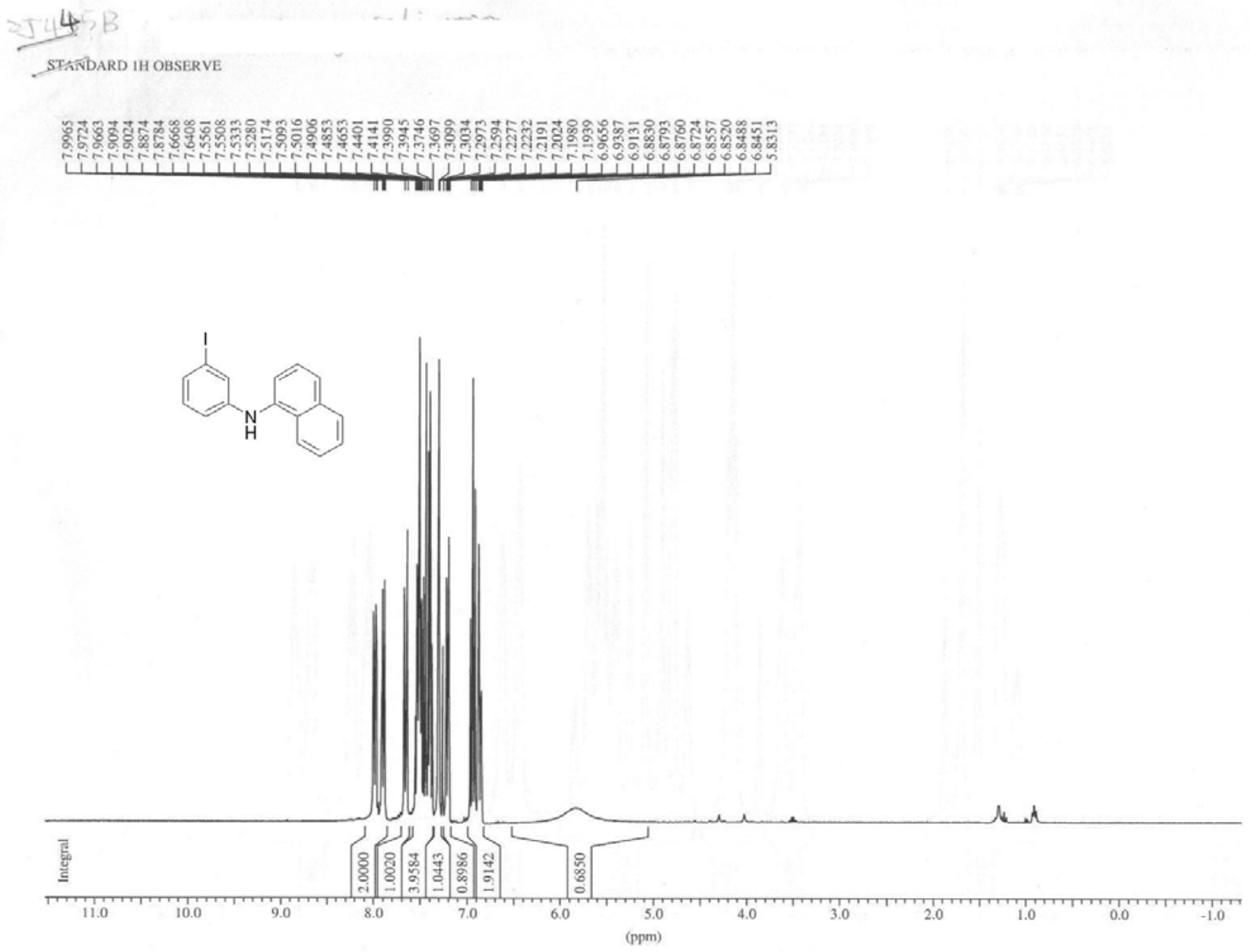

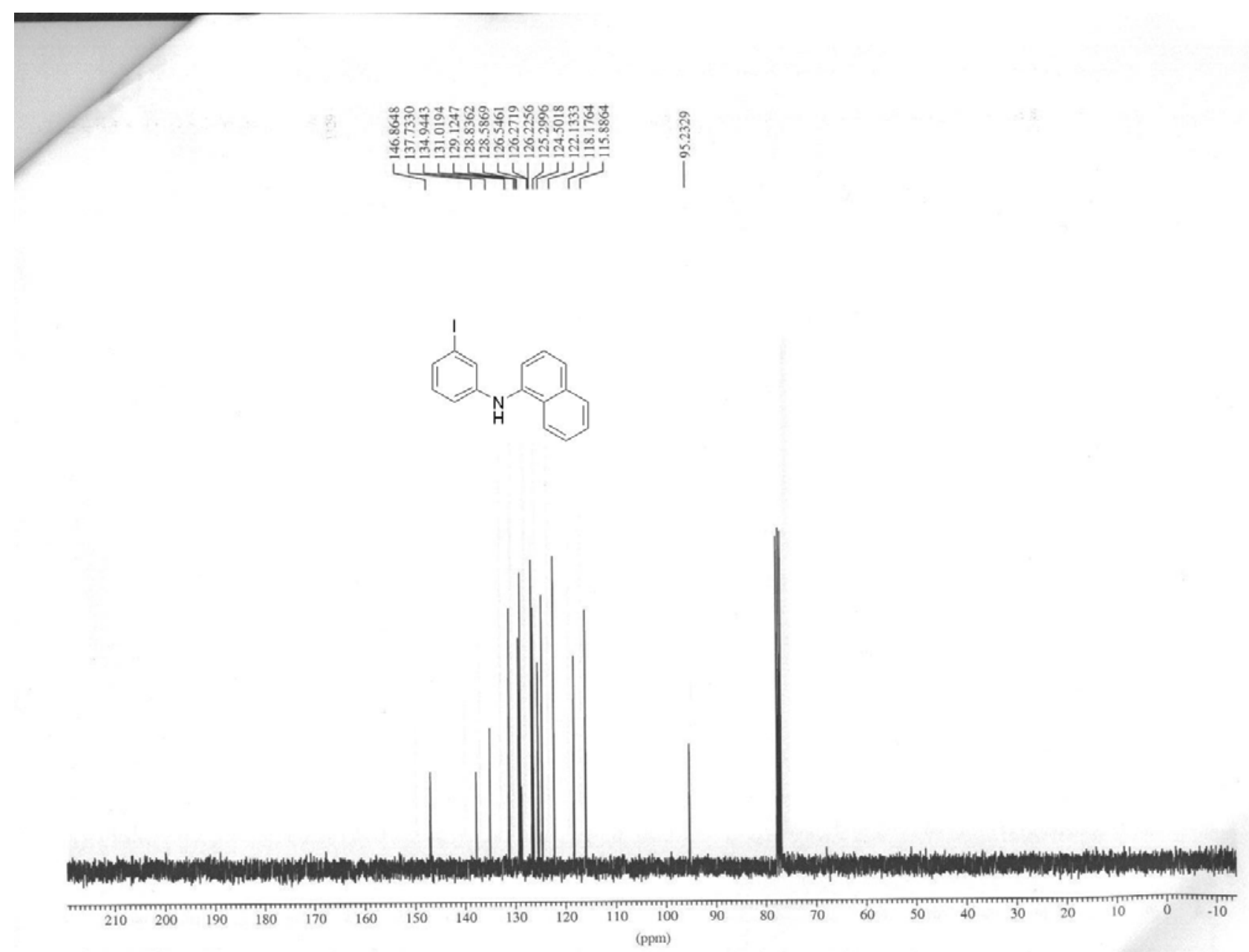

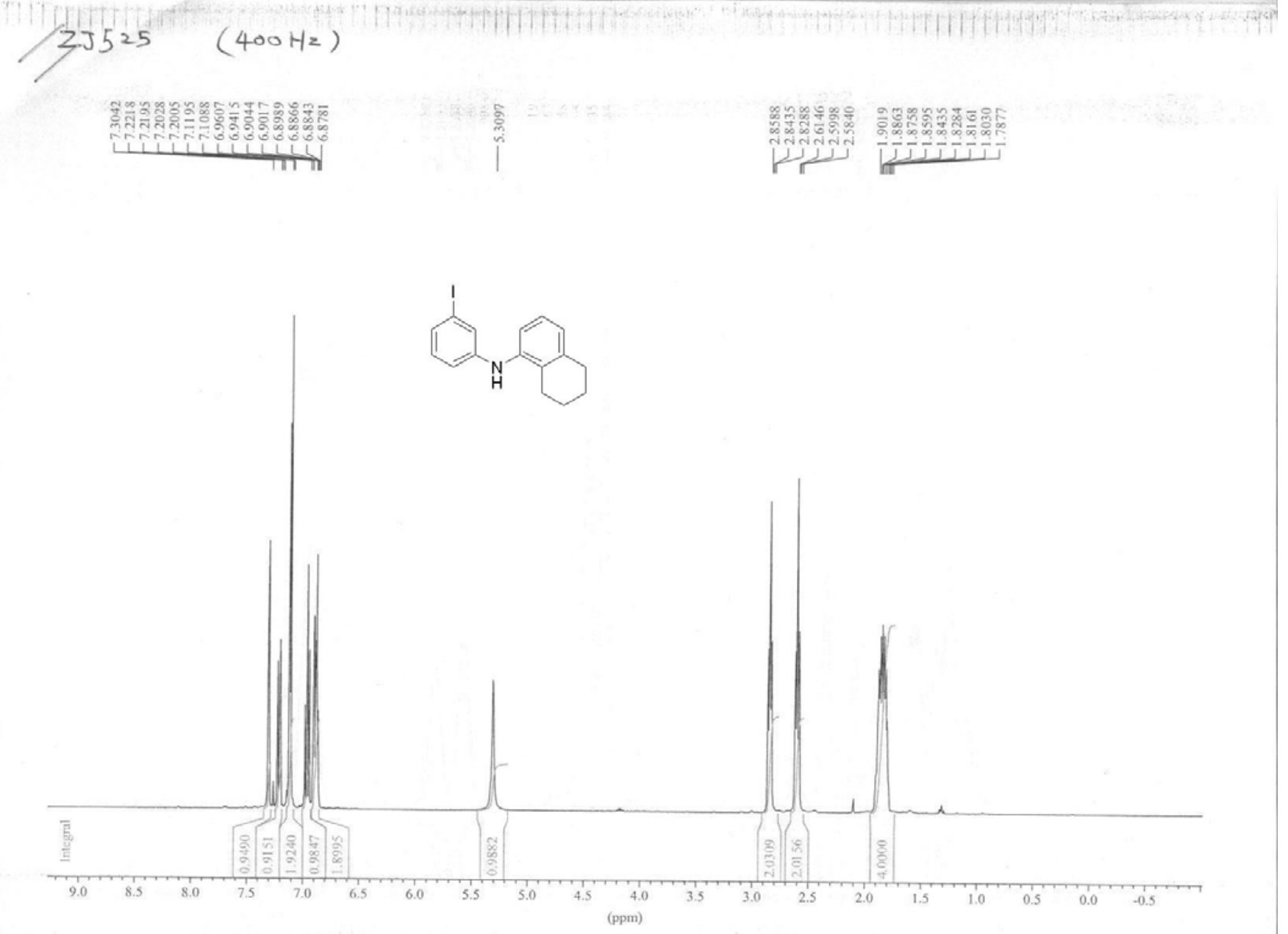

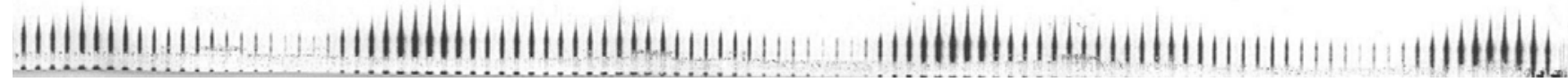




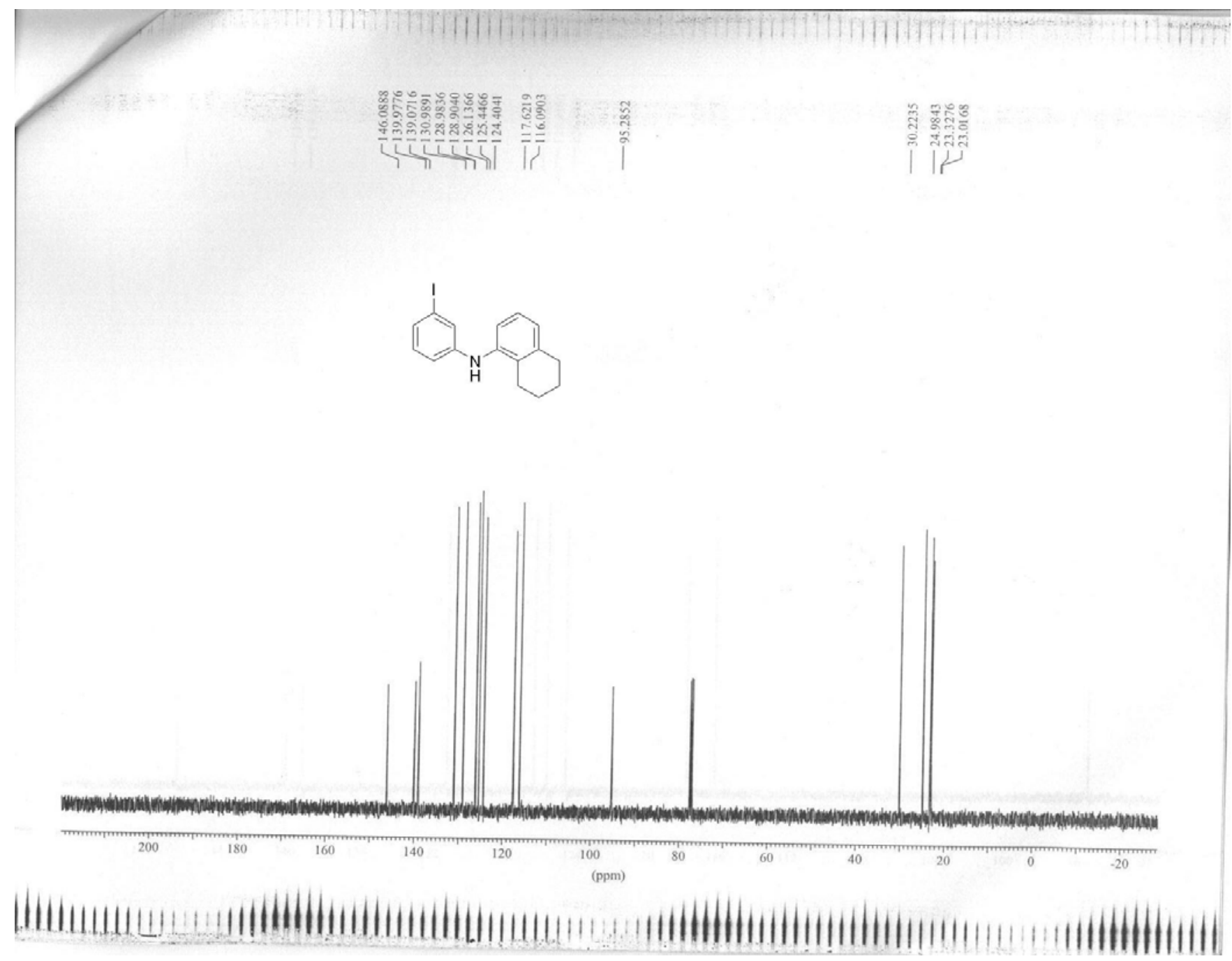




$$
25536
$$

STANDARD 1 H OBSERVE
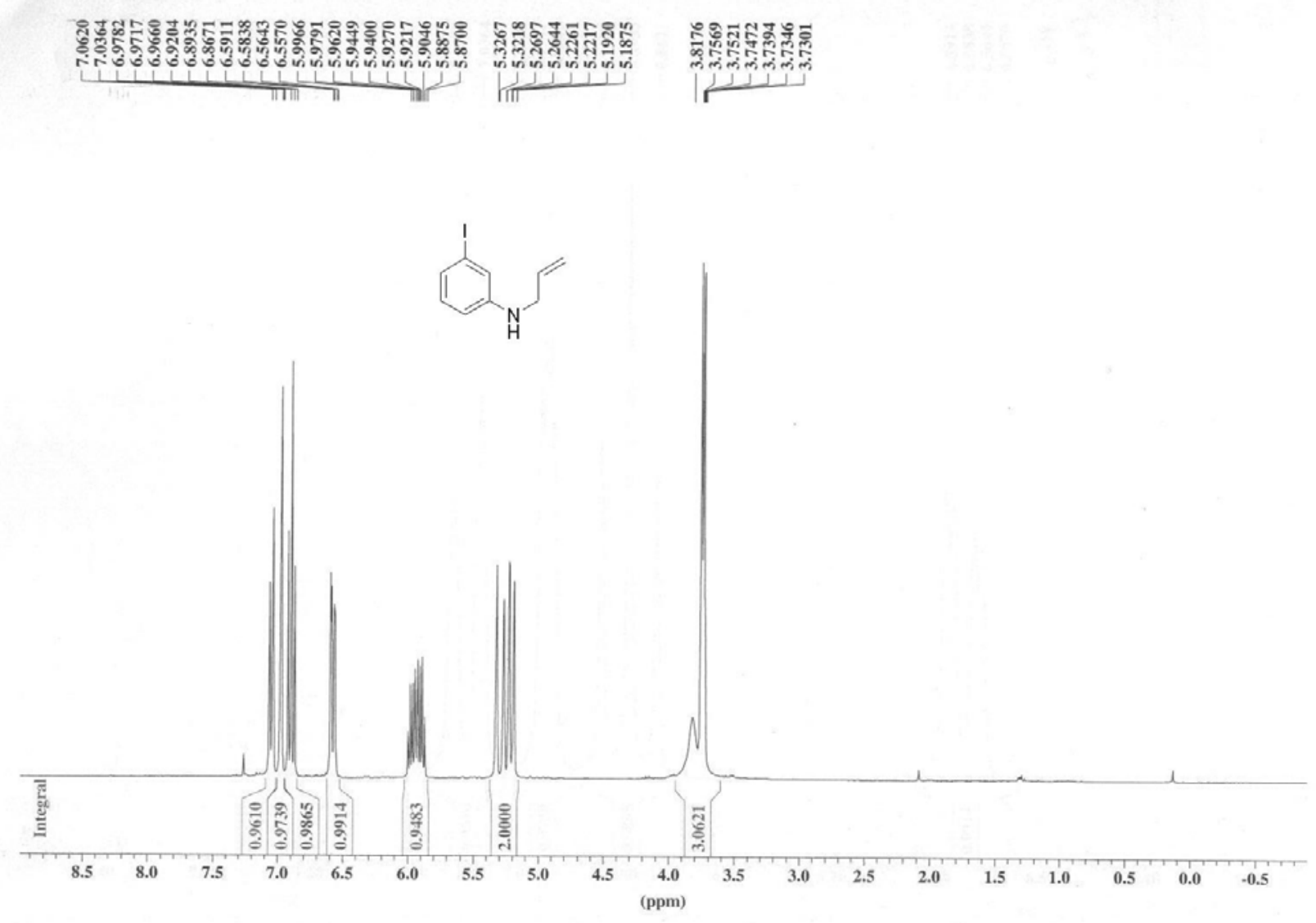


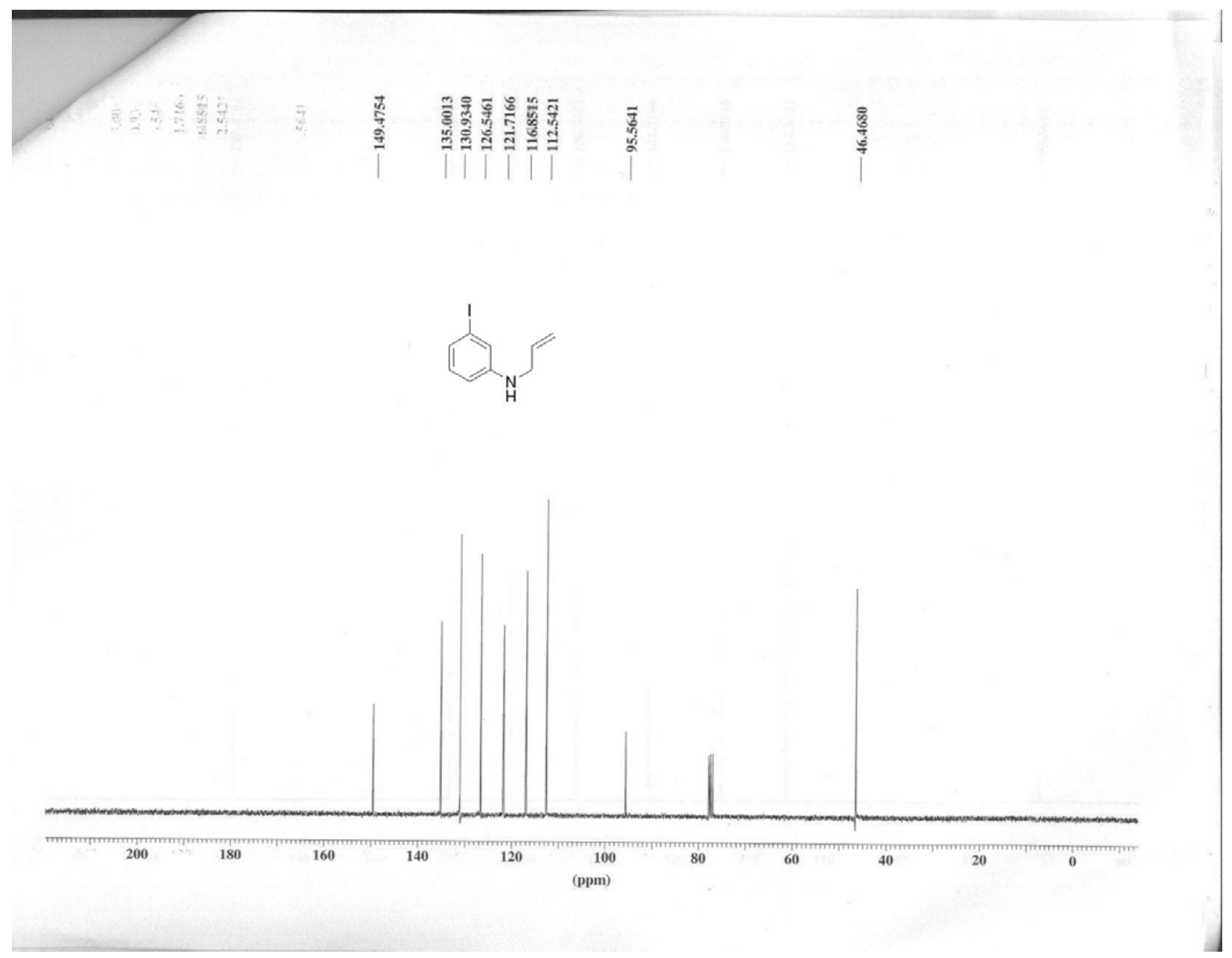


$\gamma_{2 J 555400 H:}$

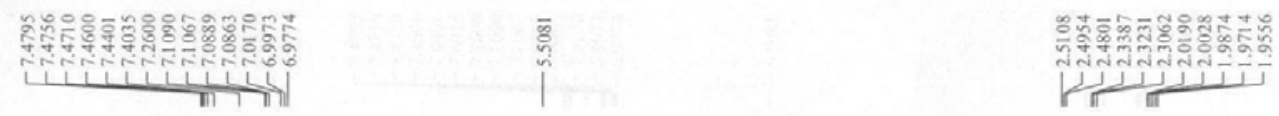

$\underbrace{(1)}_{n}$

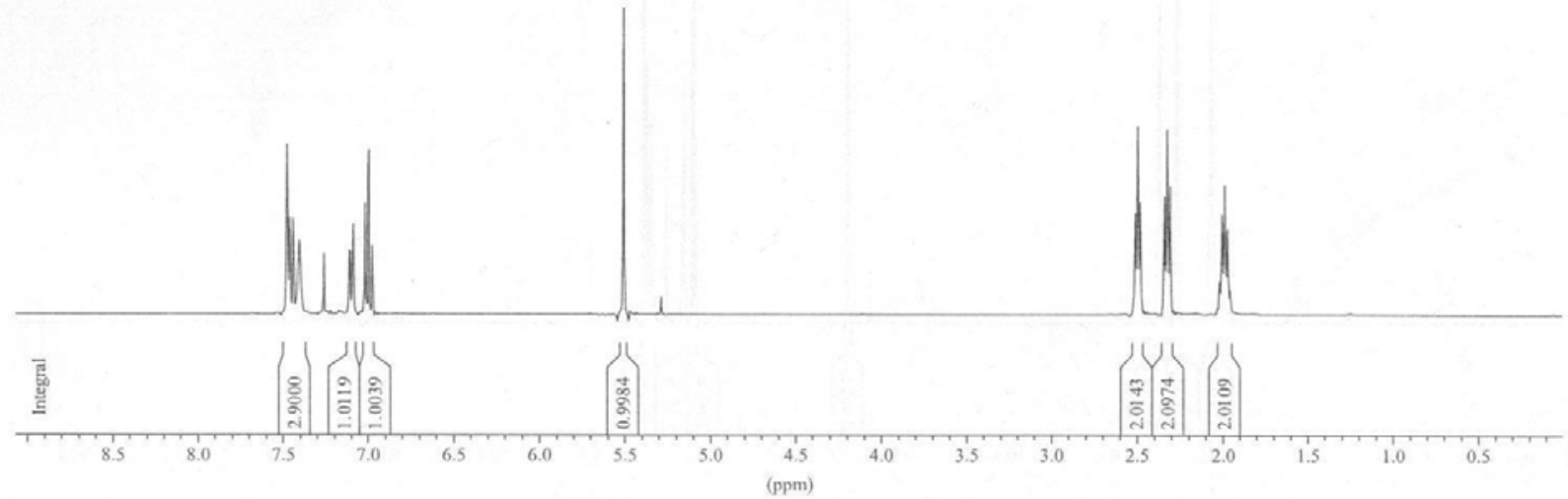




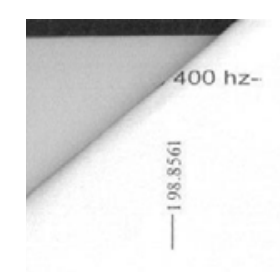

1 I

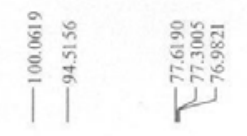

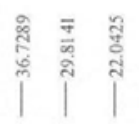
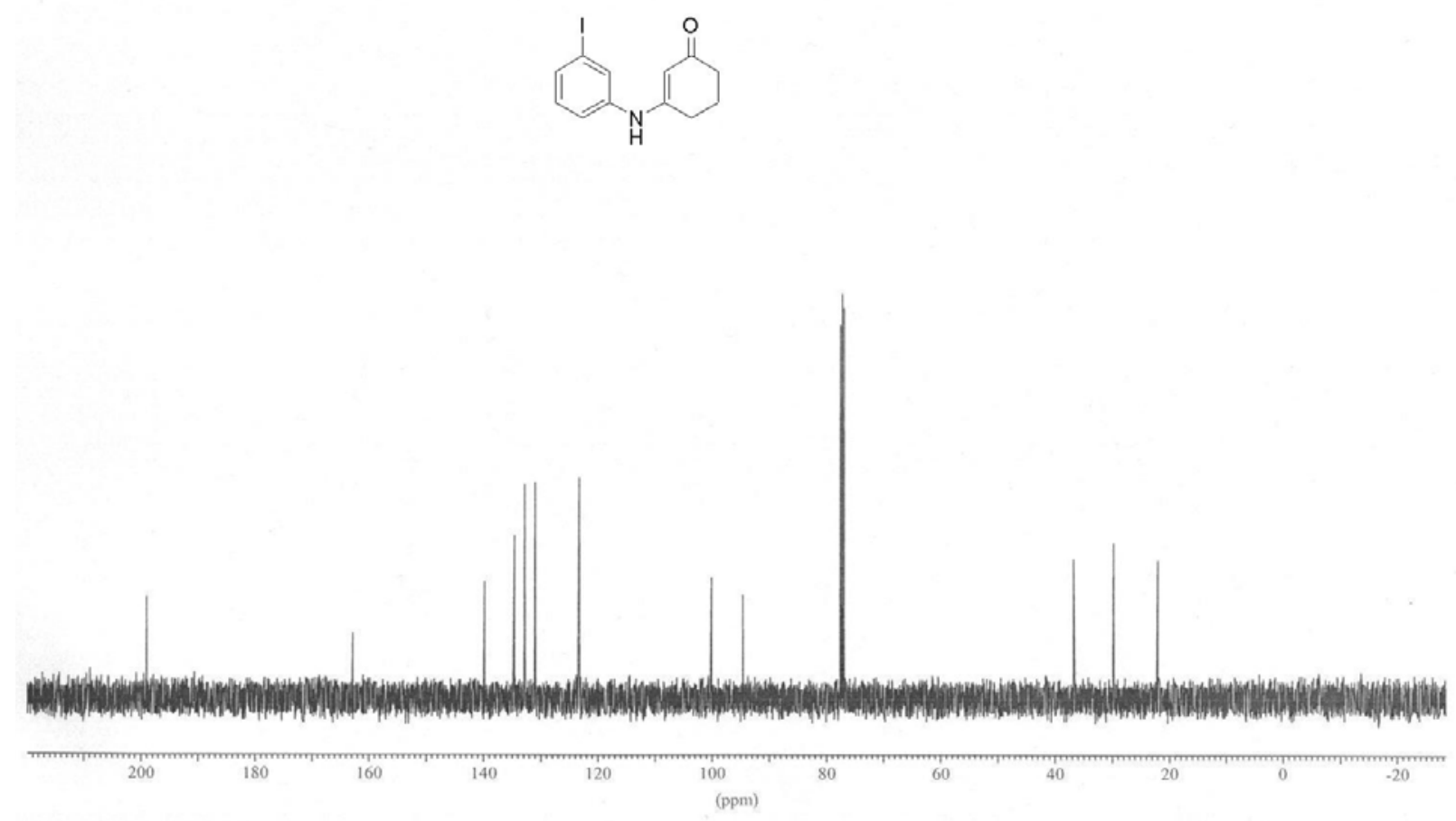
3-iodo-phenoxy benzene $400 \mathrm{~Hz}$

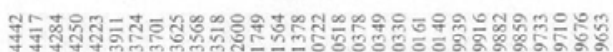

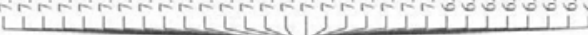

Tाาा
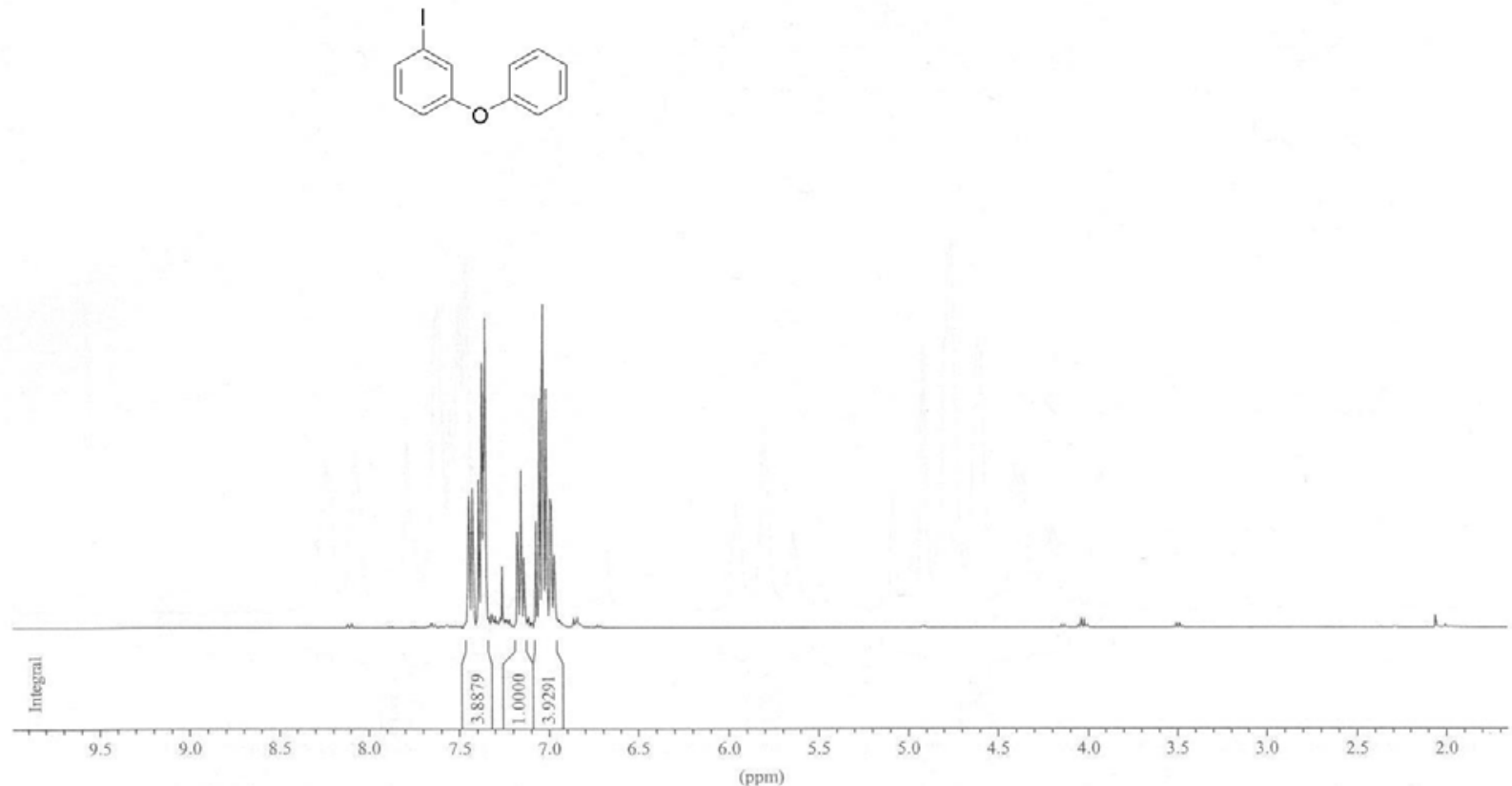


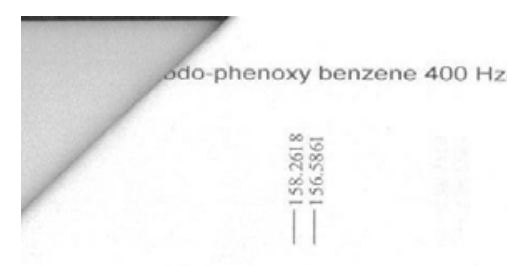

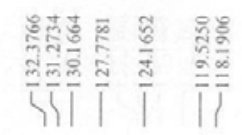

$\frac{\text { a }}{\text { | }}$
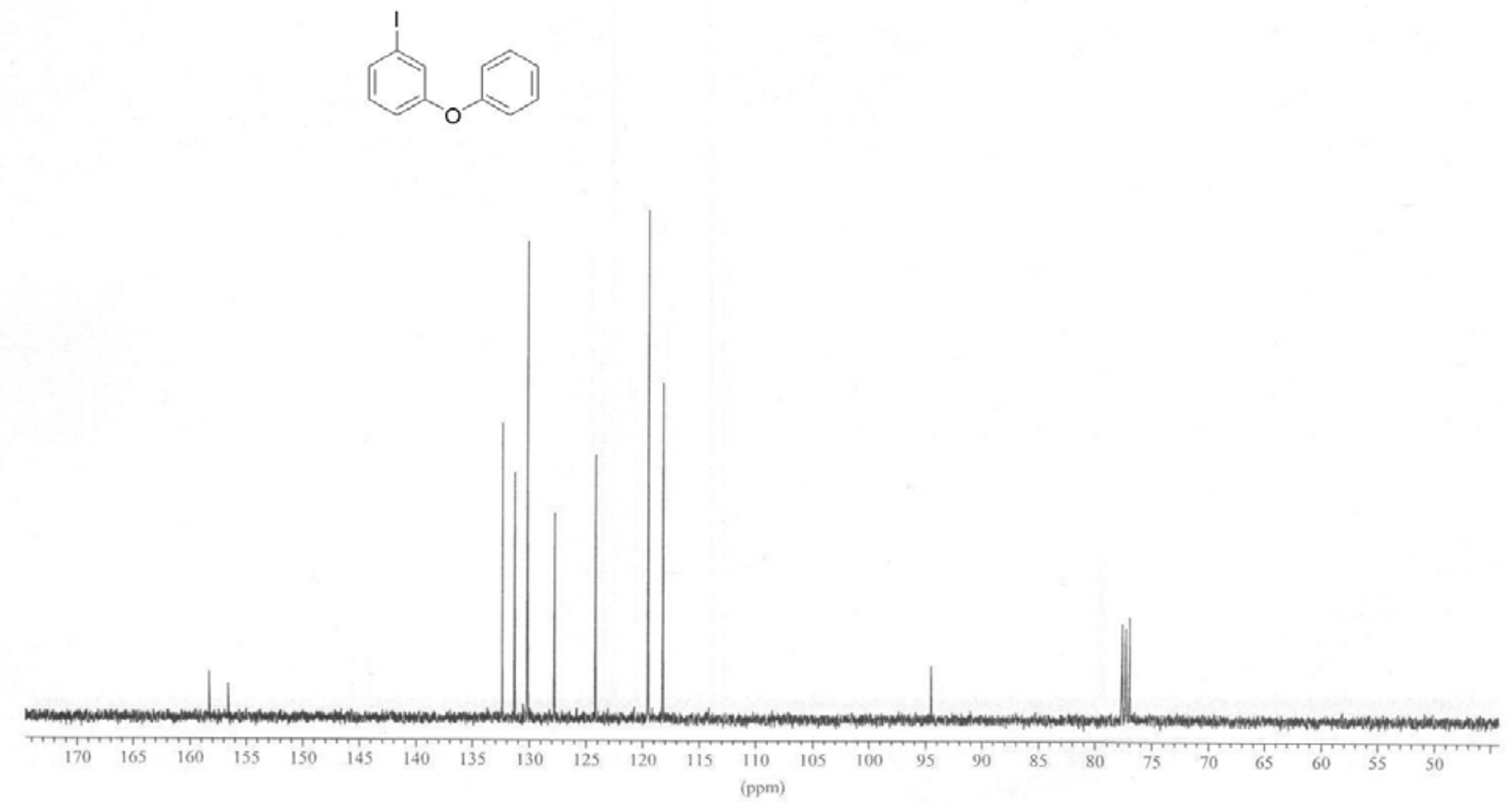
$400 \mathrm{HZ}$.
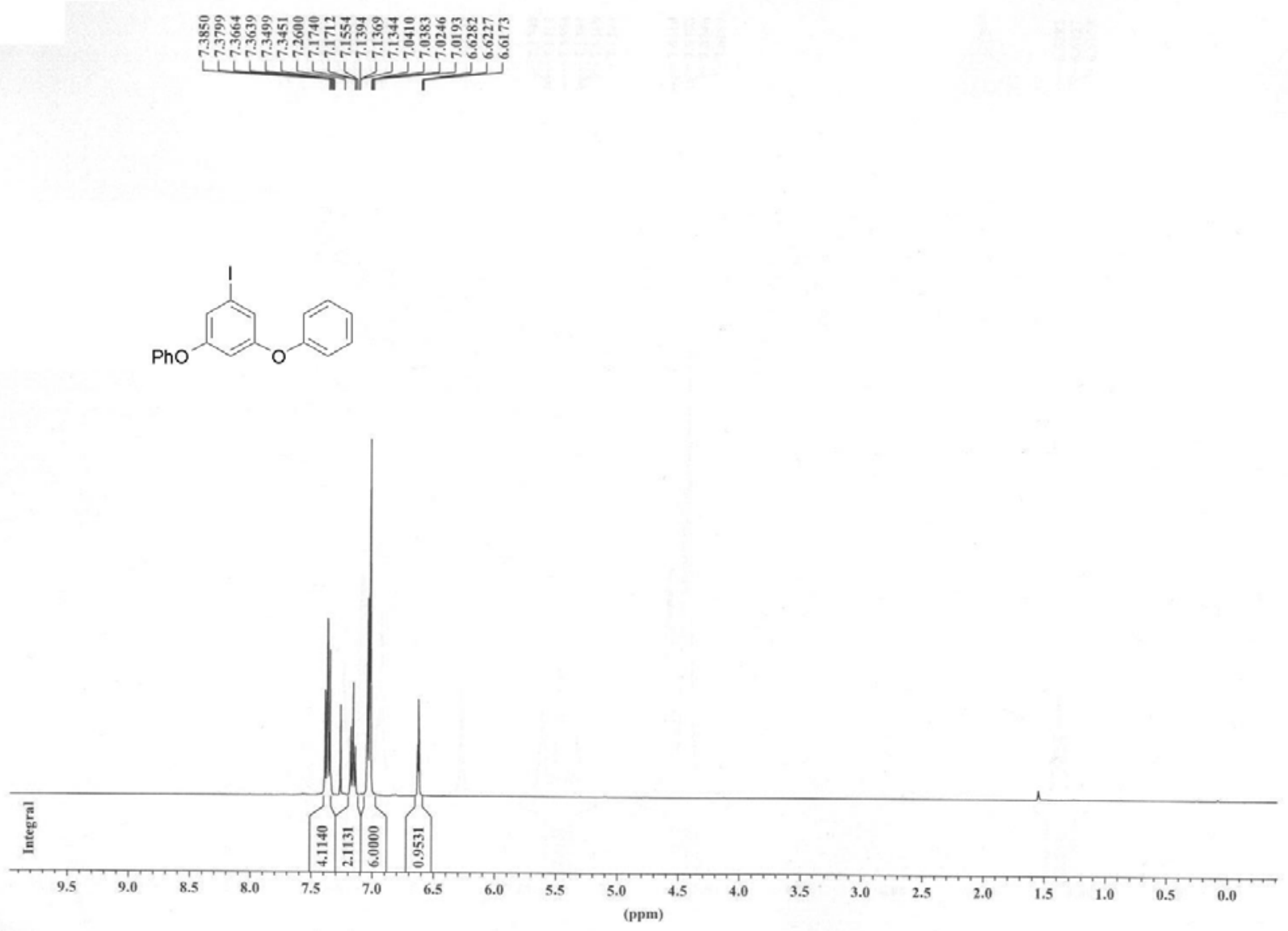


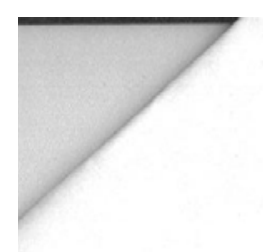

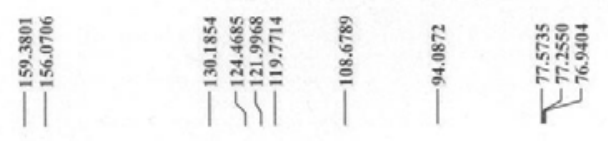
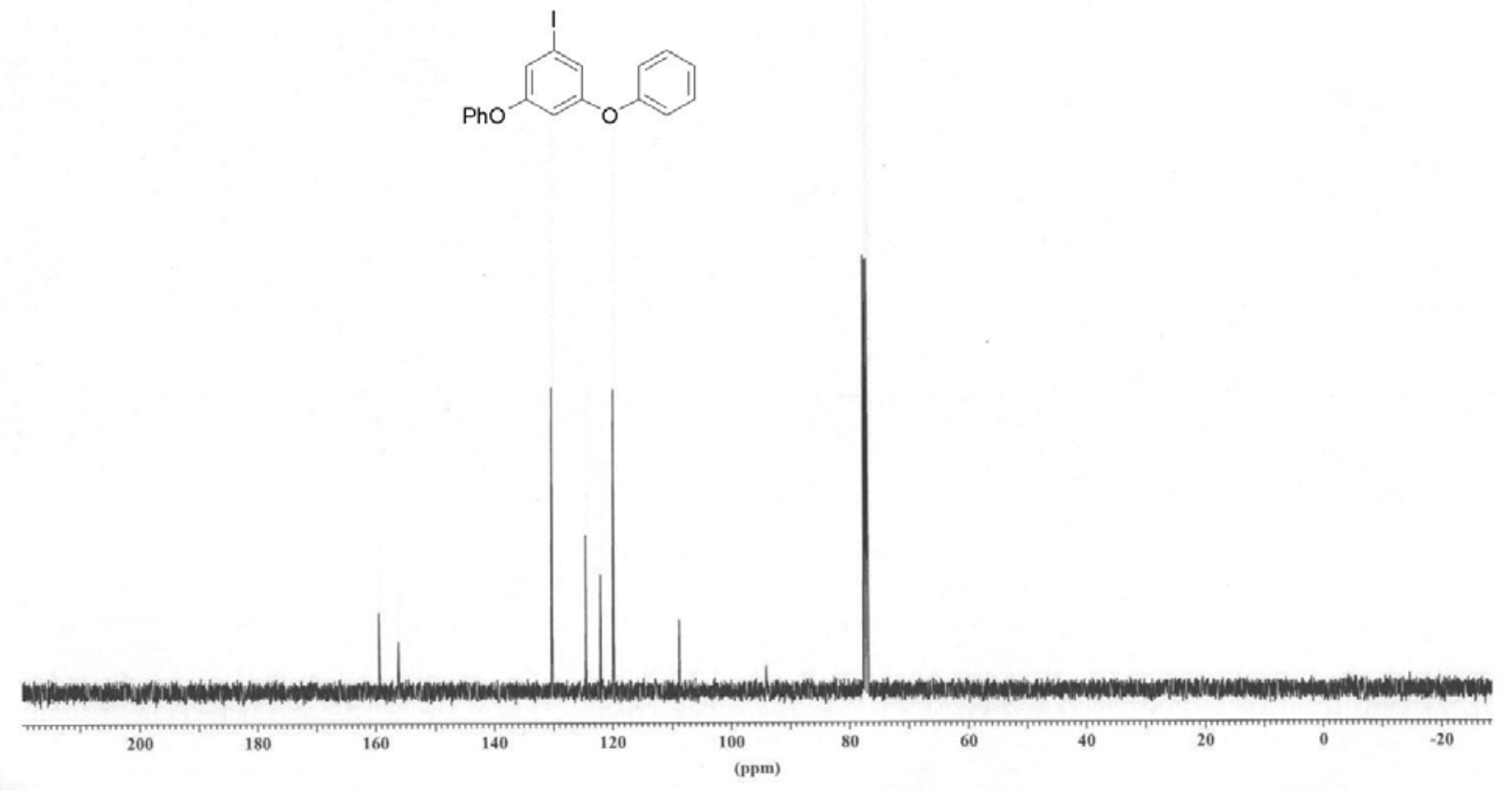
STANDARD 1H OBSERVE
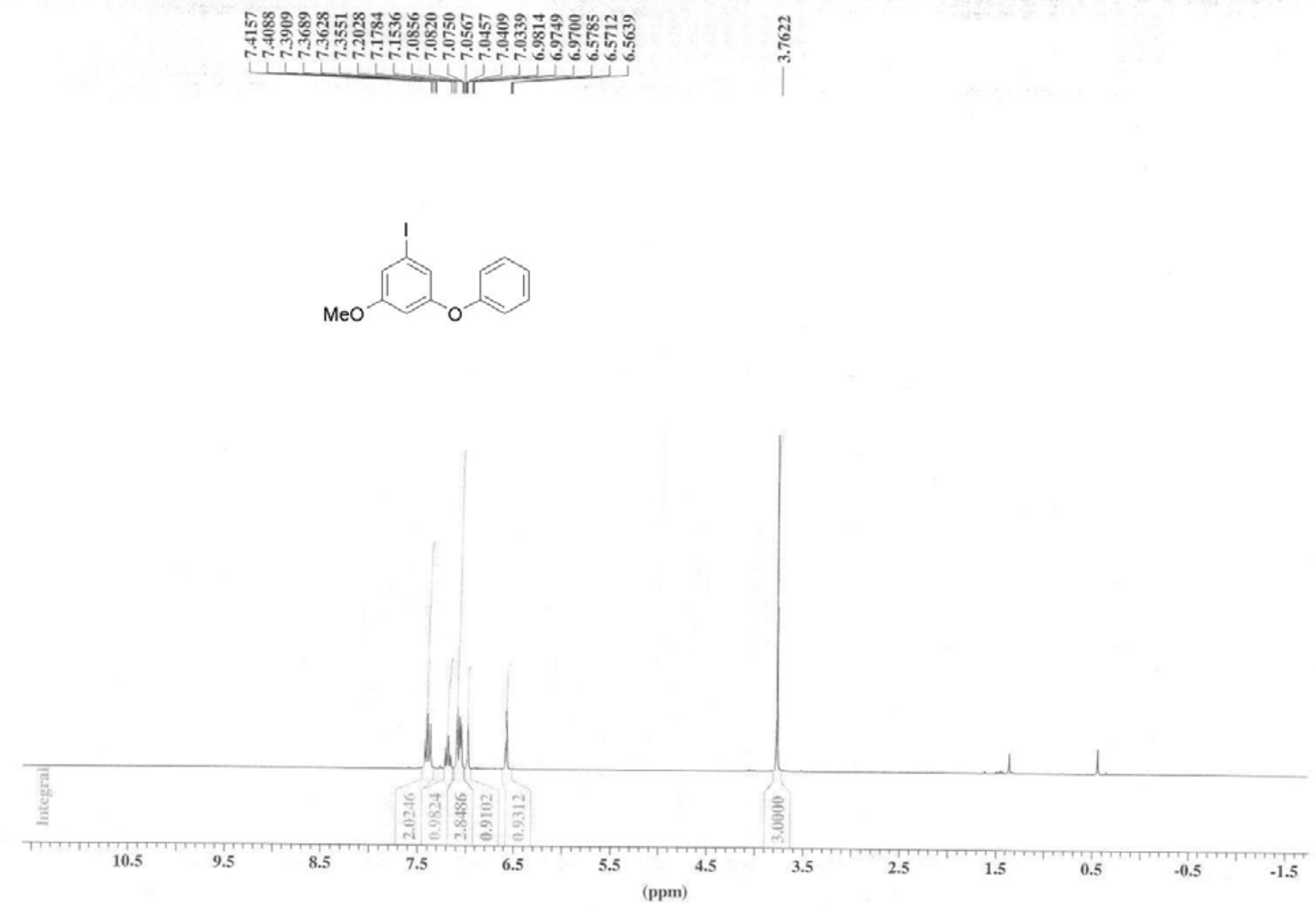


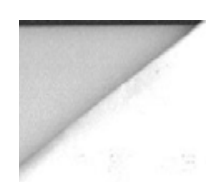

\section{ซื้กิ}

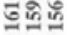

1)

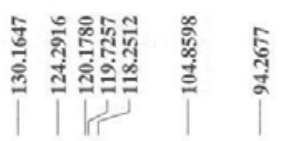

|
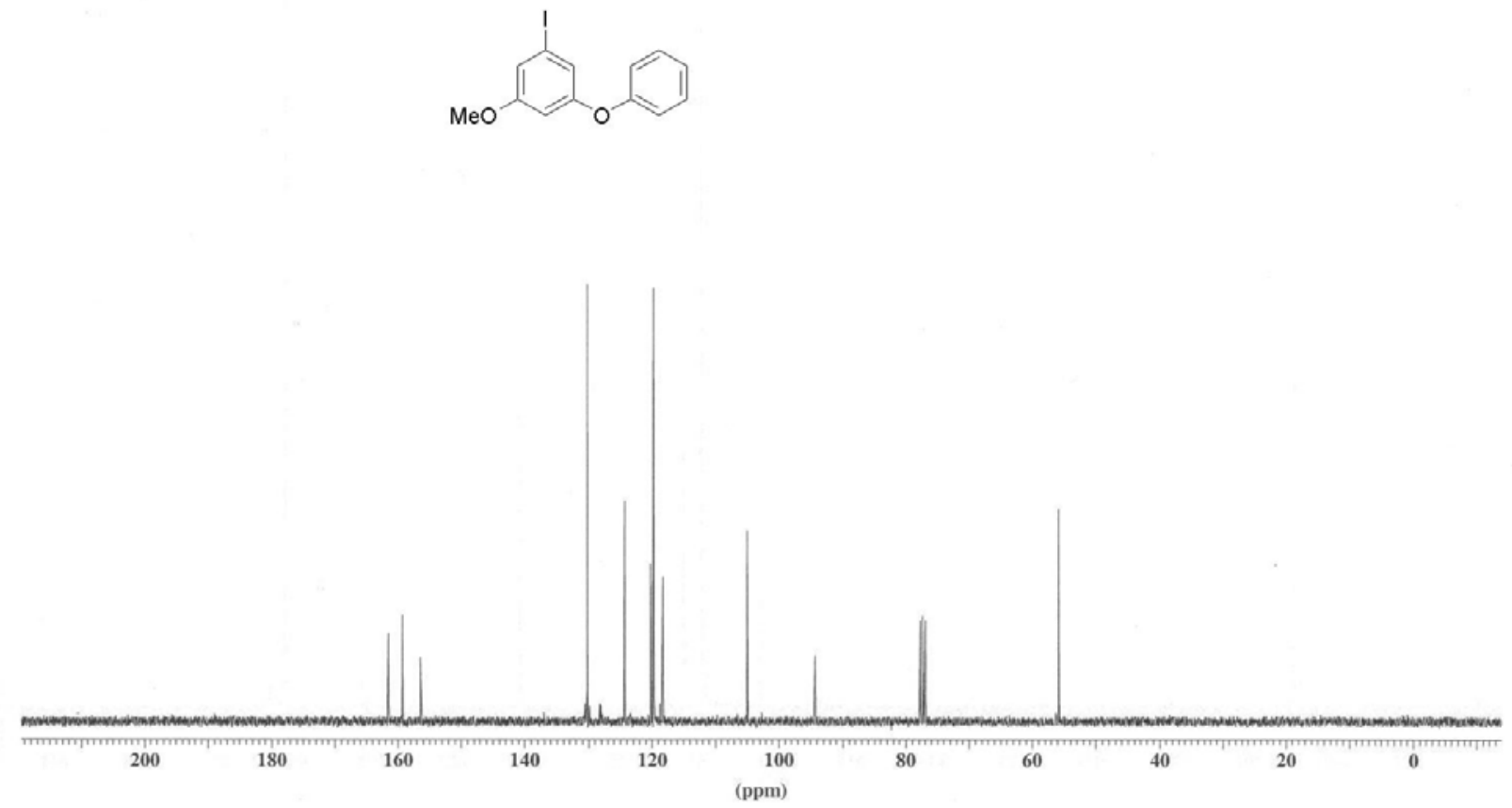


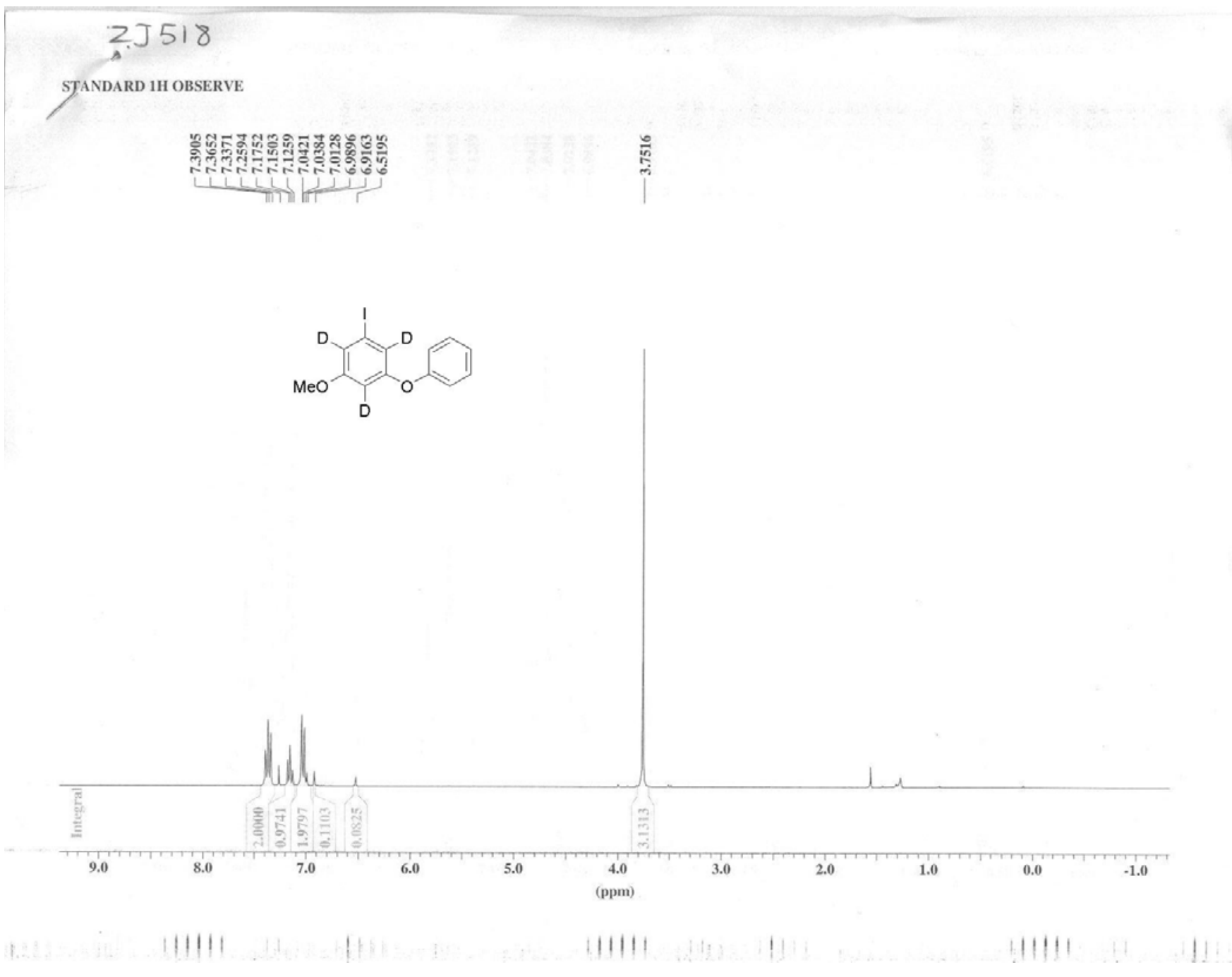




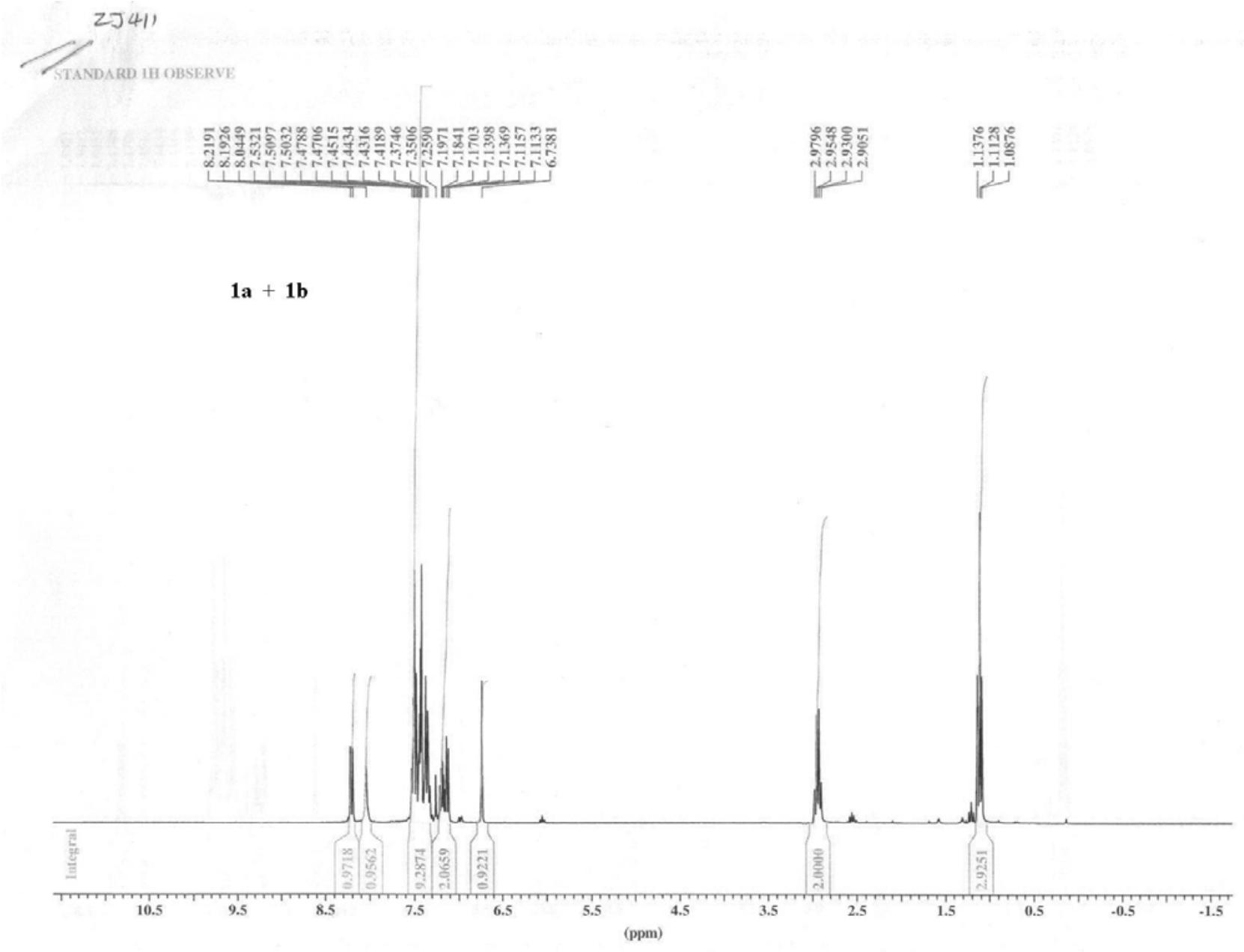



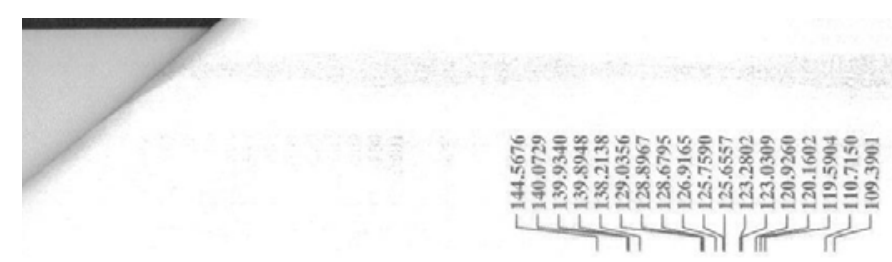

।

$1 a+1 b$

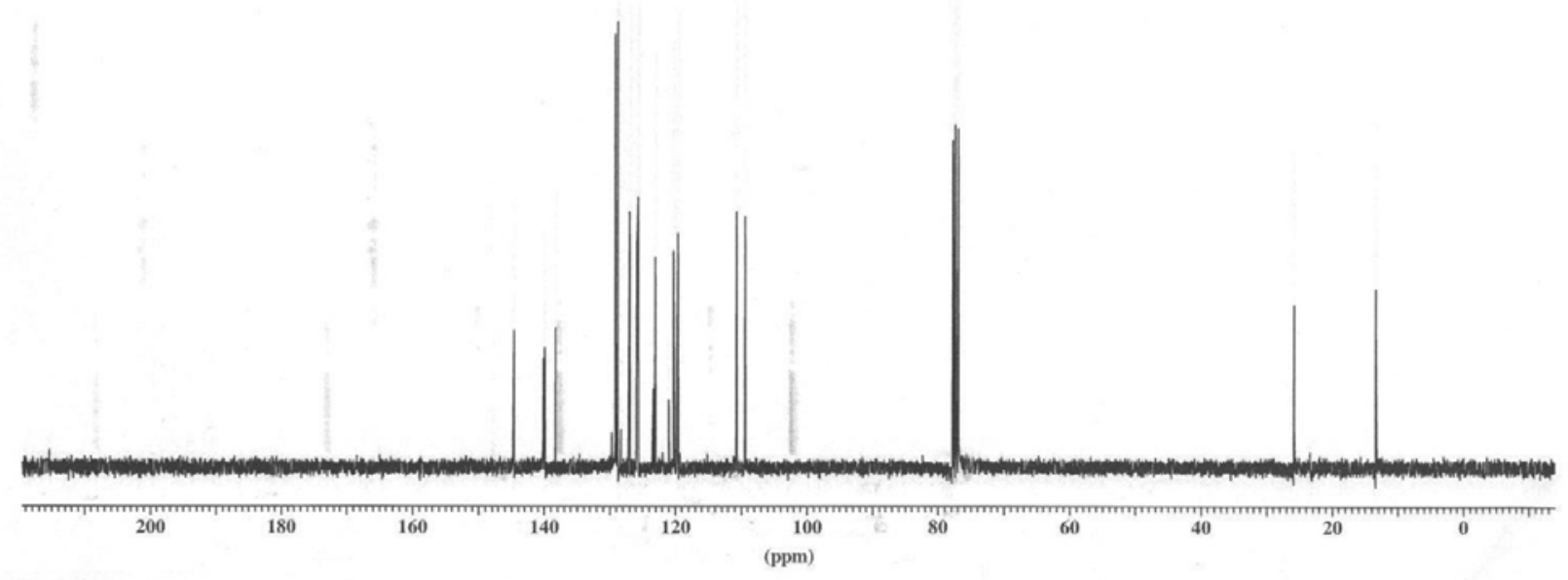


$2 J 464 A$ A STANDARD 1 H OBSERVE

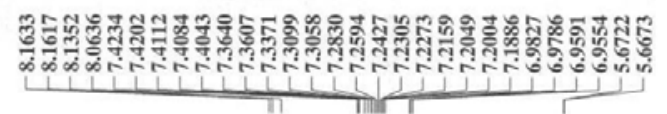

2a
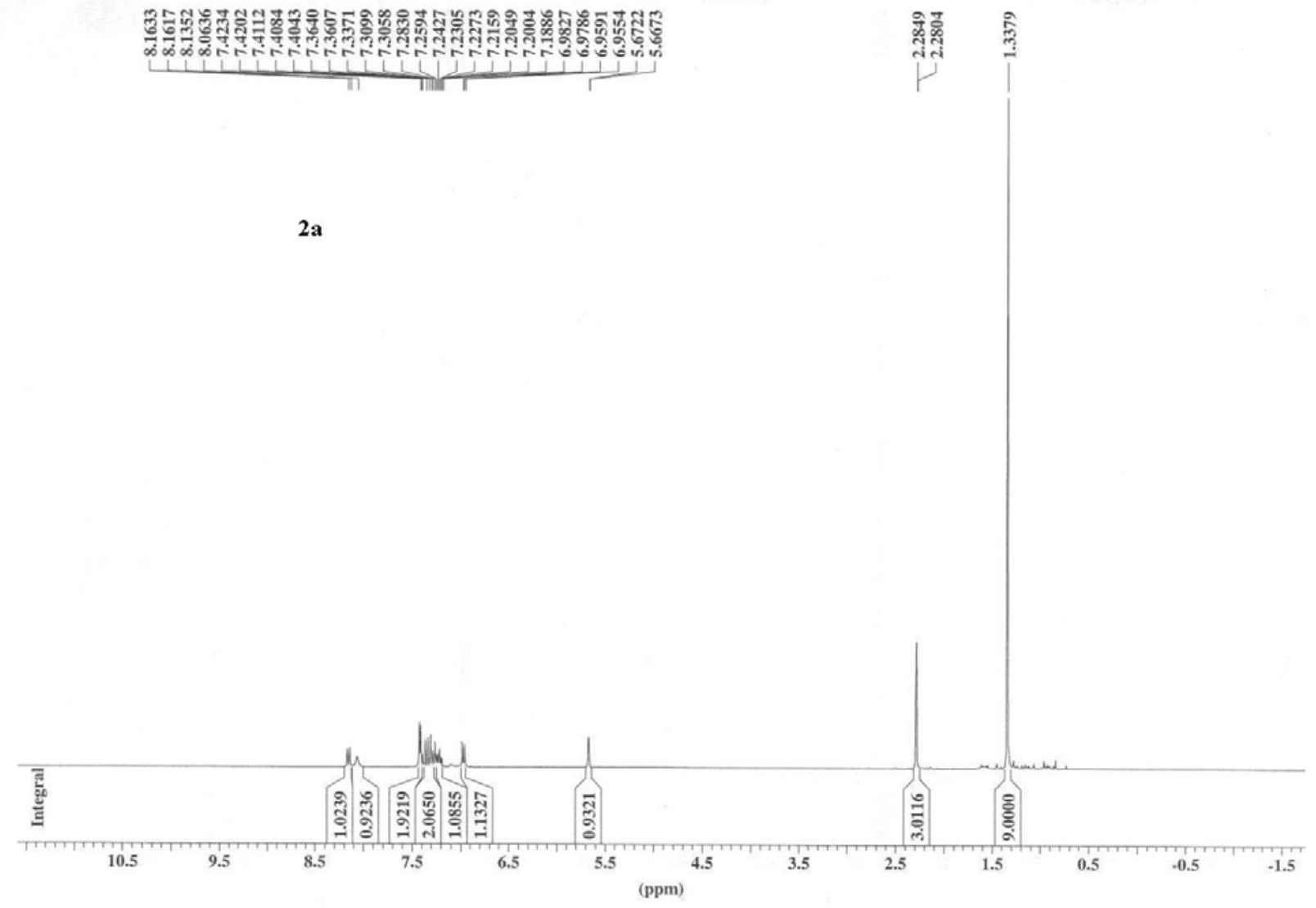

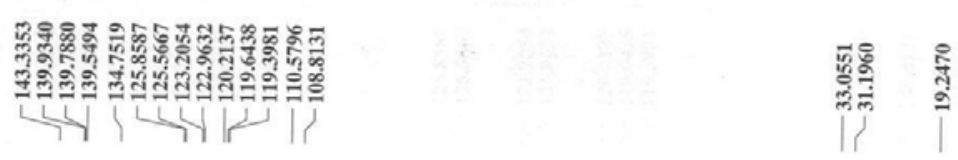

2a

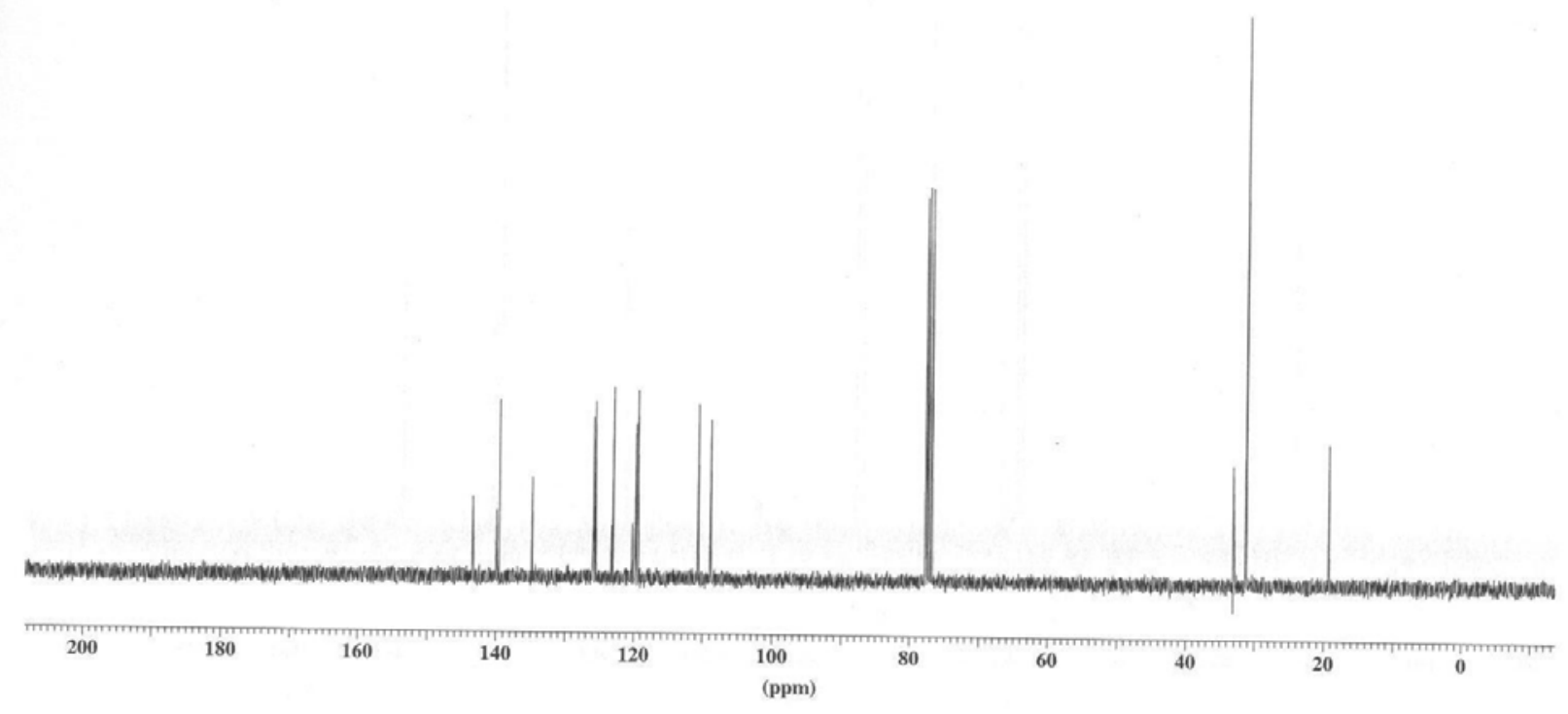


STANDARD $1 \mathrm{H}$ OBSERVE

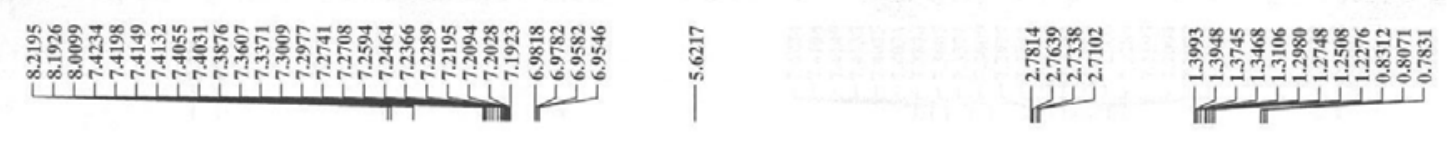

$4 a$

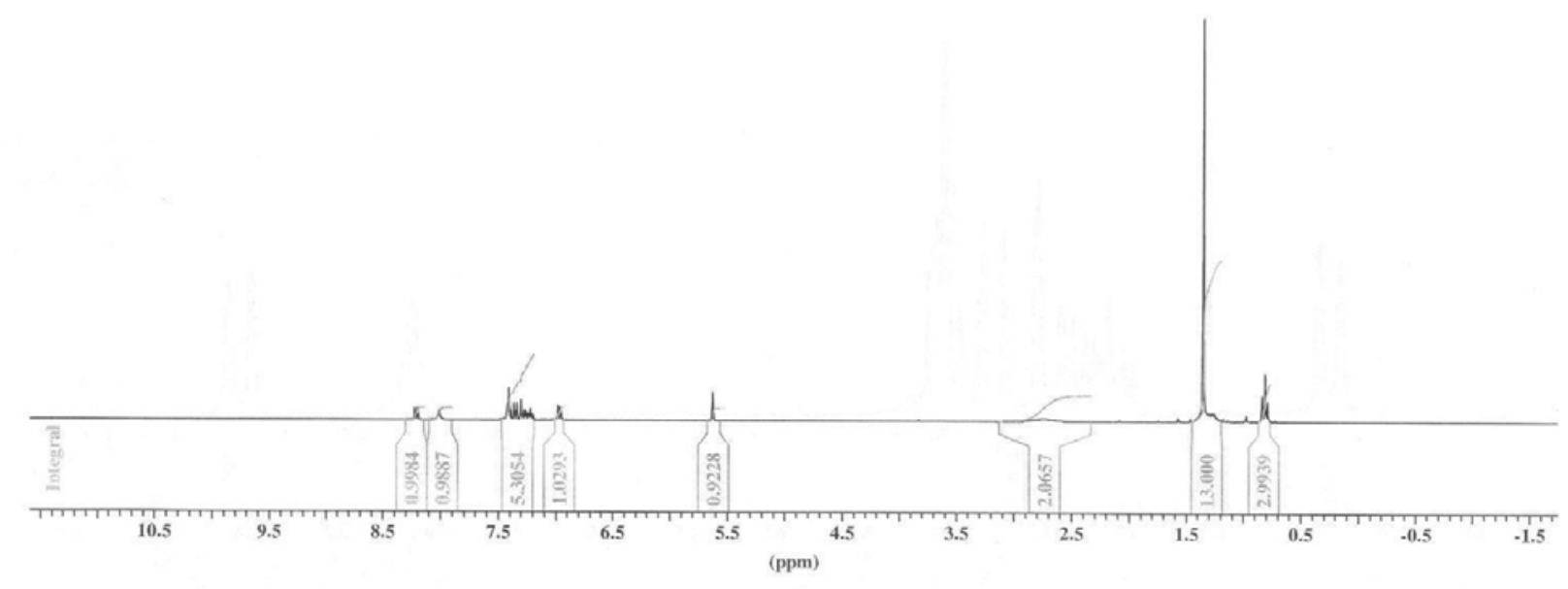

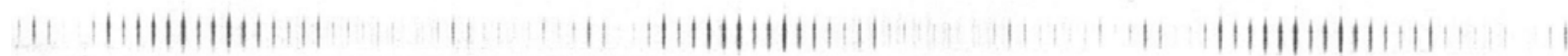



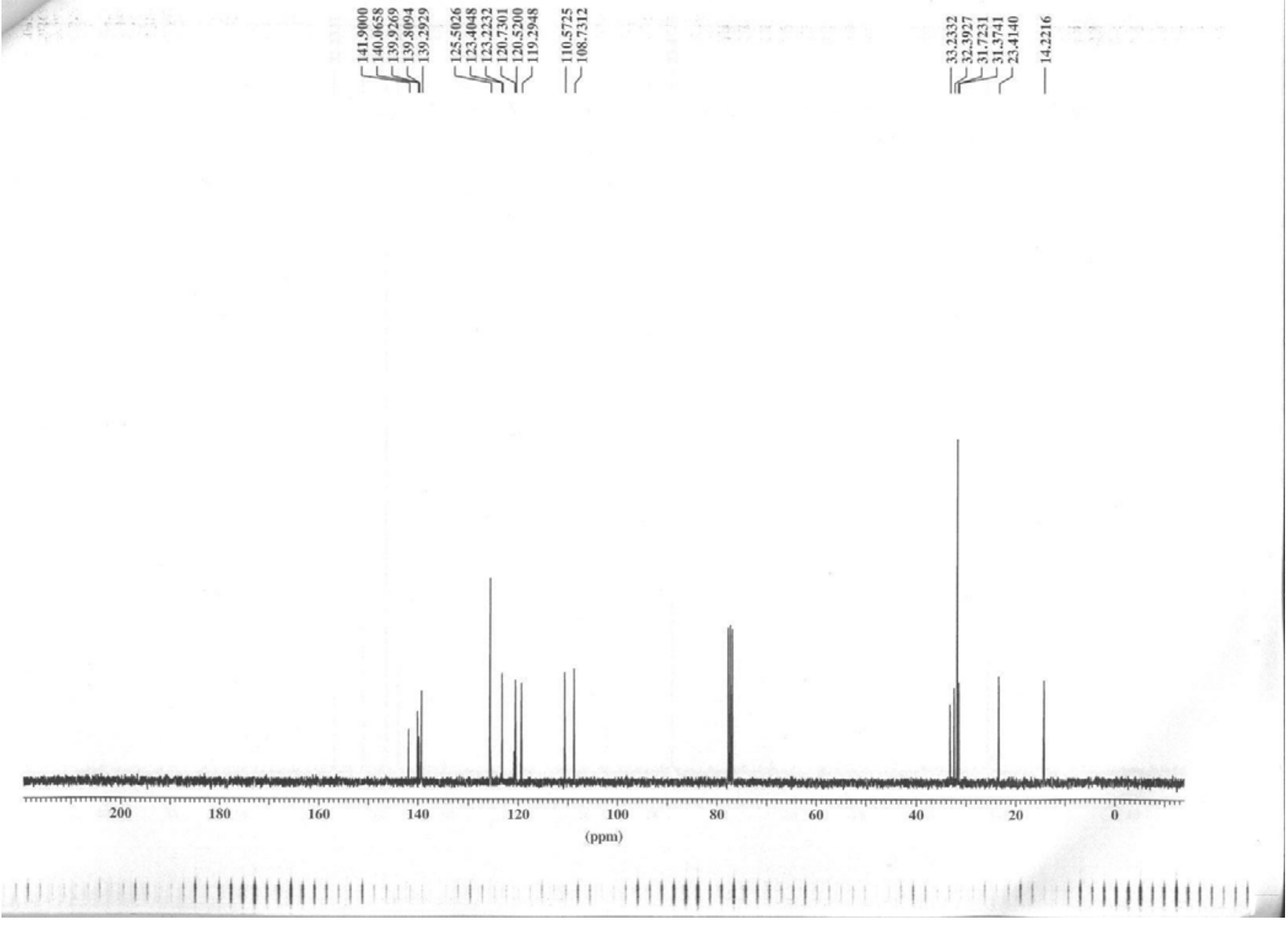
Standard IH OBSERVE zJ4 $22 \mathrm{r}$

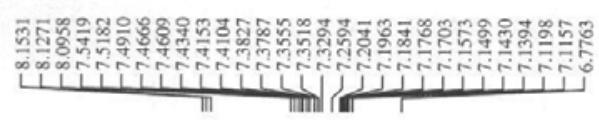

$5 \mathbf{a}+\mathbf{5 b}$

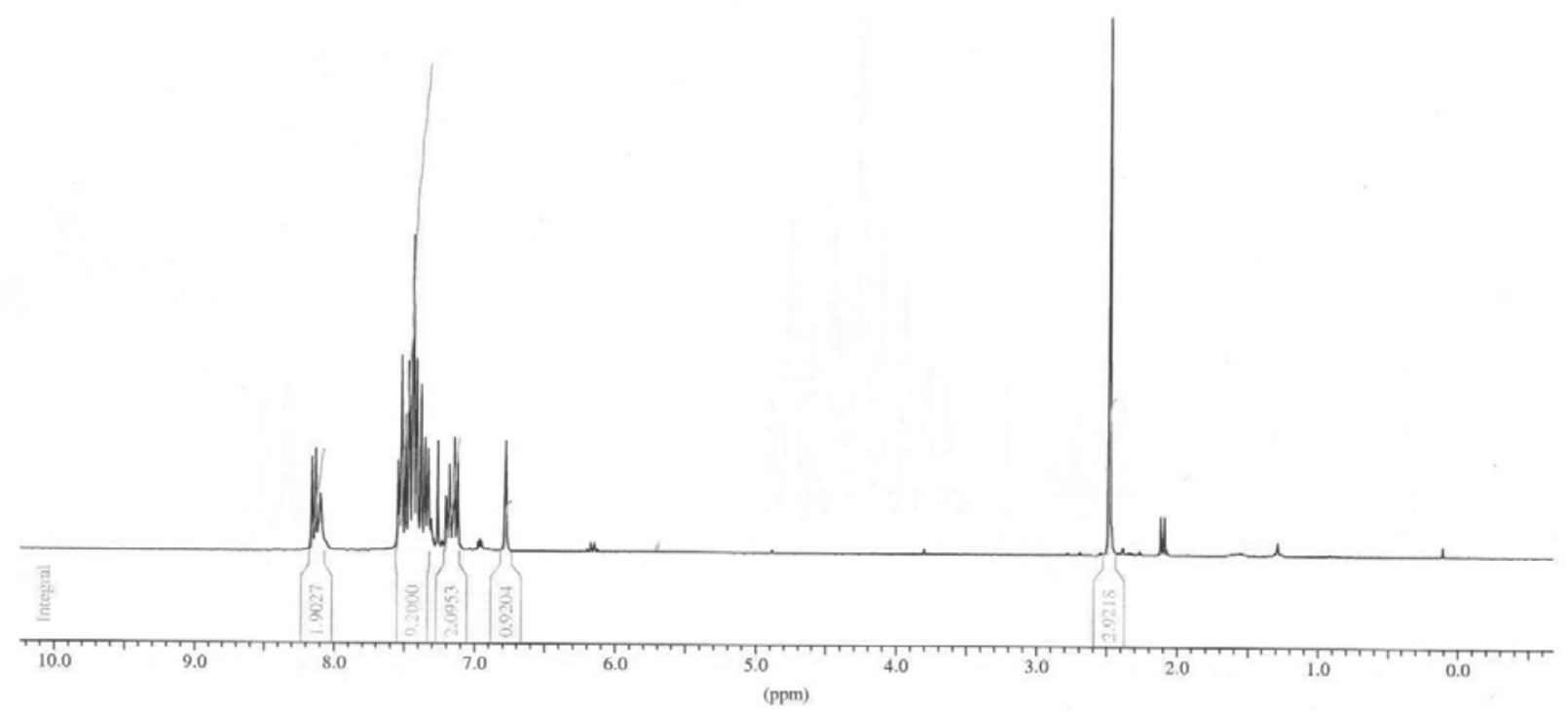




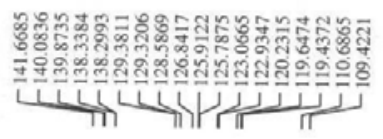

$5 \mathbf{a}+\mathbf{5 b}$

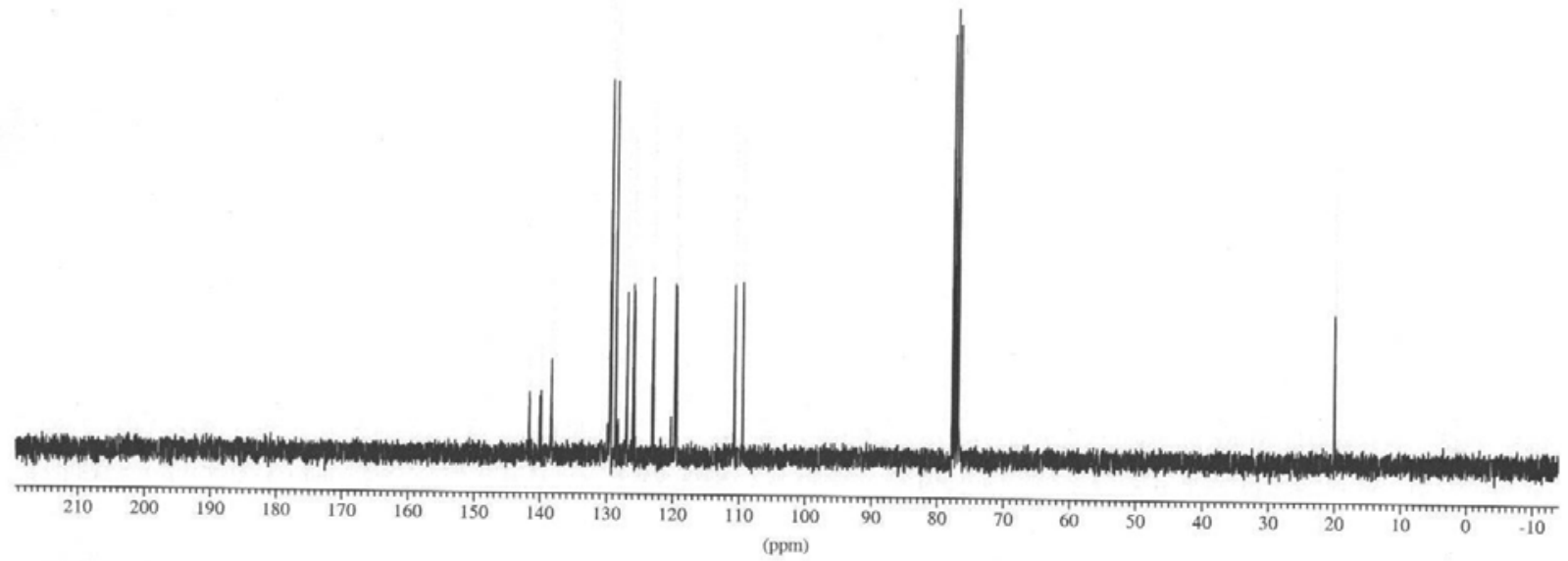


$2 J 5 \circ 9$

STANDARD $1 H$ OBSERVE

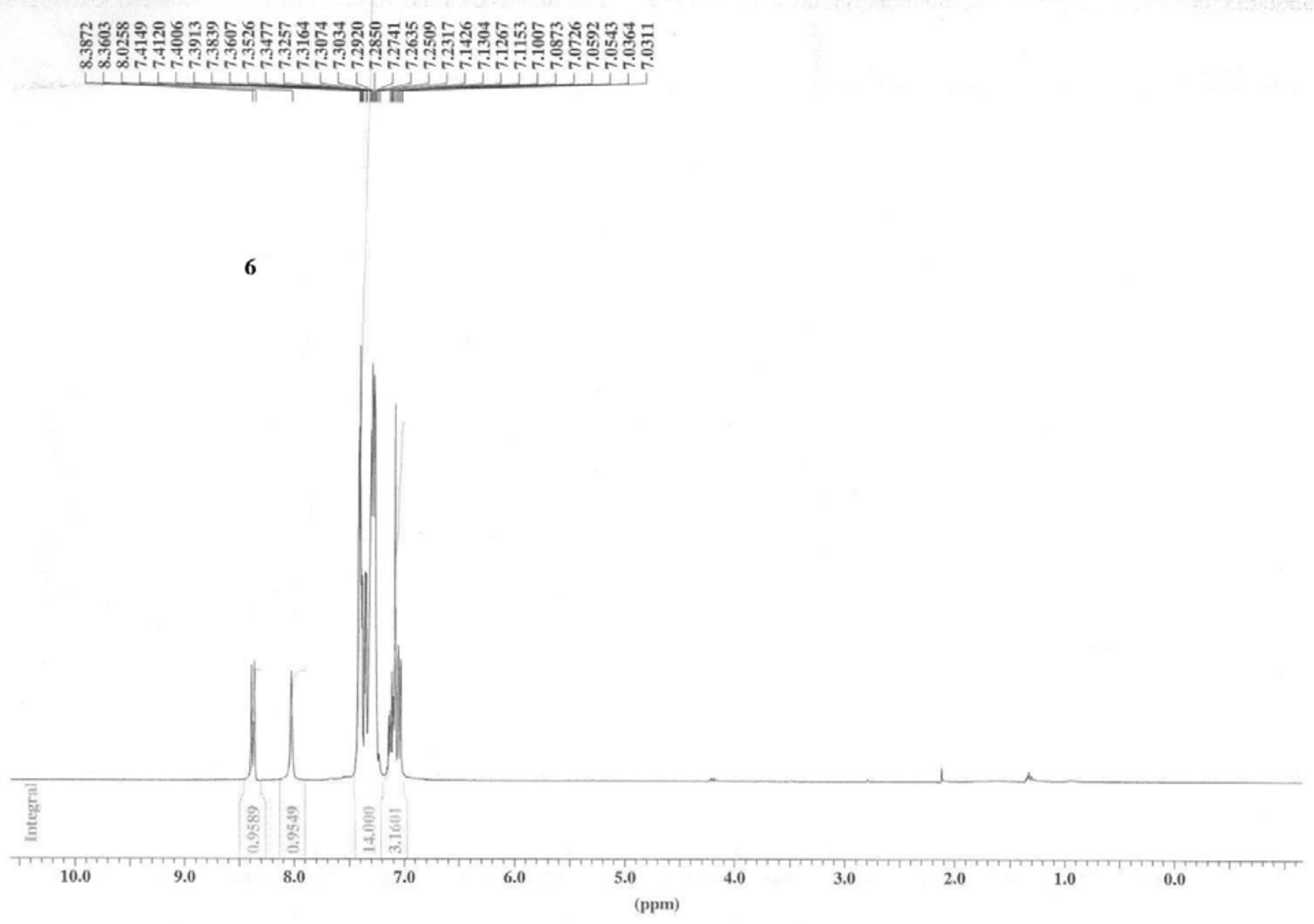



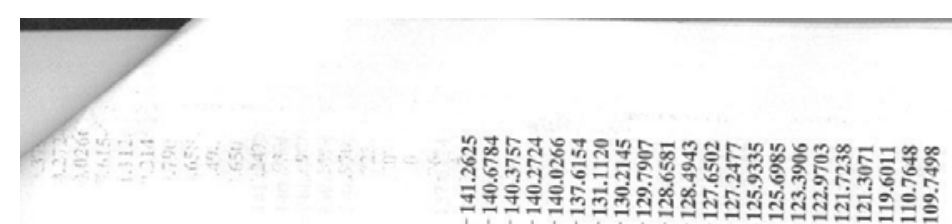

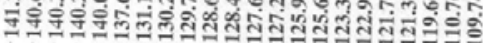
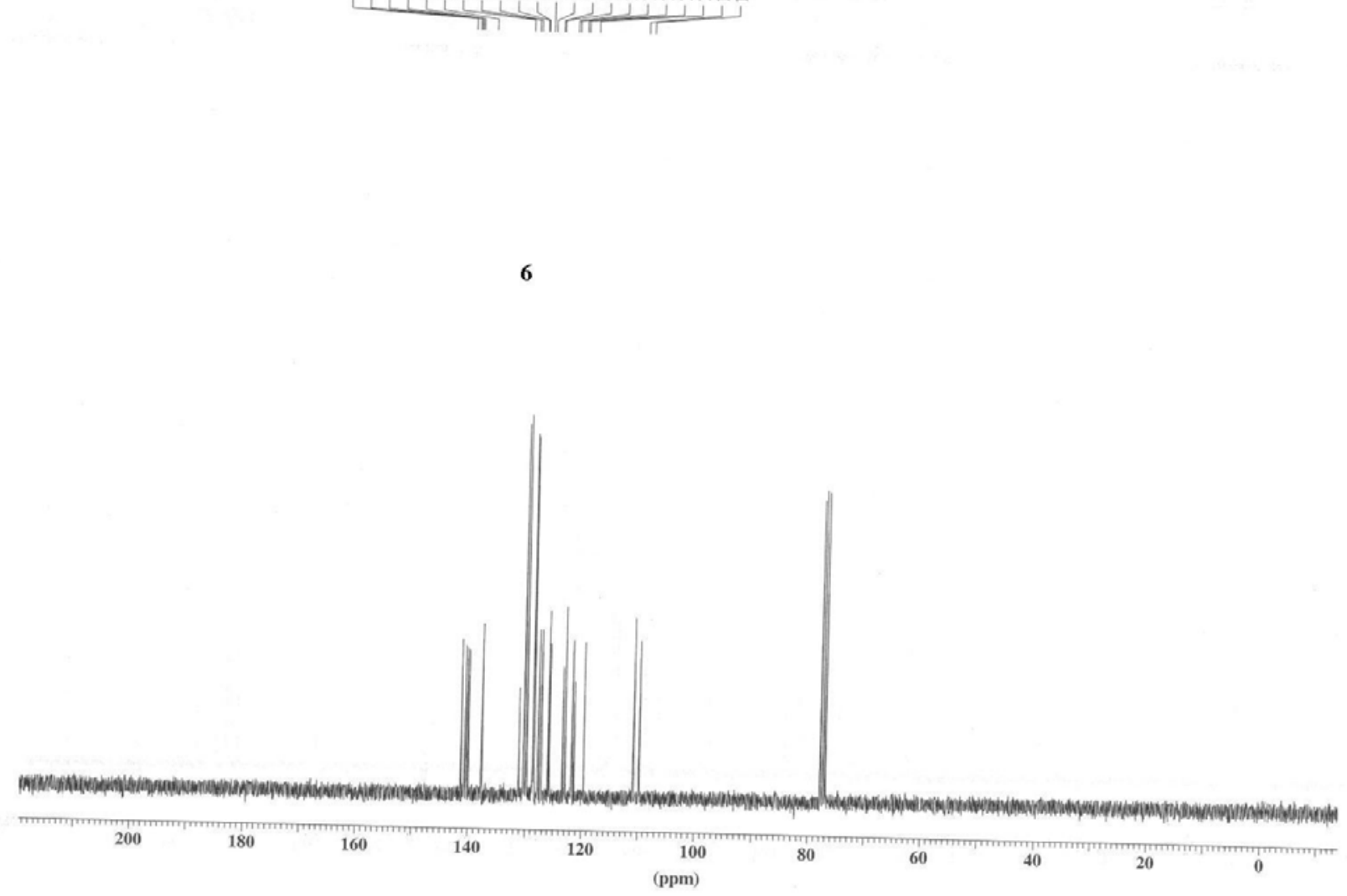
$2 J 471 B$

STANDARD 1 H OBSERVE

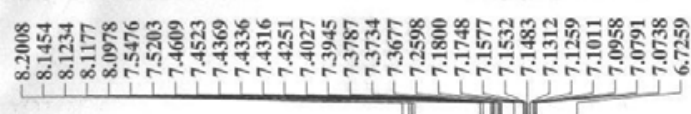

$7 \mathbf{a}$
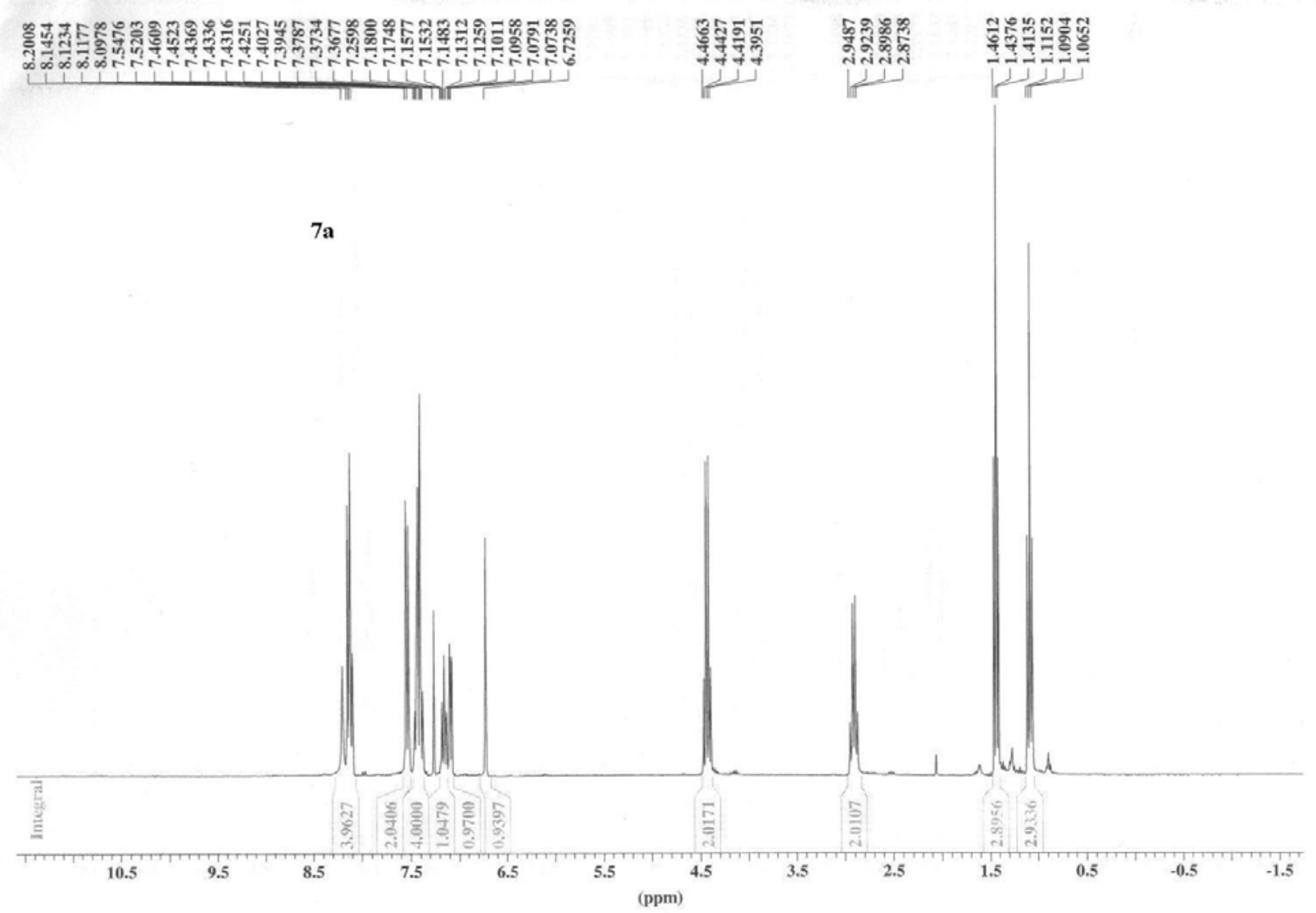


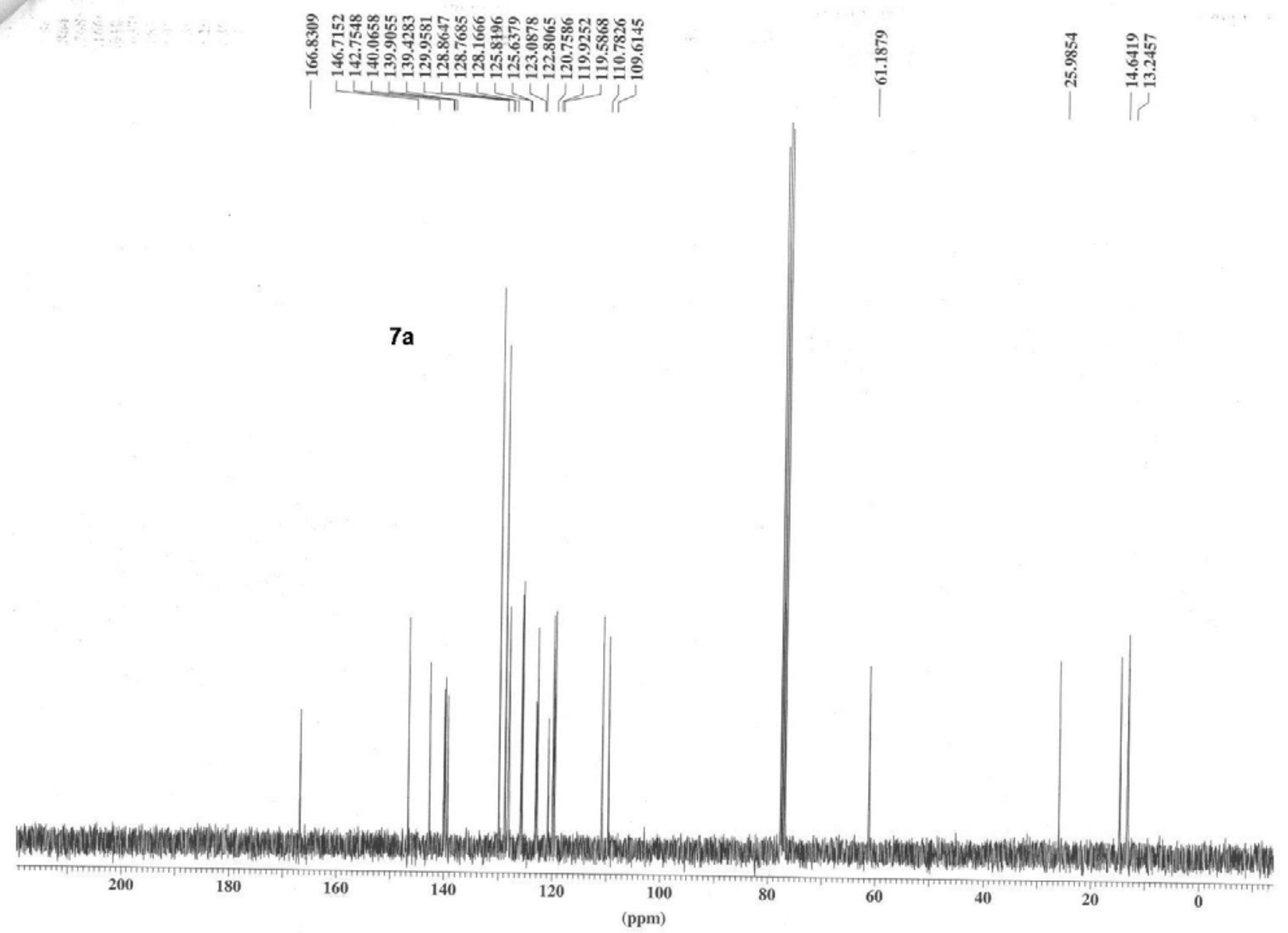


$2 J 489 B$

STANDARD 1 H OBSERVE

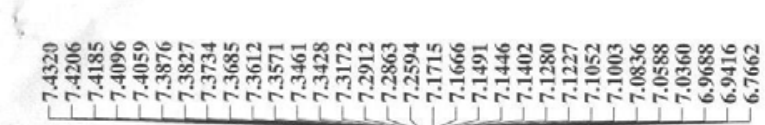

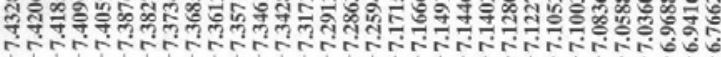

IIII) |ाIIII।

-

$8 a$

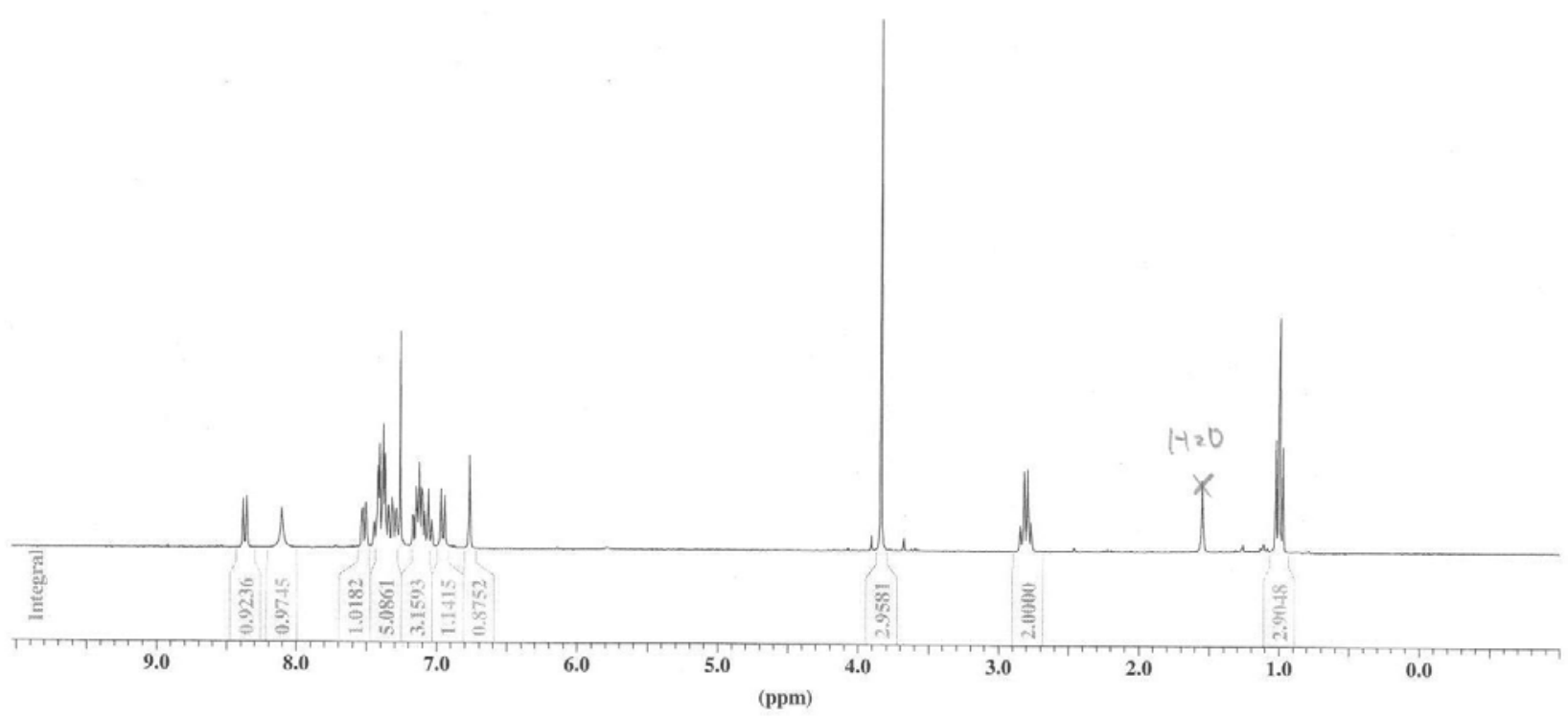




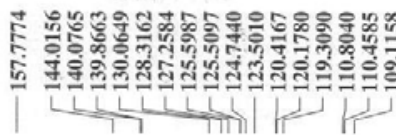

की

$8 a$

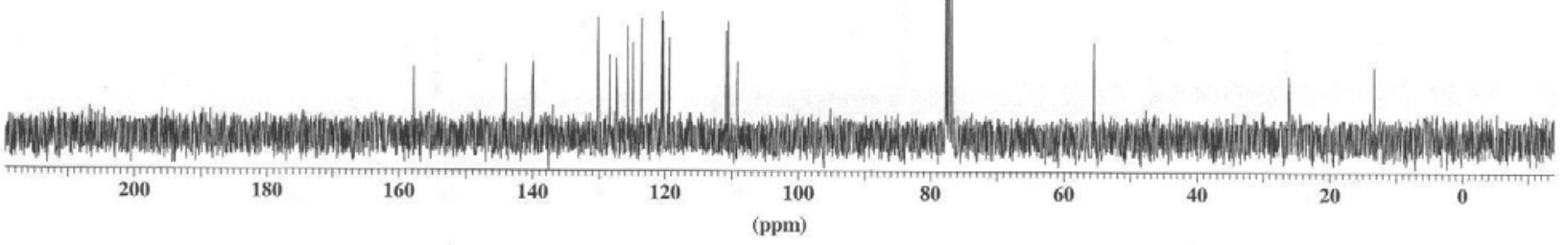


Z z 510

STANDARD $1 \mathrm{H}$ OBSERVE

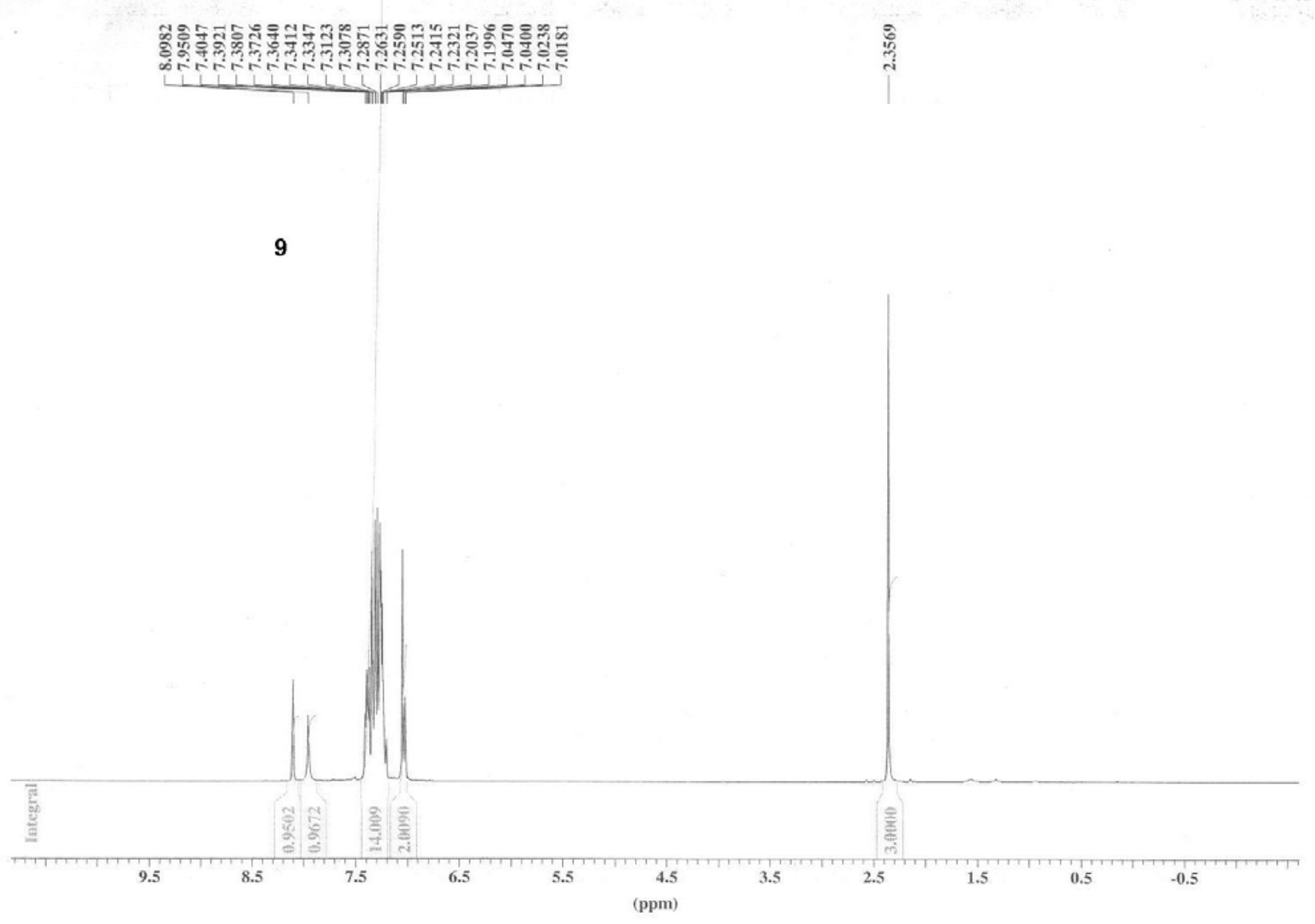




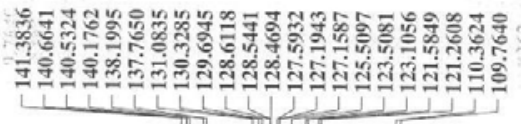

9

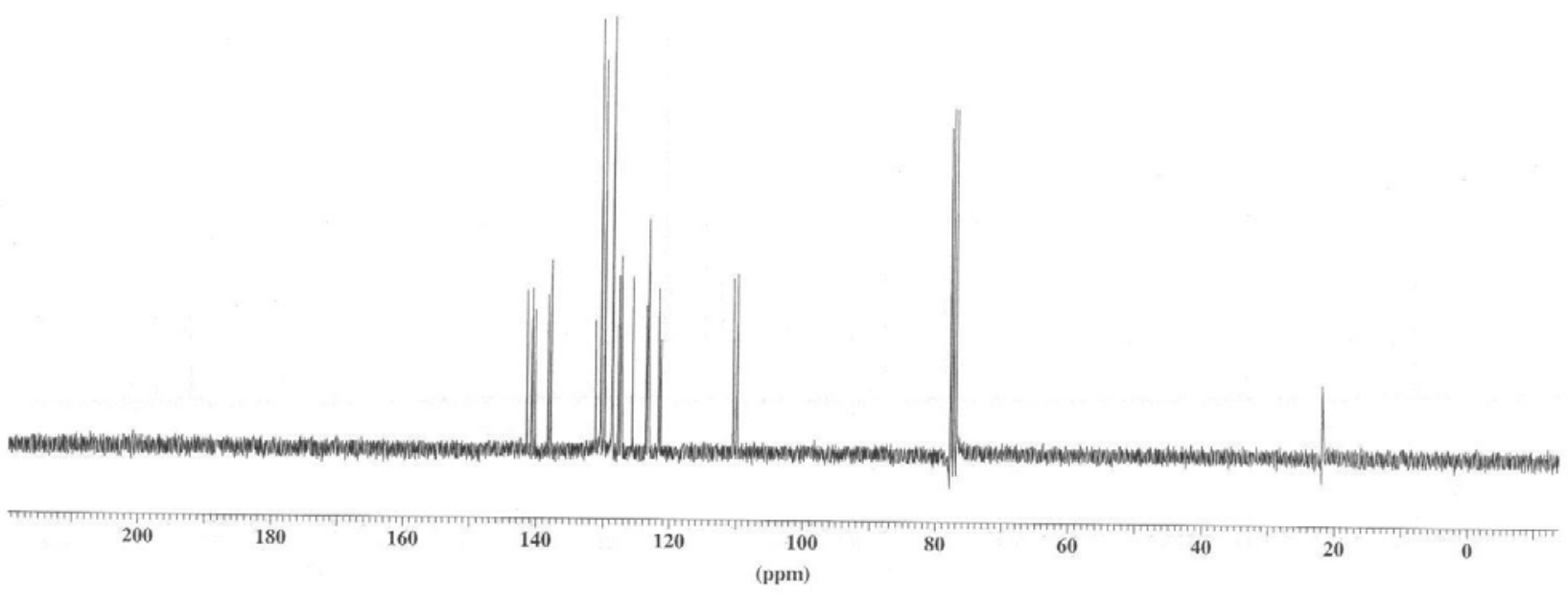


ZJ5 ZJ1

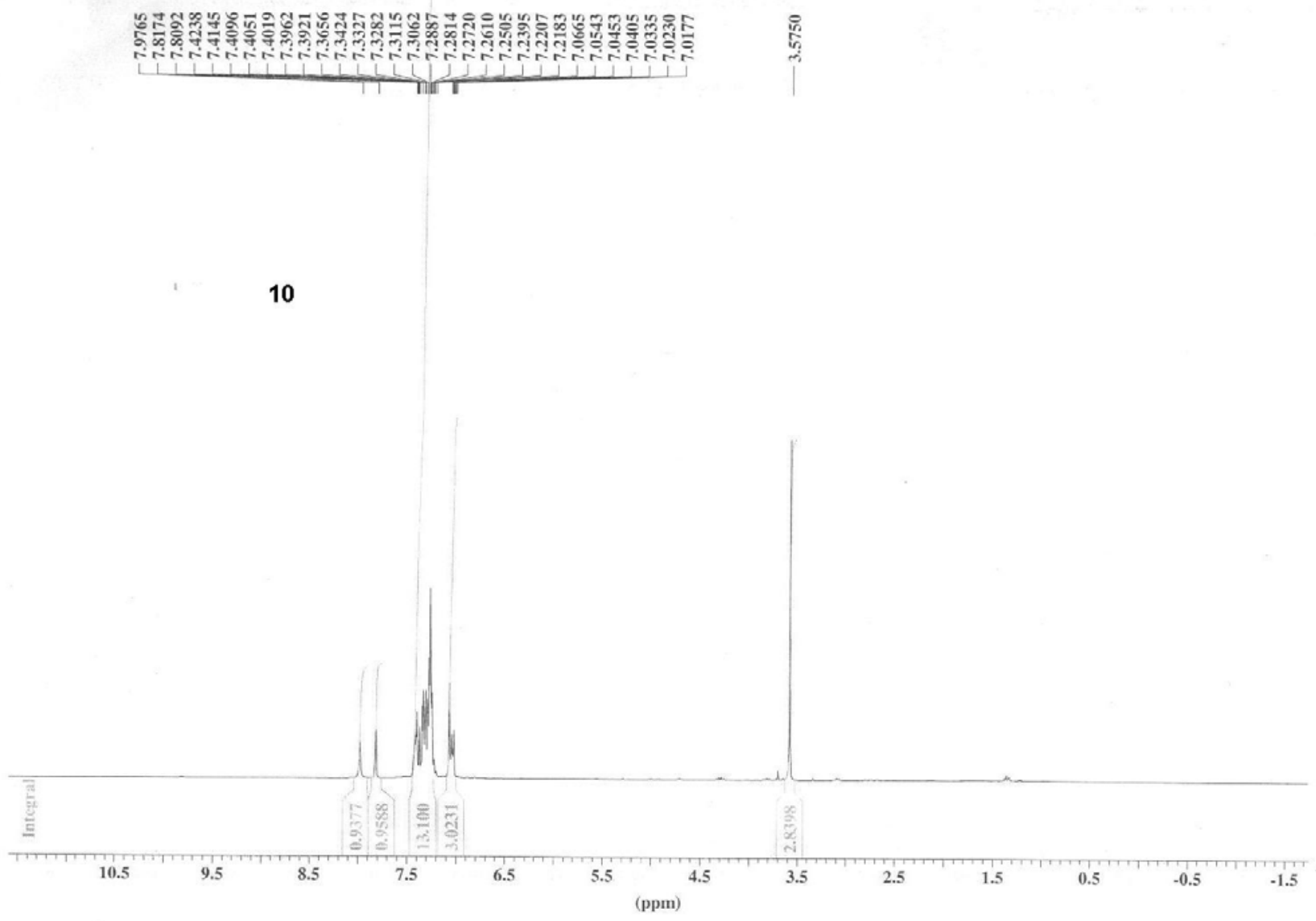




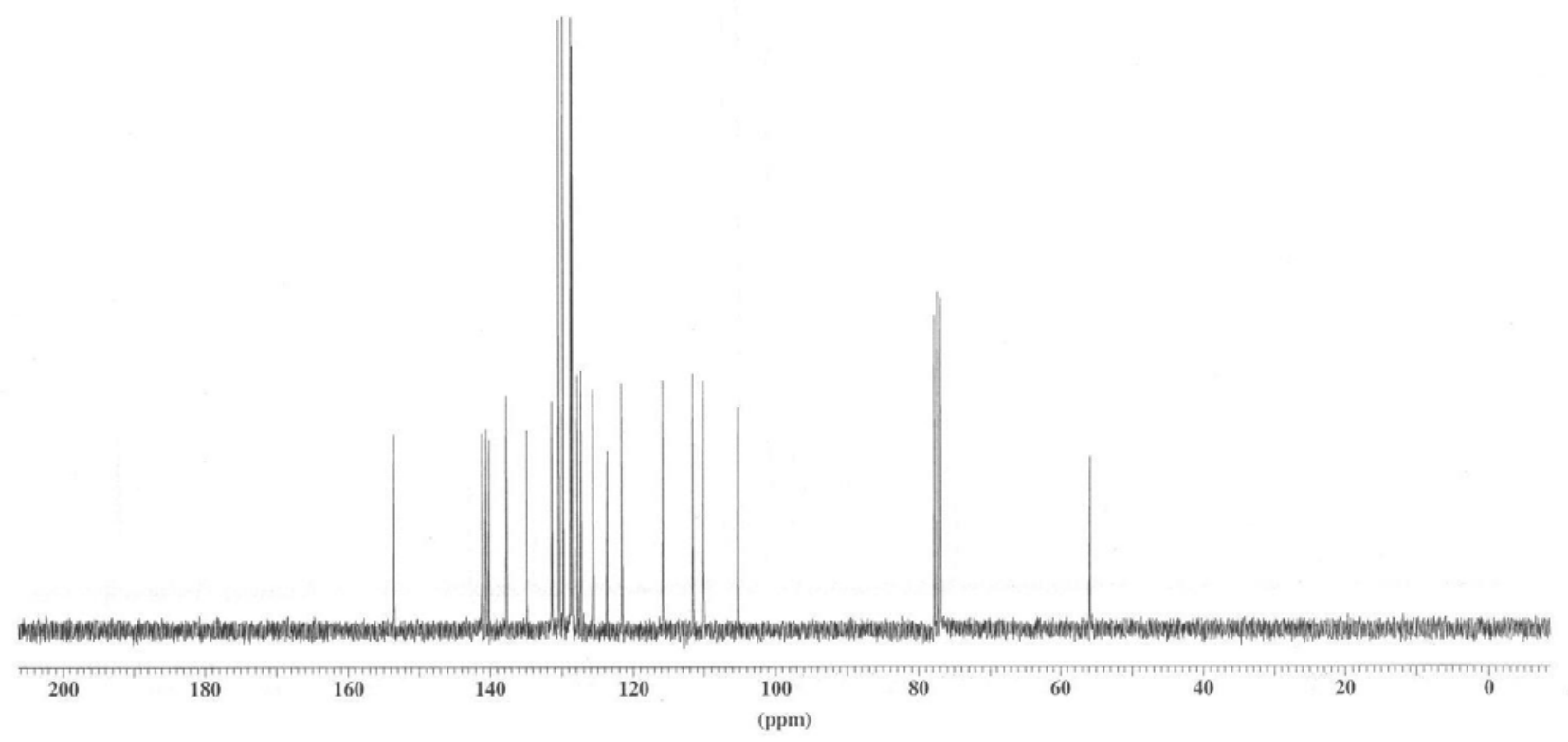




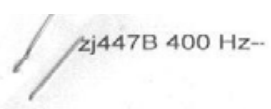

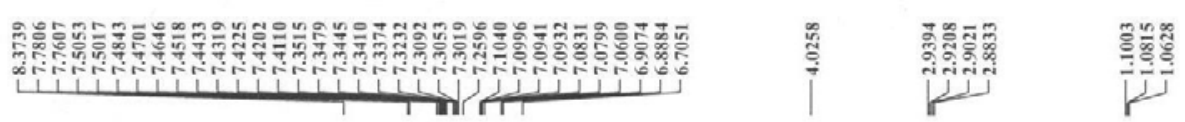

$10 a+11 b$

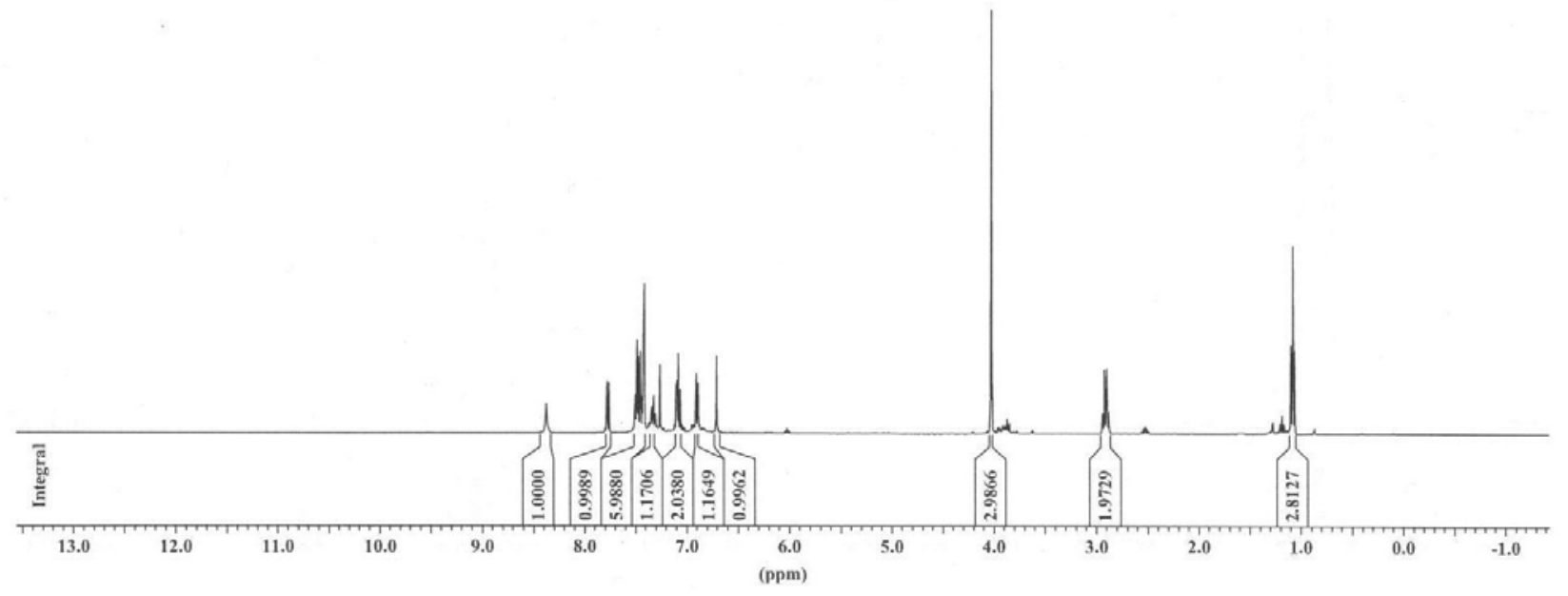


zj447b 400hz-

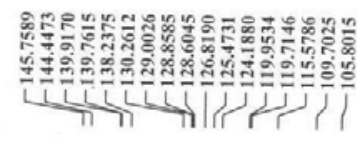

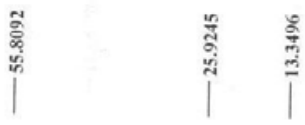

$10 a+11 b$

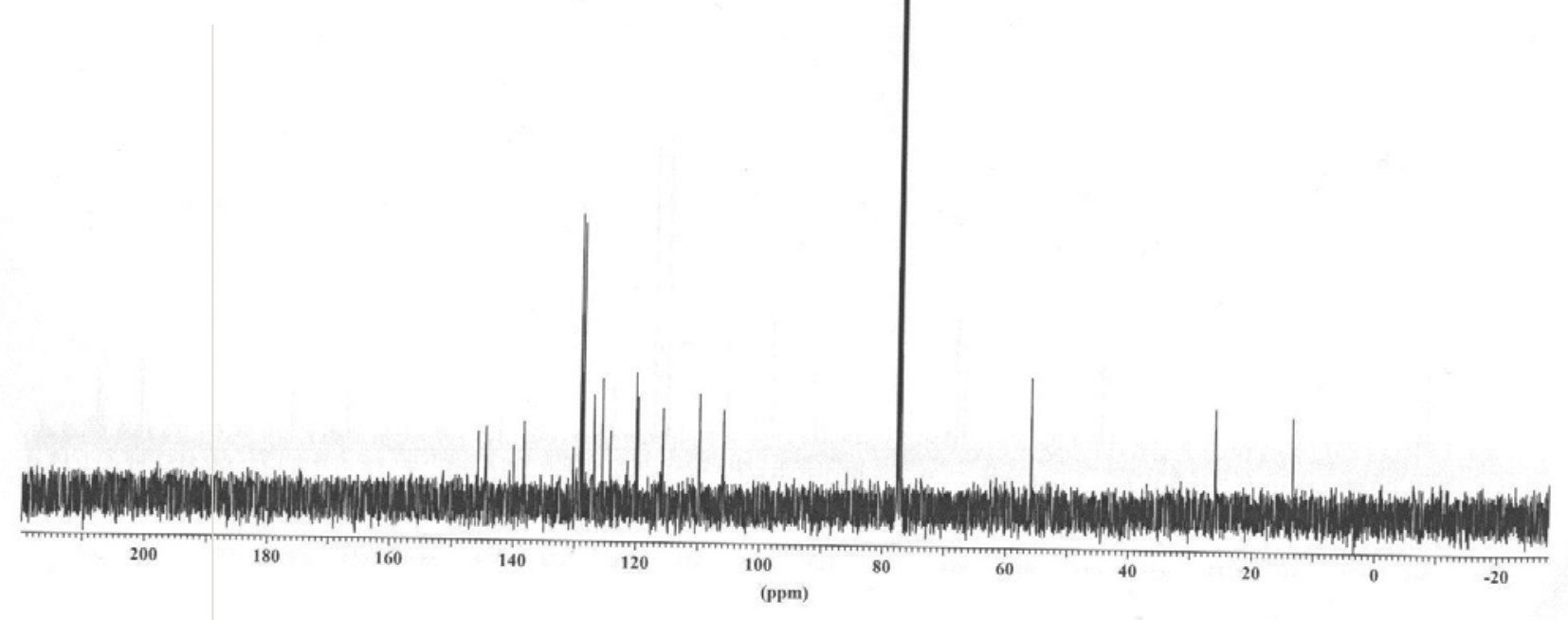



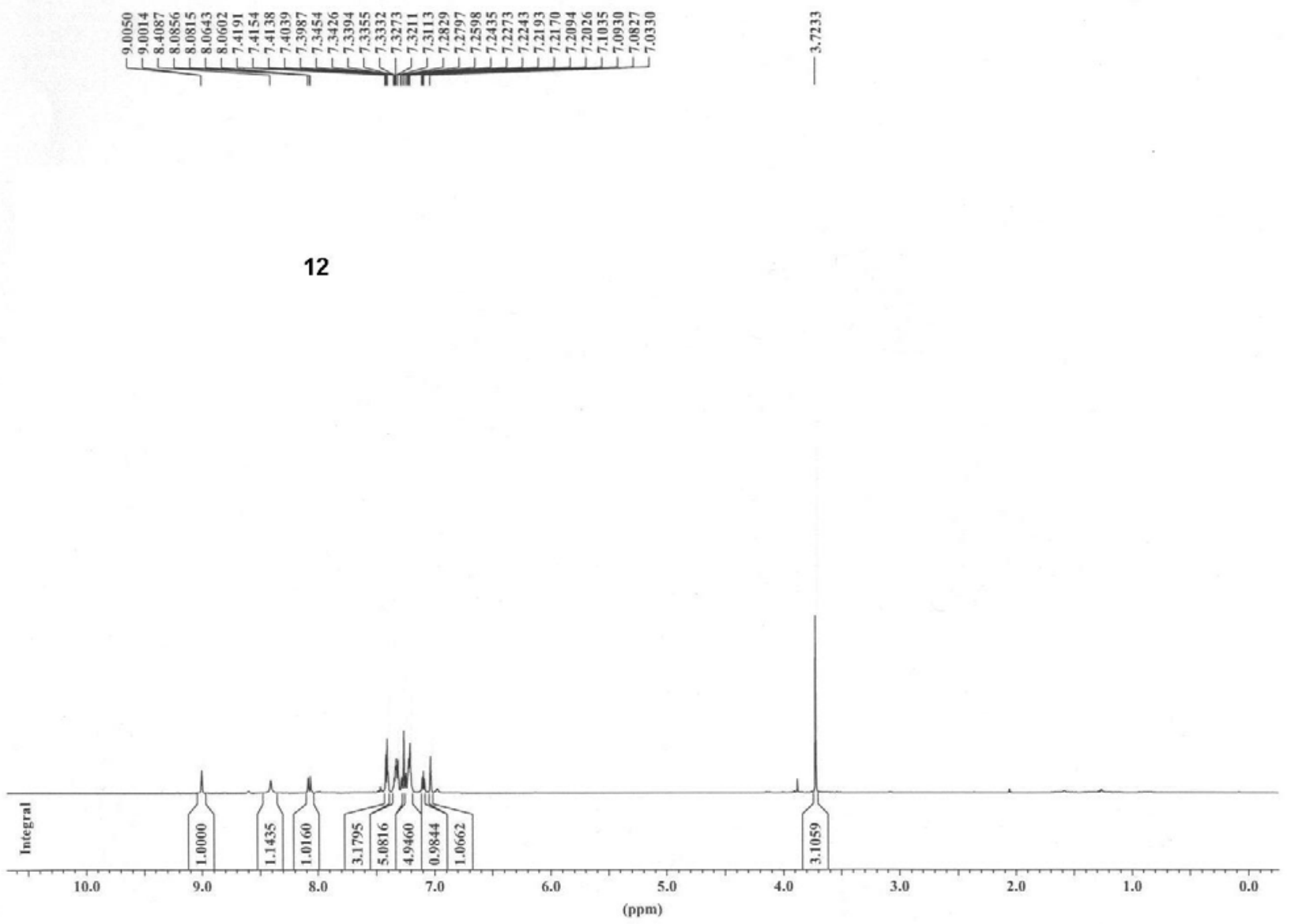


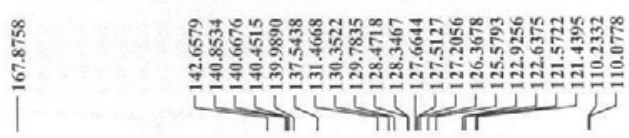

12

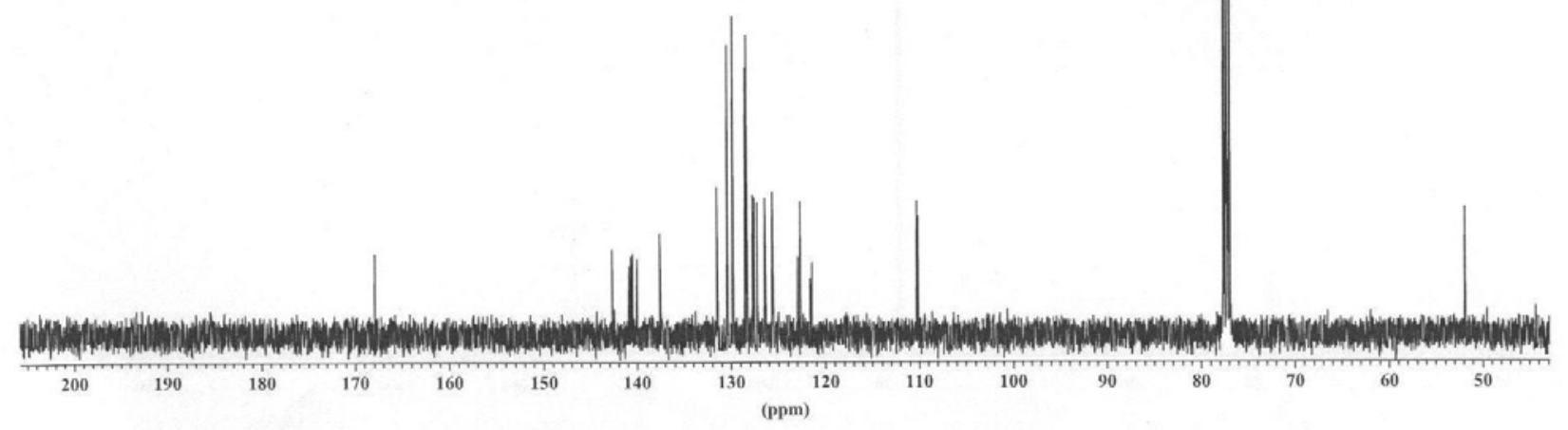


$2 J+86 B$

STANDAŔD 1 H OBSERVE

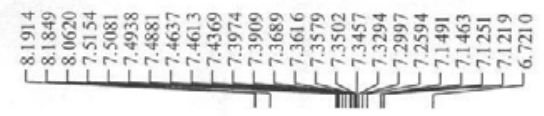

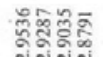

|ांग

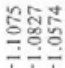

IJ

$13 a+13 b$

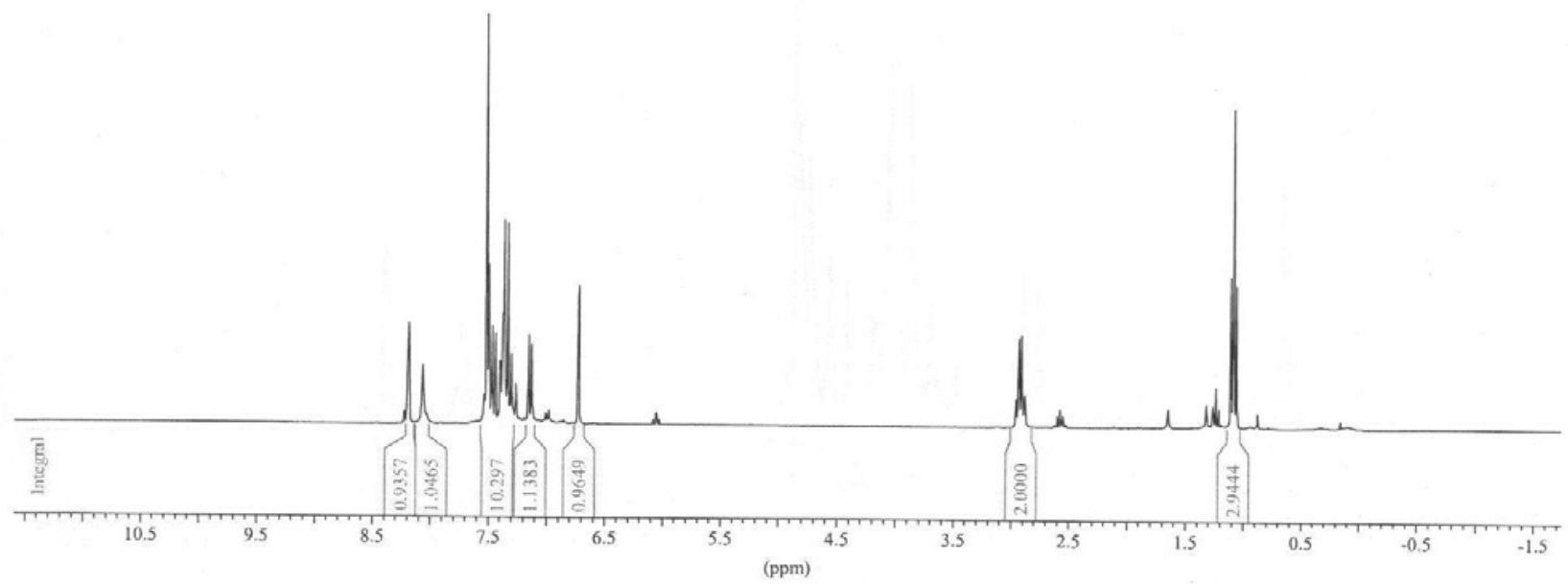




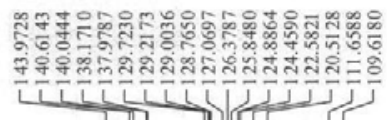

|

$13 a+13 b$

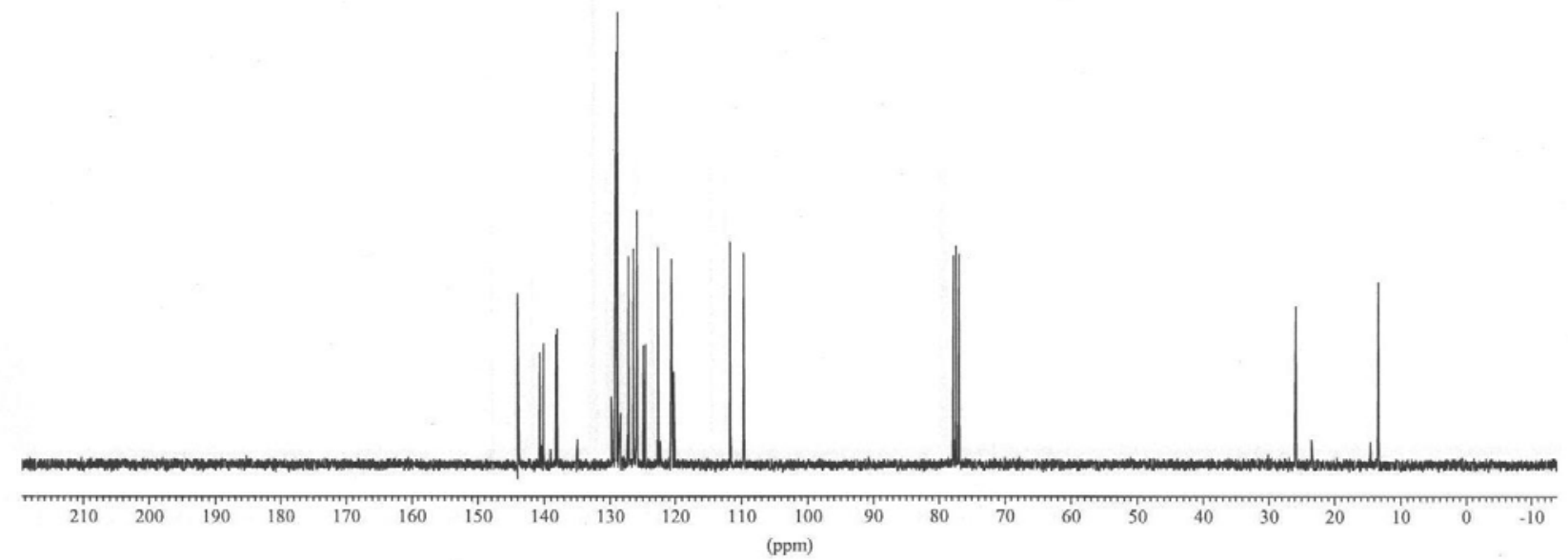



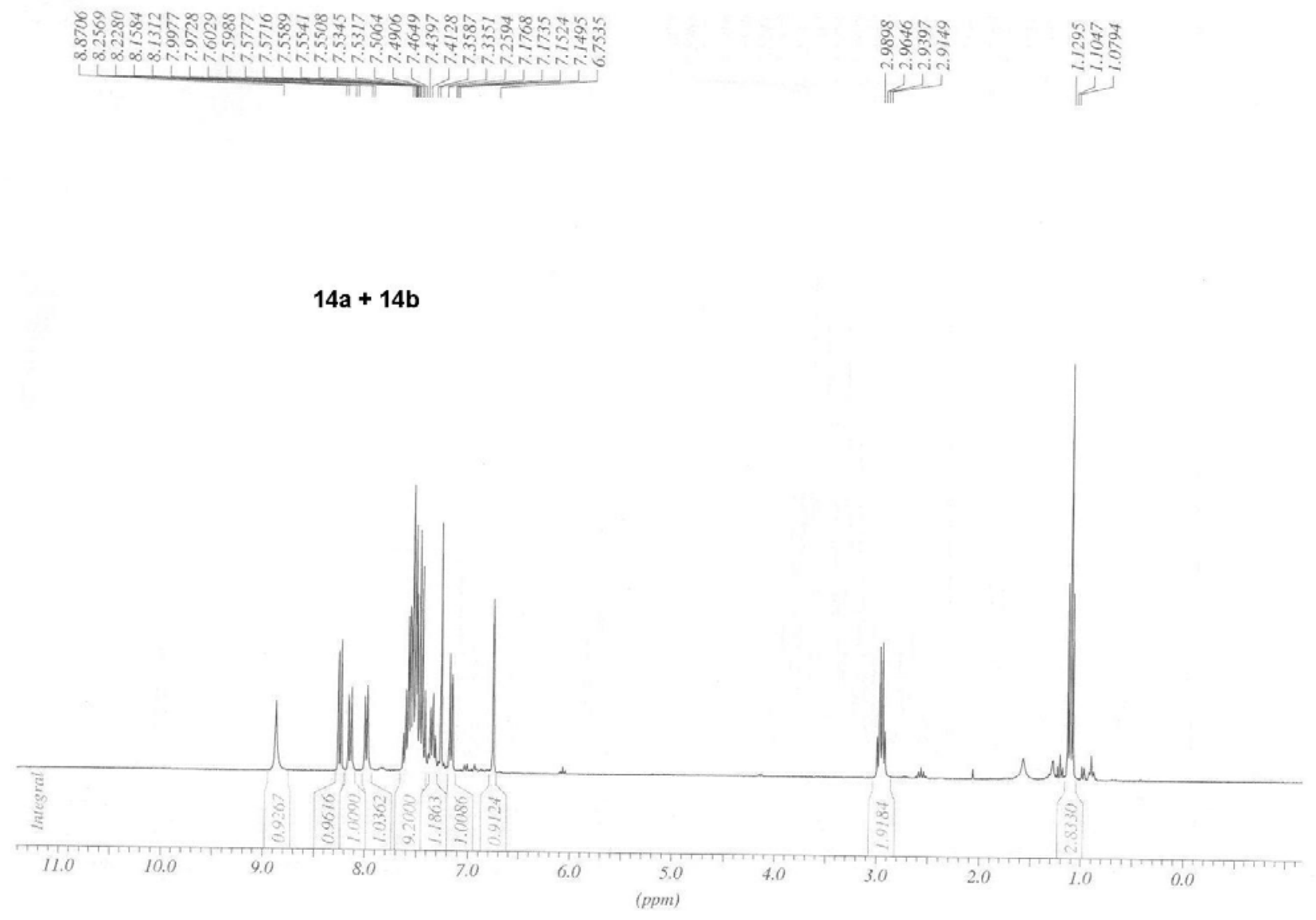


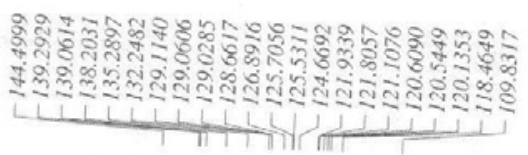

$14 a+14 b$

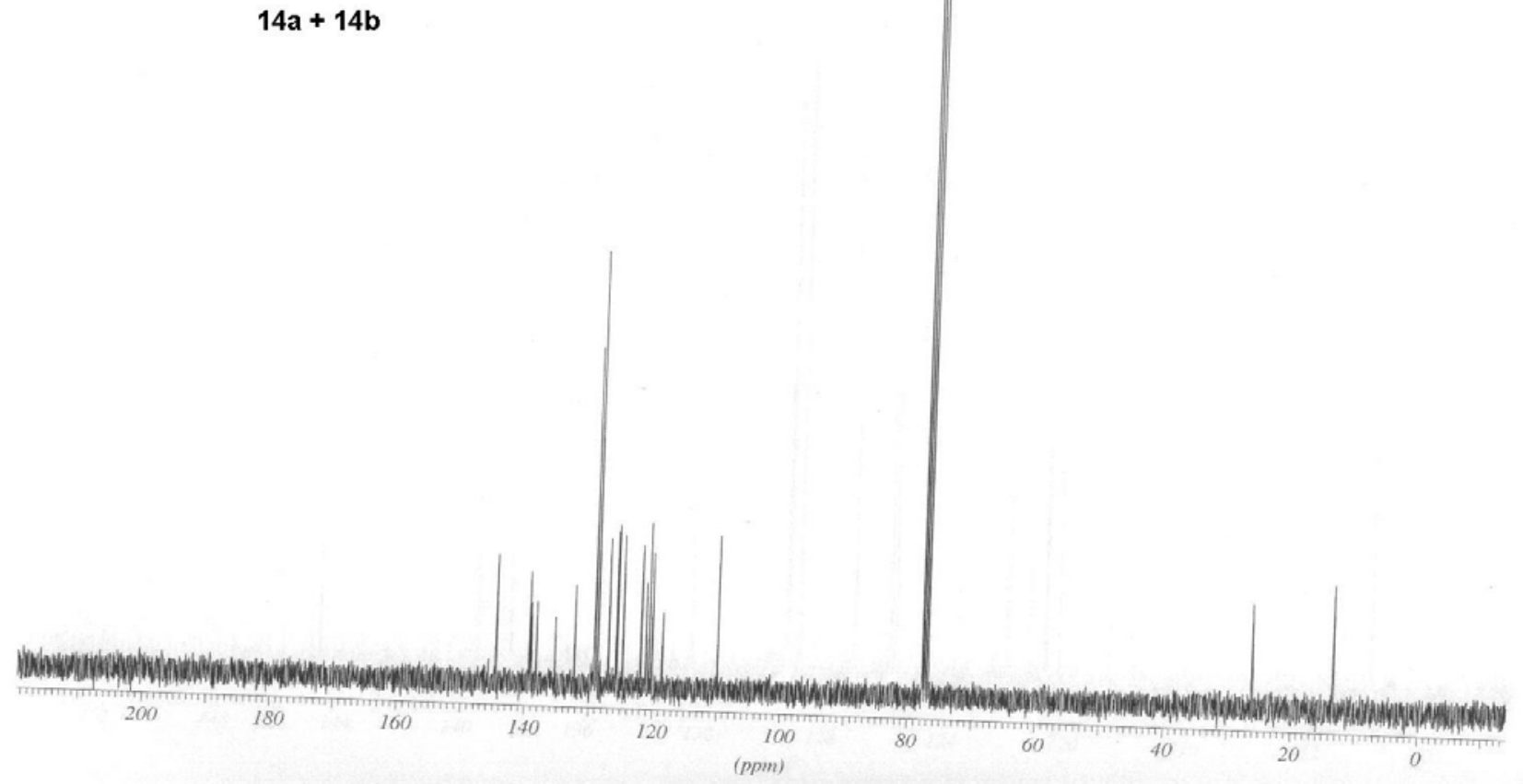


15

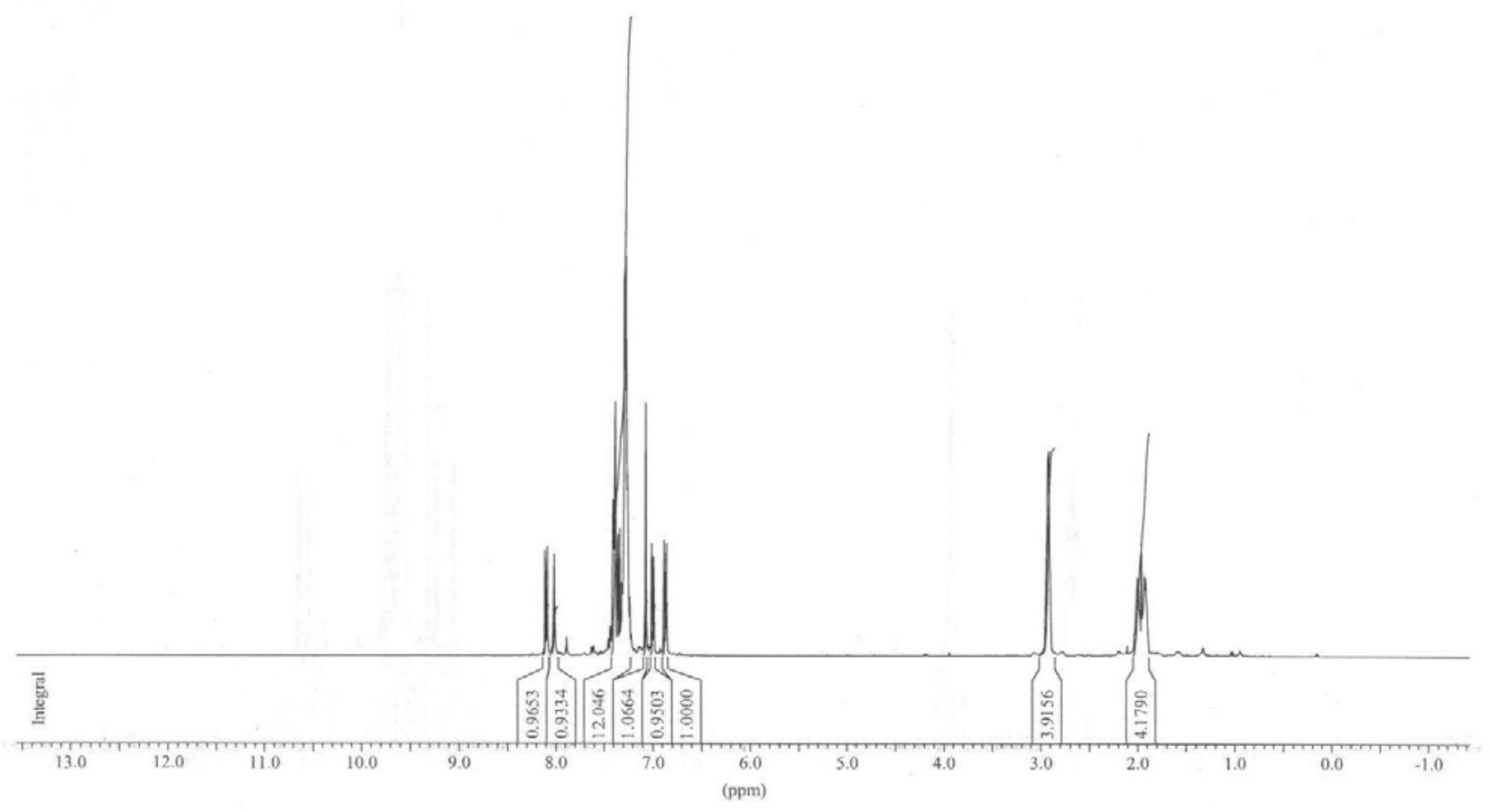


$400 \mathrm{H} 2$

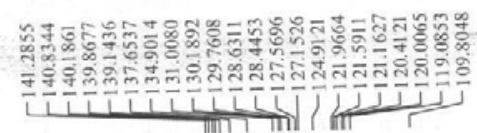

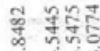

สิ สี่

1 |

15

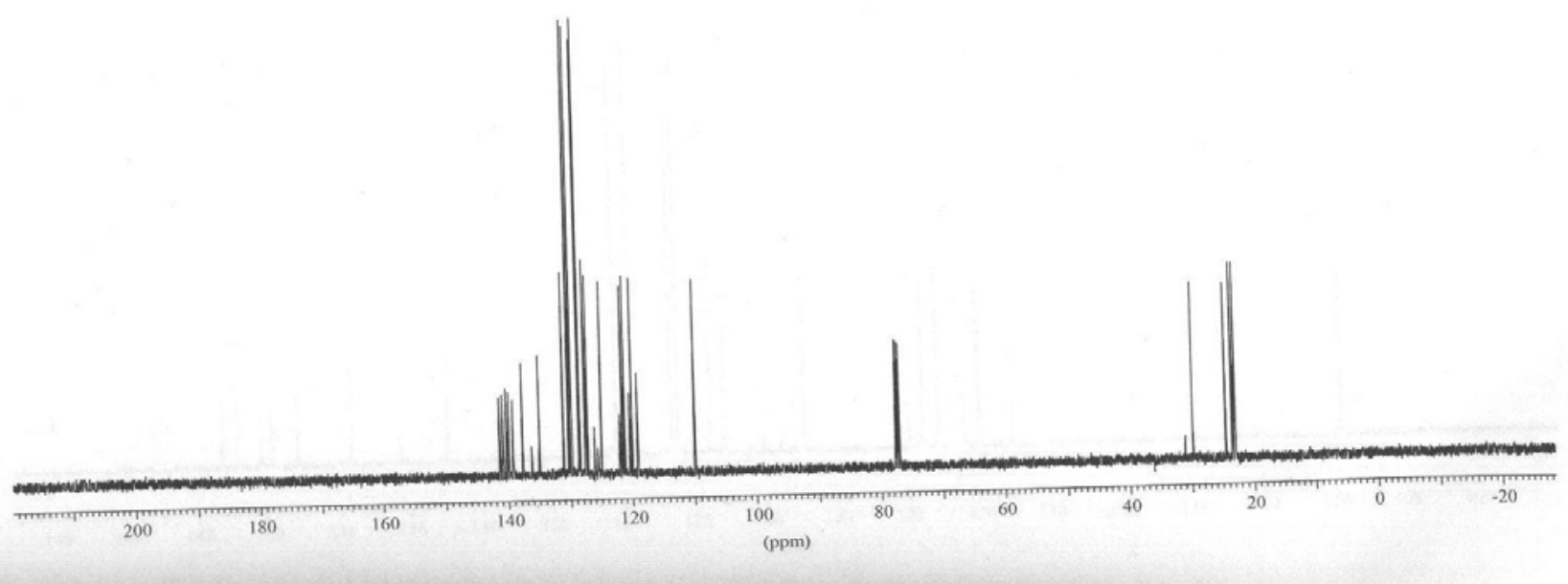


$400 \mathrm{~Hz} \quad 25338$

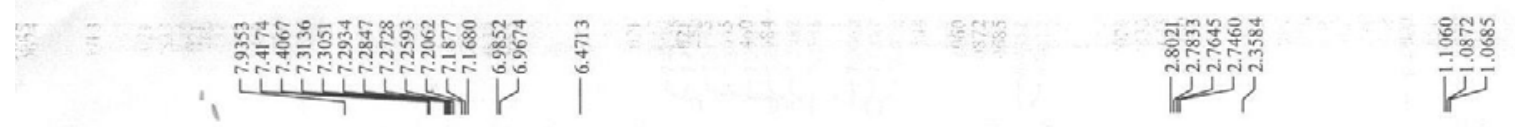

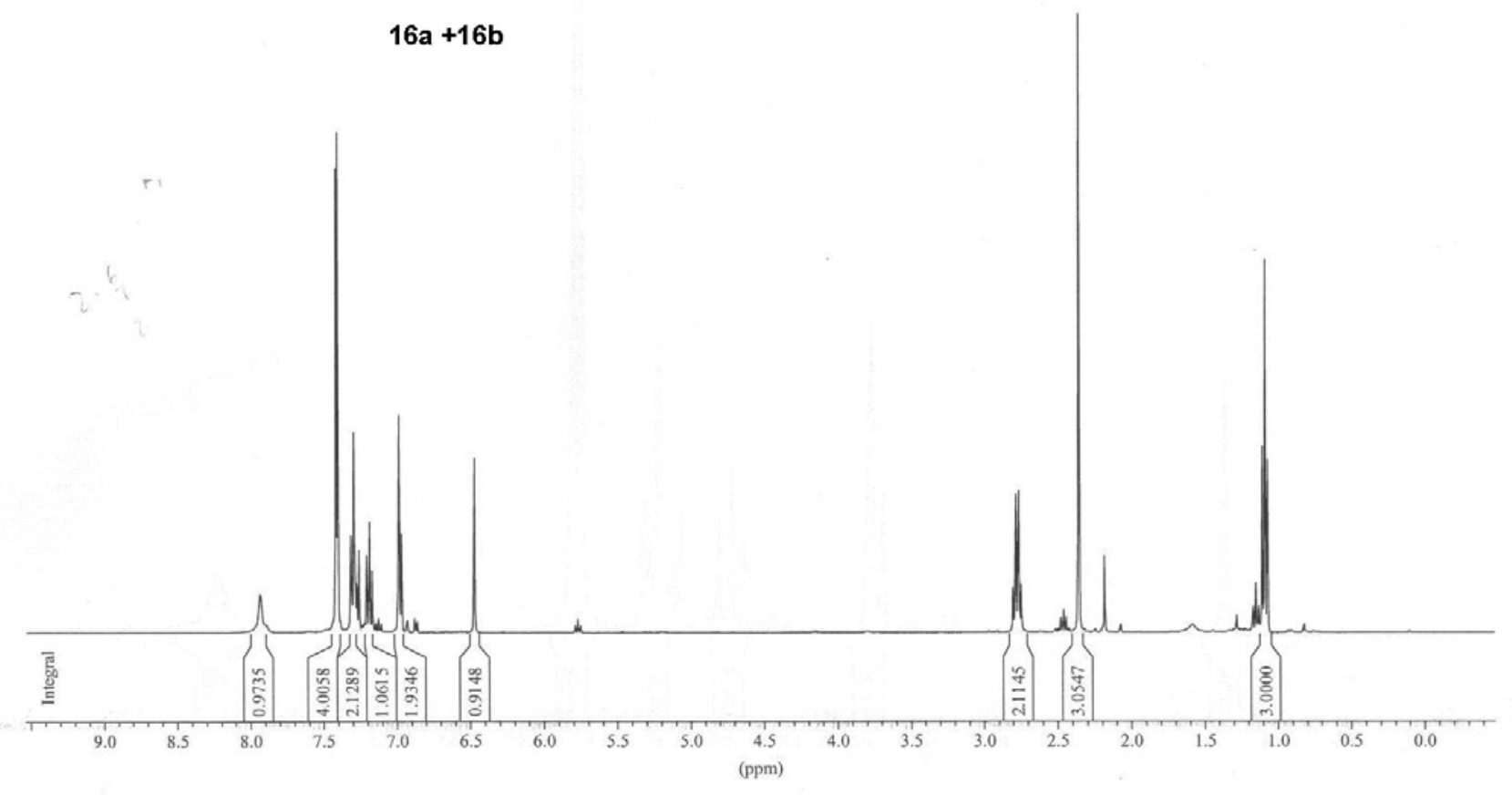




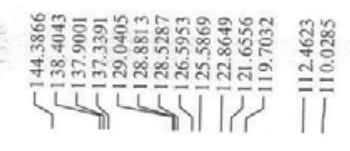

i

$16 a+16 b$

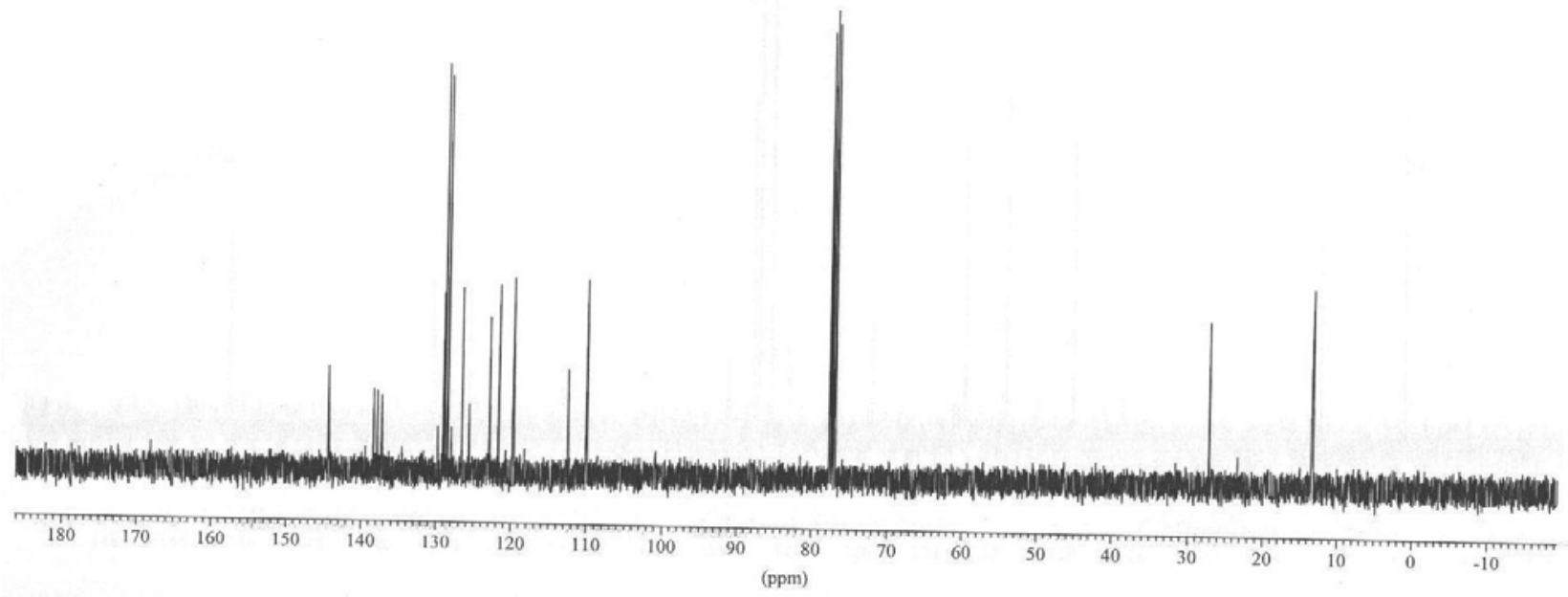




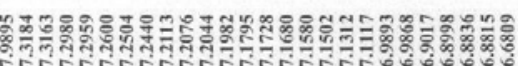

iाtा $\frac{1}{1}$

17

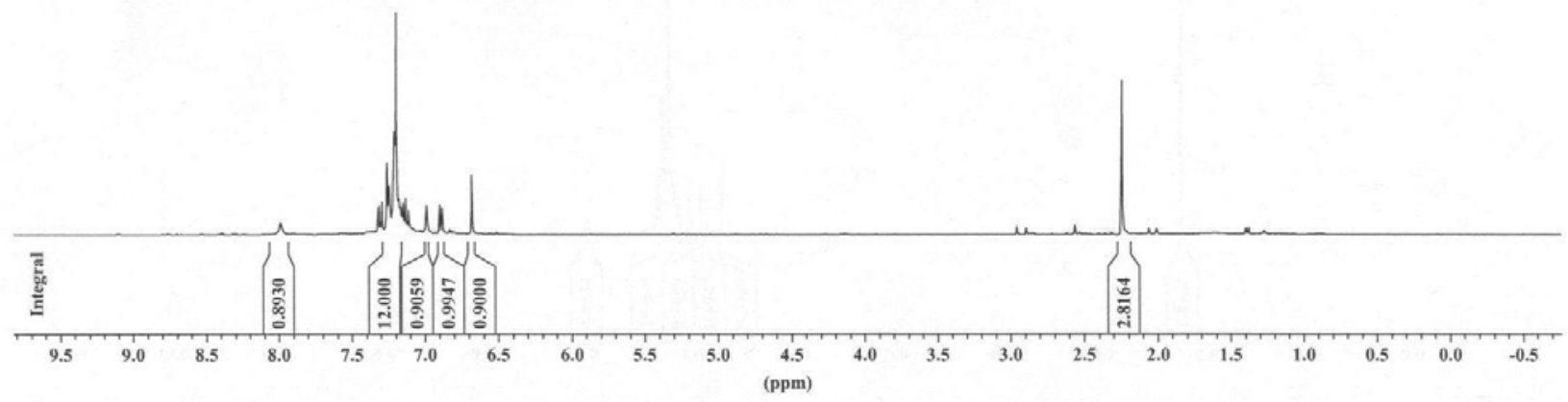




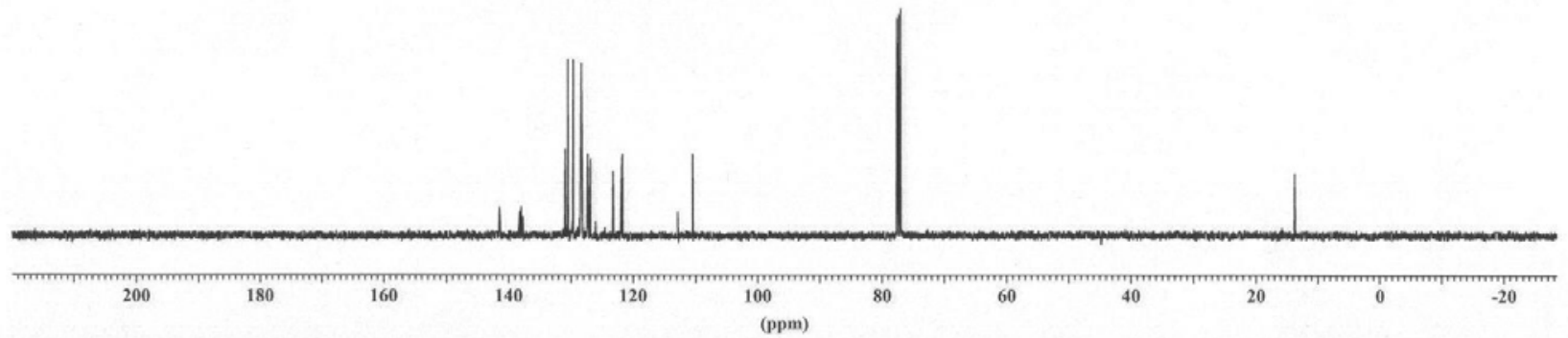


$2 \mathrm{~J} 850400 \mathrm{~Hz}-$

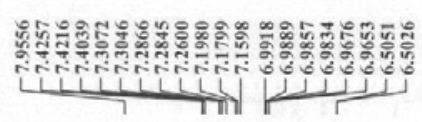

$18 a+18 b$

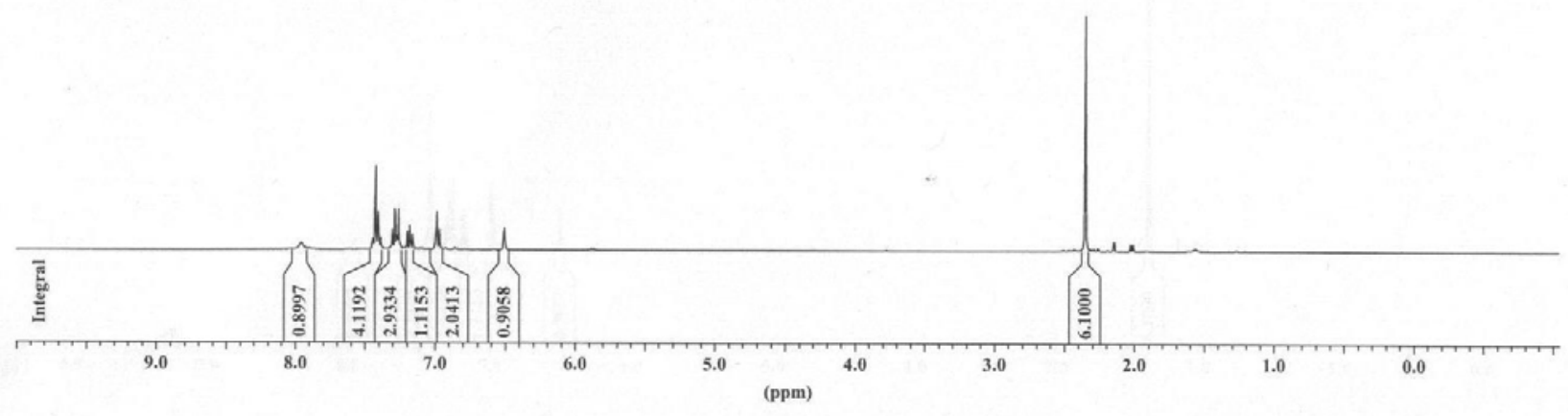




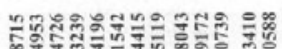

0.ं.

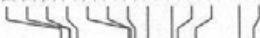

|

$18 a+18 b$

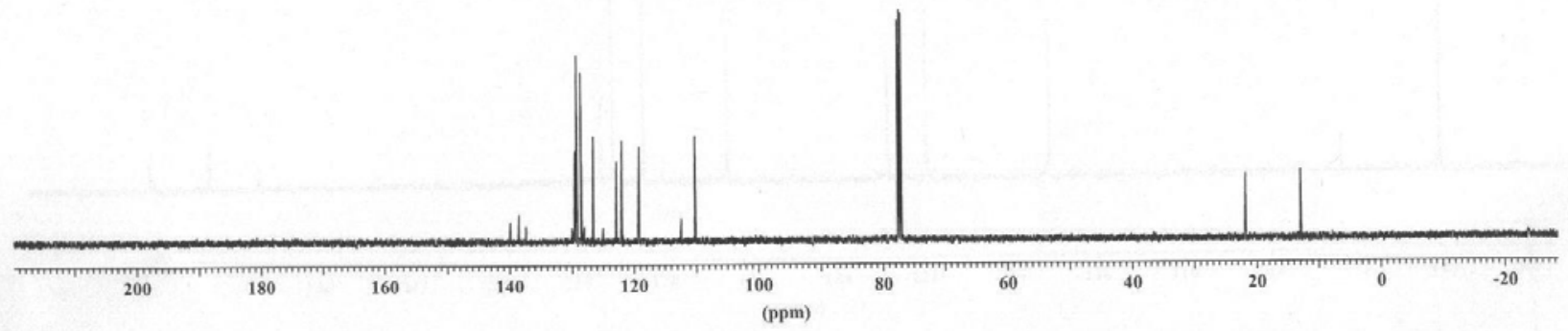




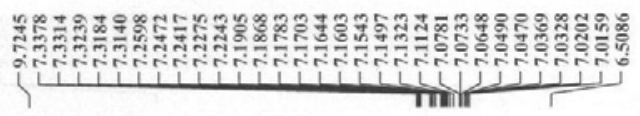

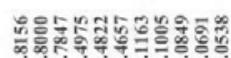

4 1

19

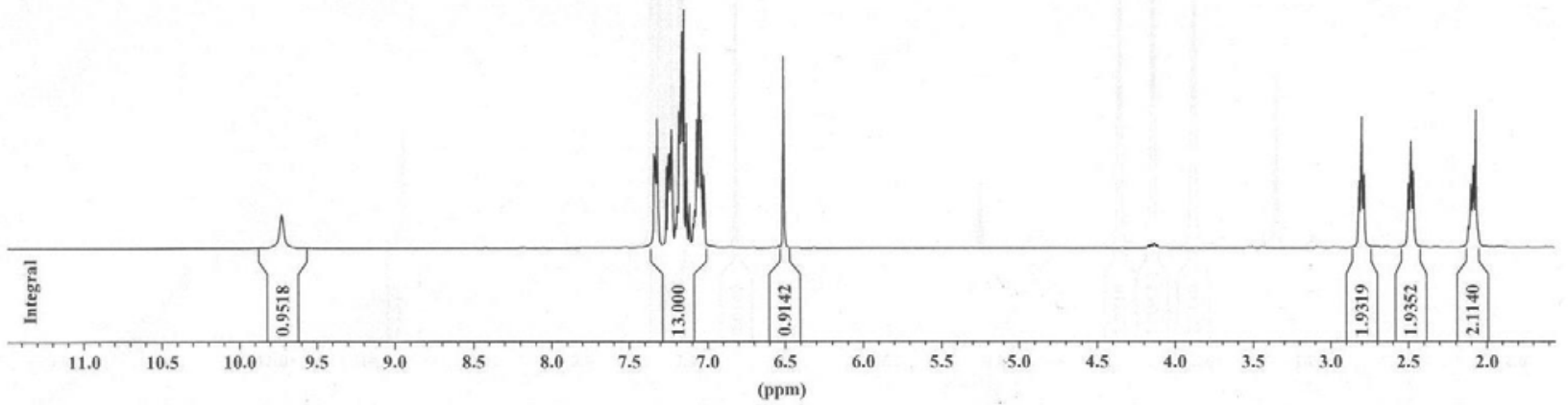




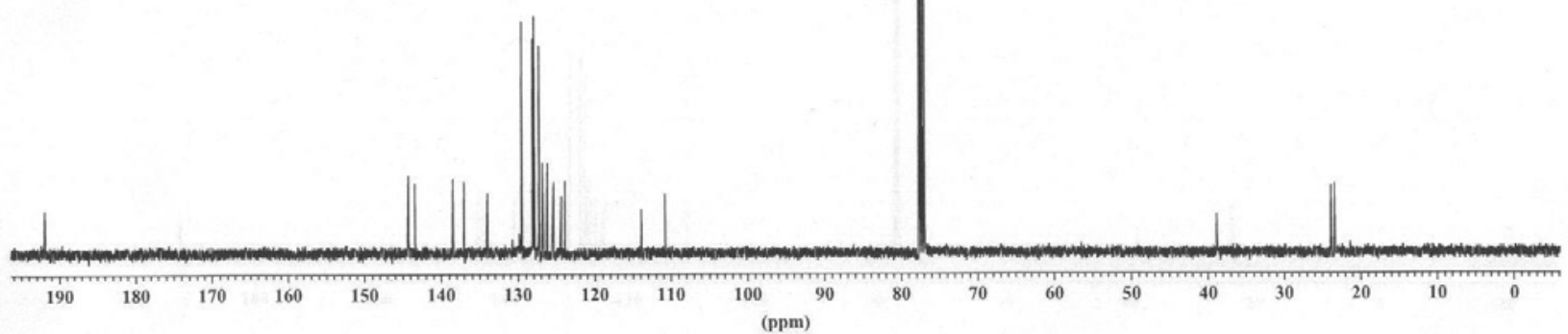



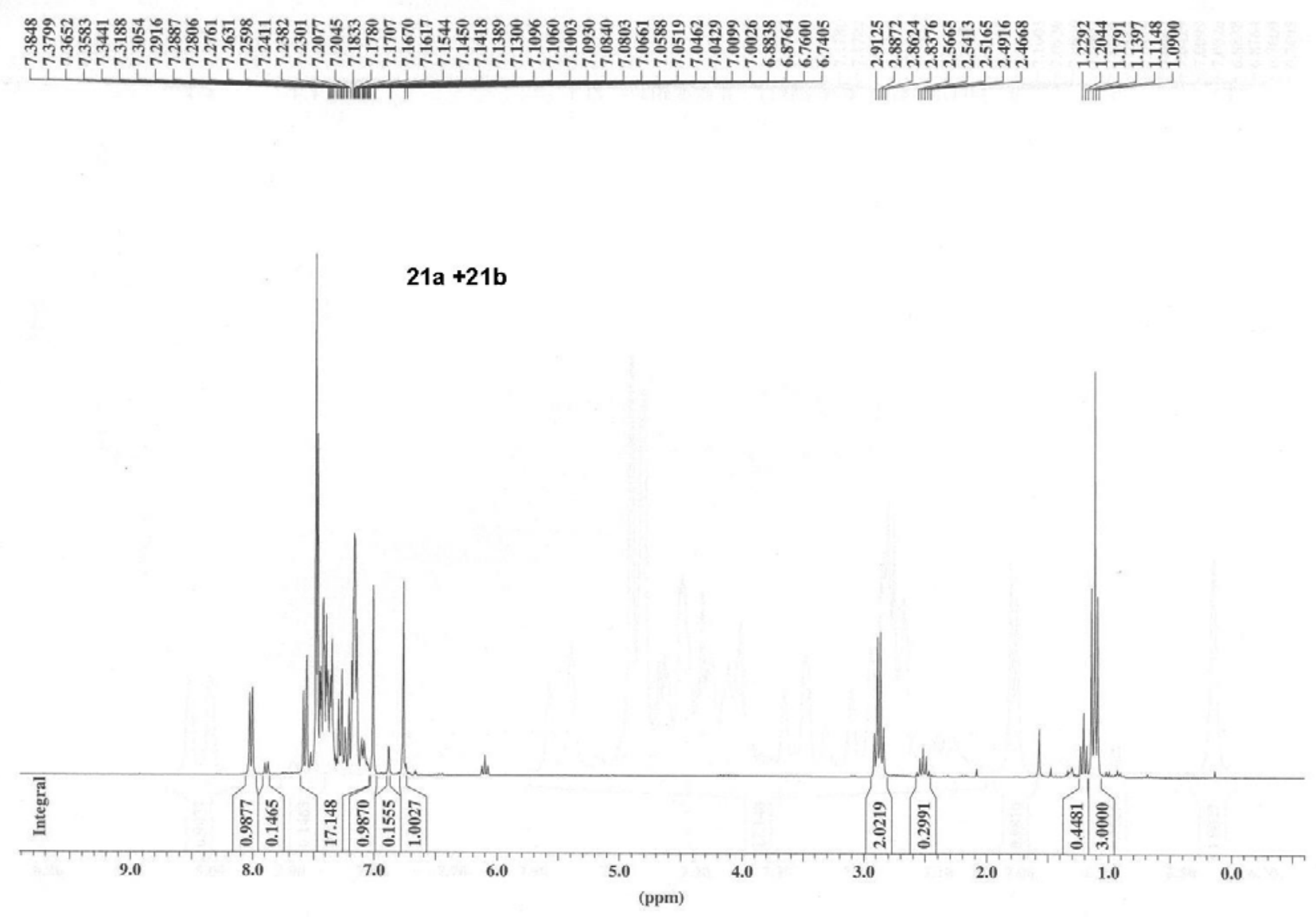


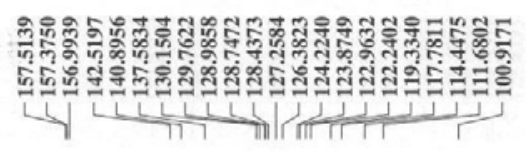

वे लै

$21 a+21 b$

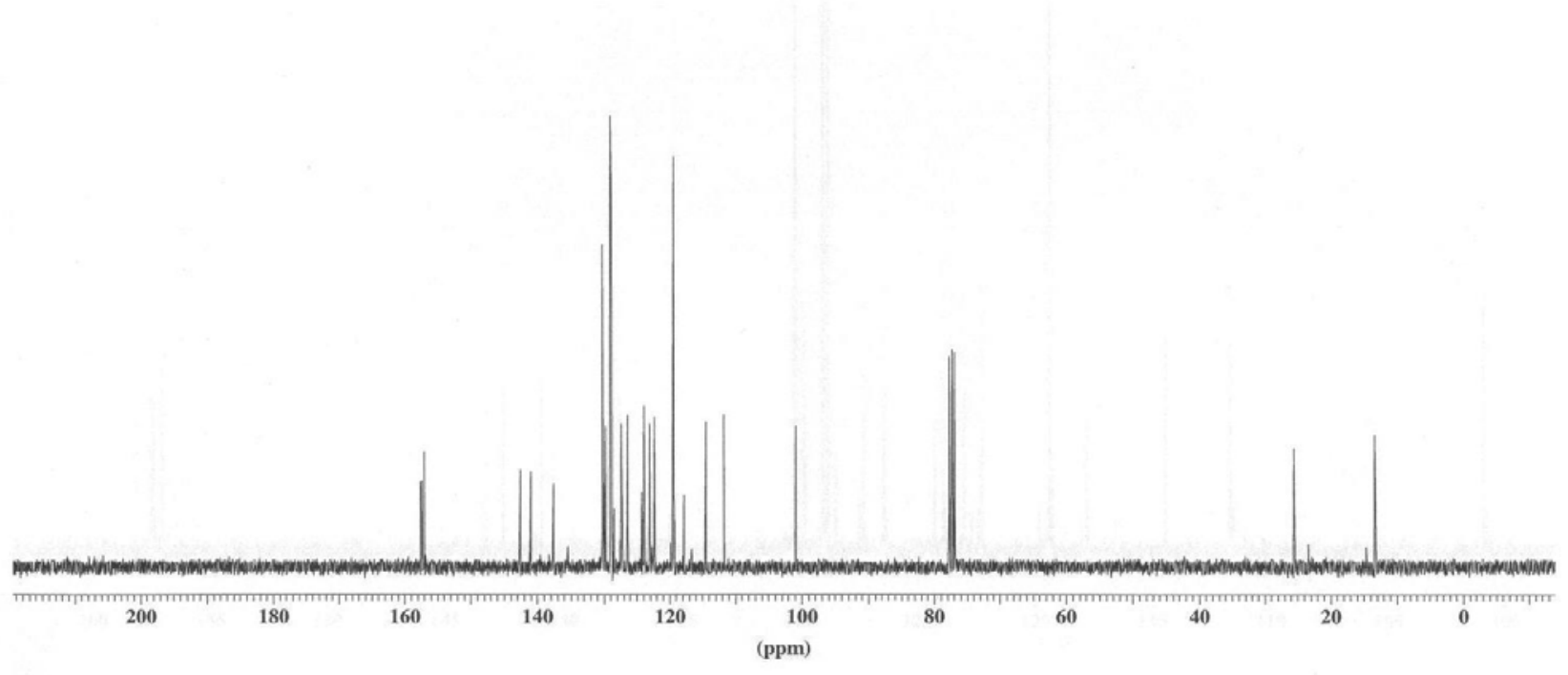


/ STANDARD 1H OBSERVE

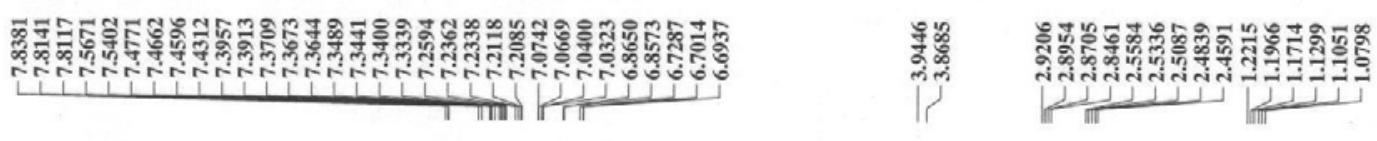

$22 a+22 b$

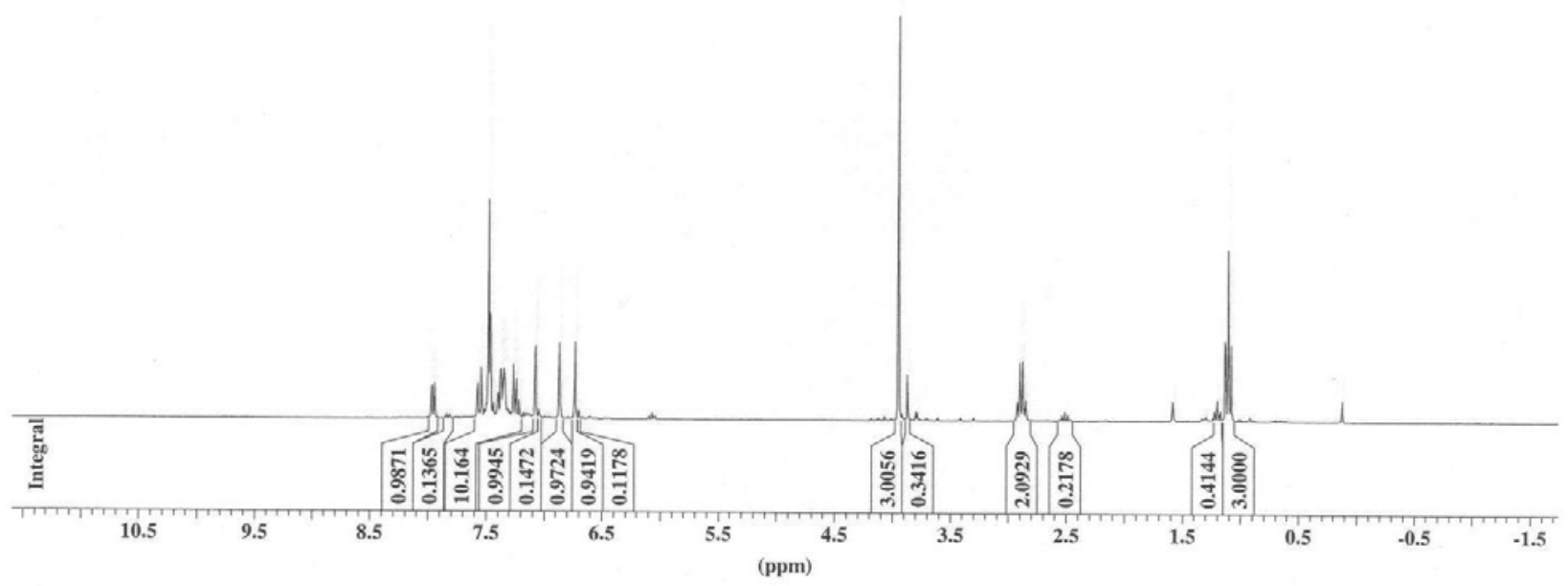




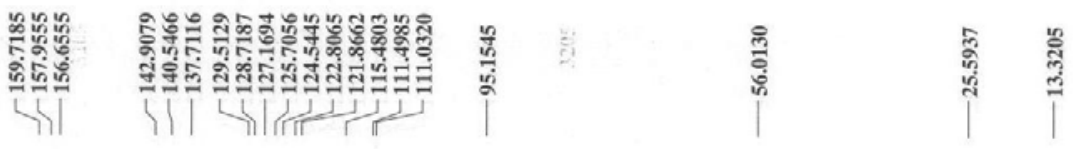

$22 a+22 b$

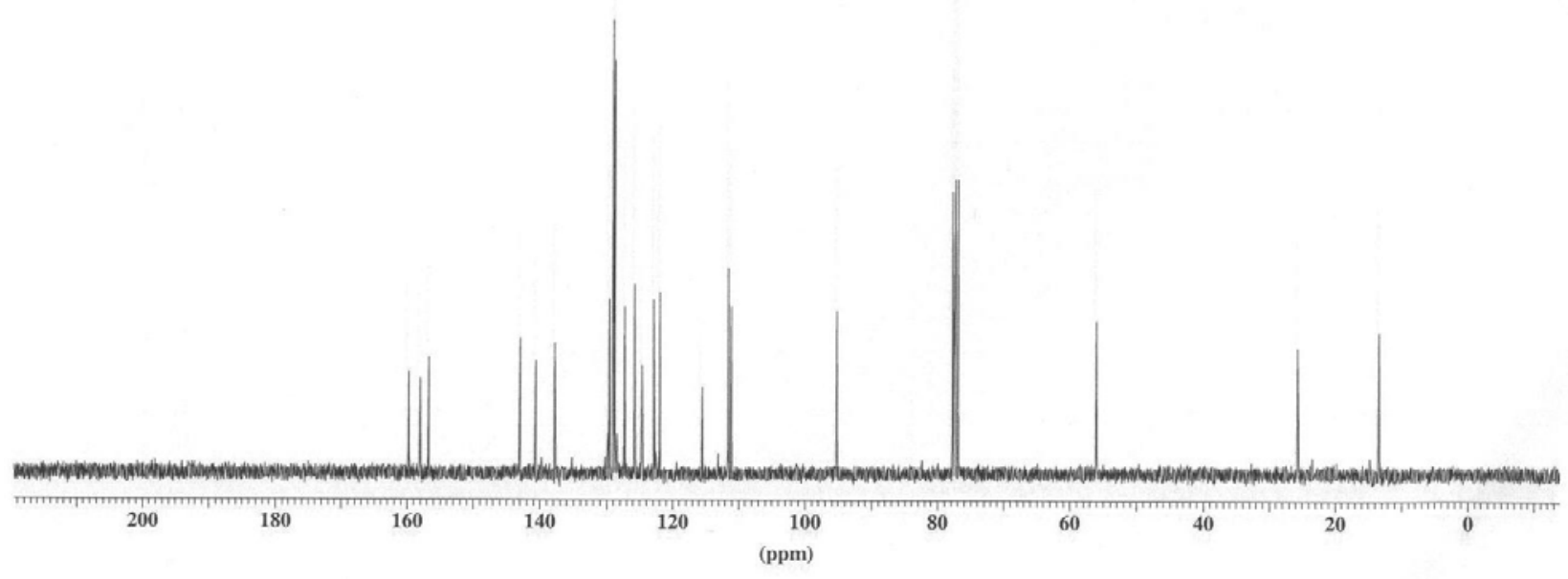


UDb

STANDARD $1 H$ OBSERVE

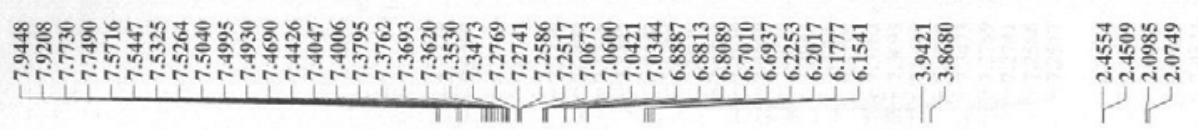

$23 a+23 b$

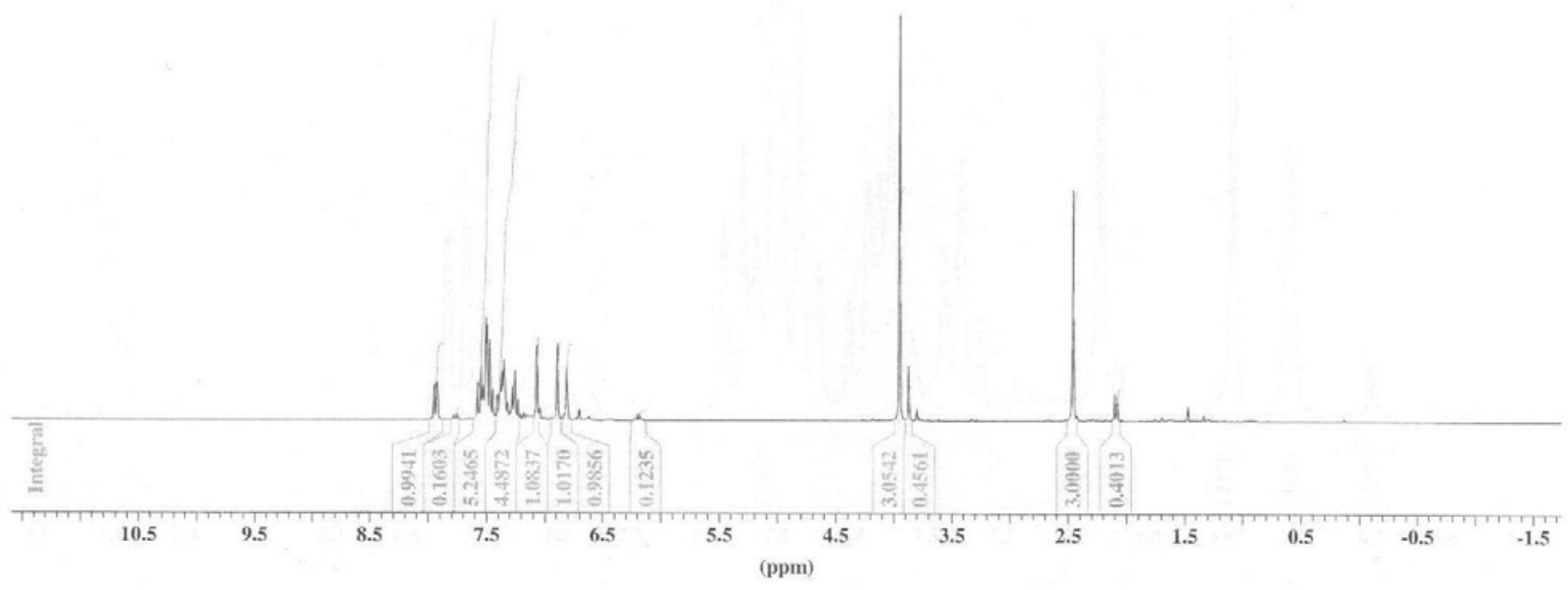




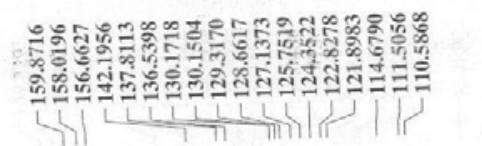

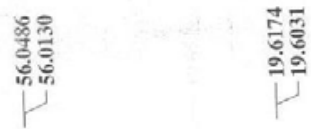

$23 a+23 b$

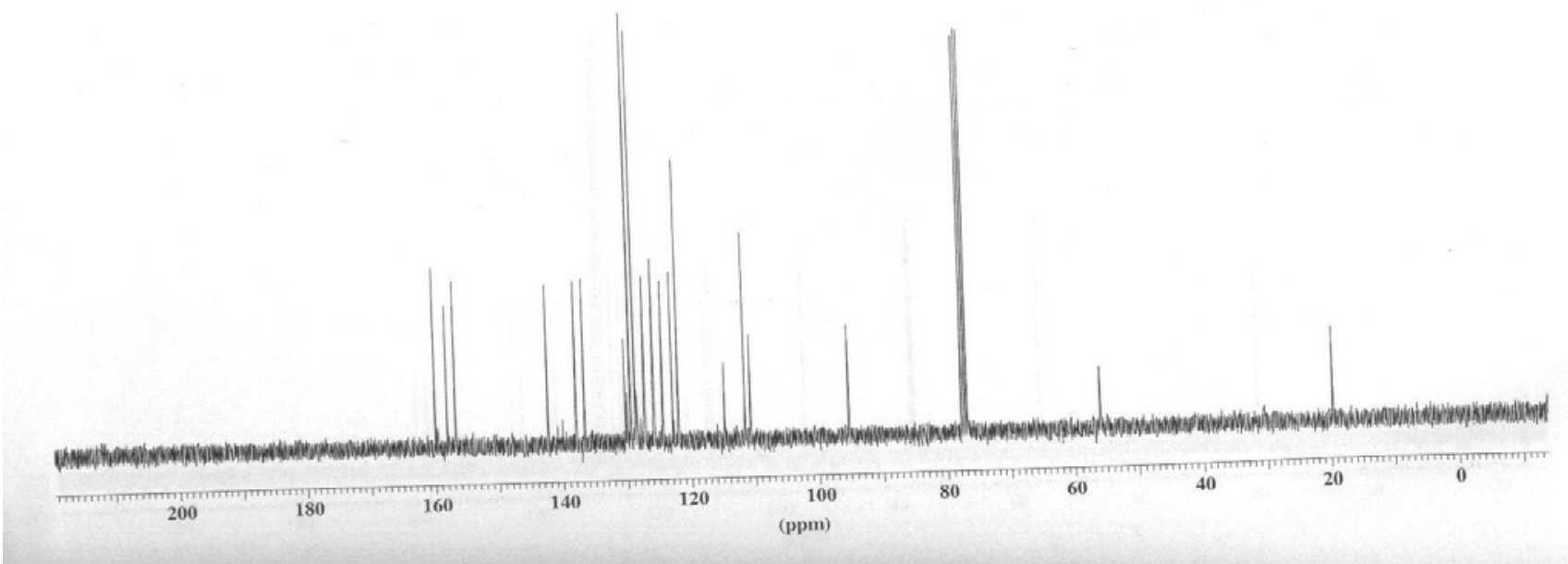



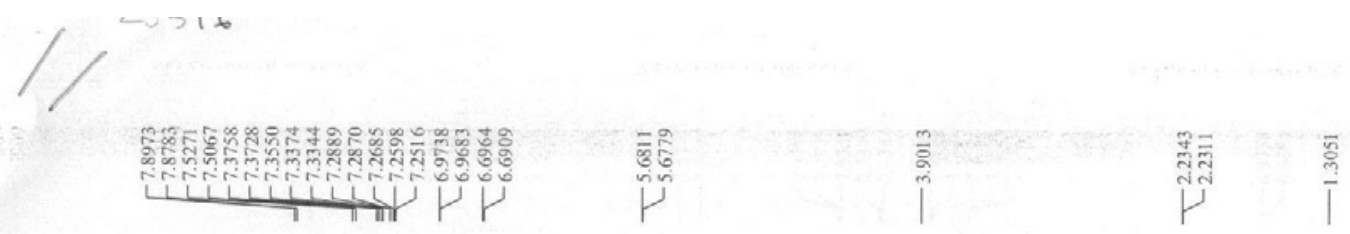

$24 a$

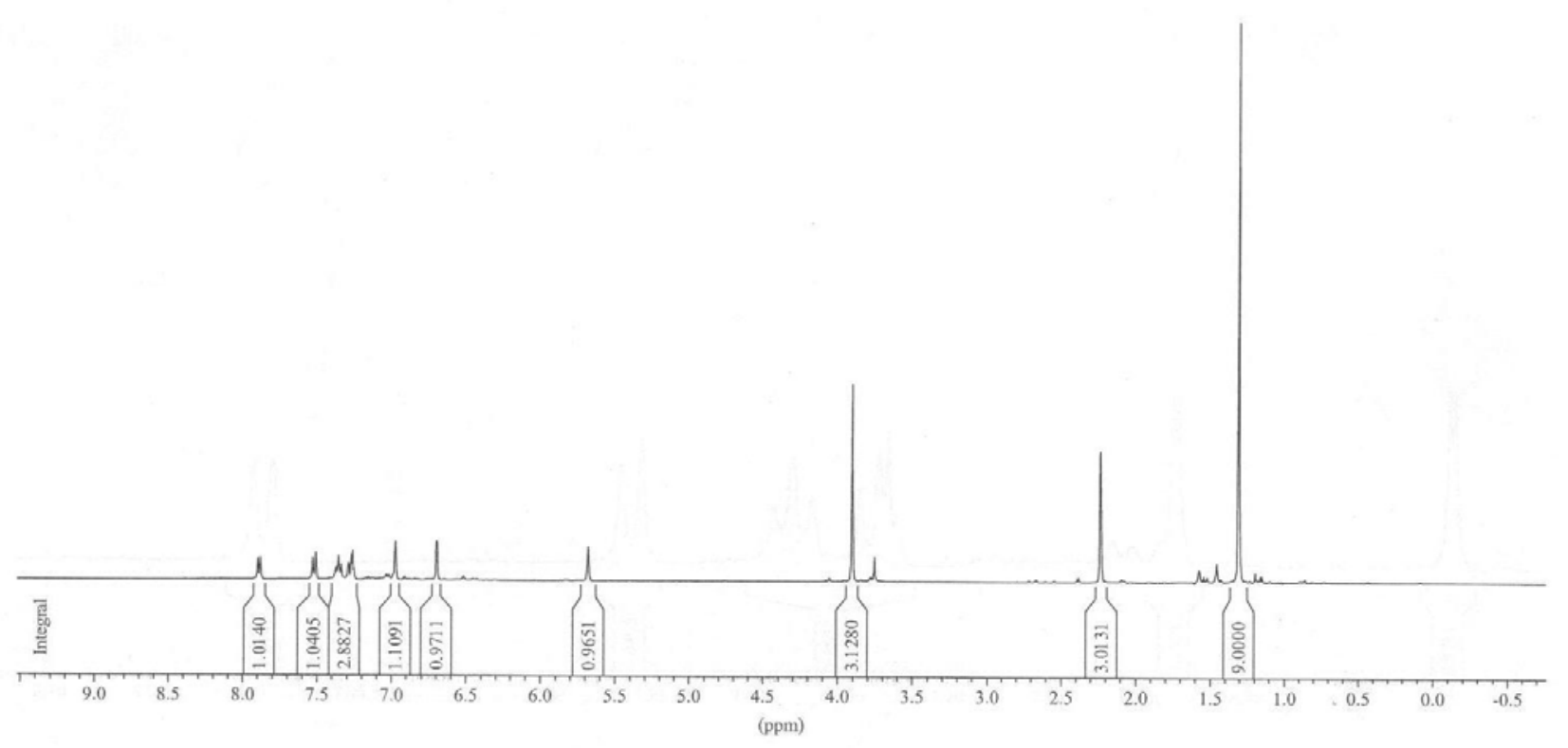




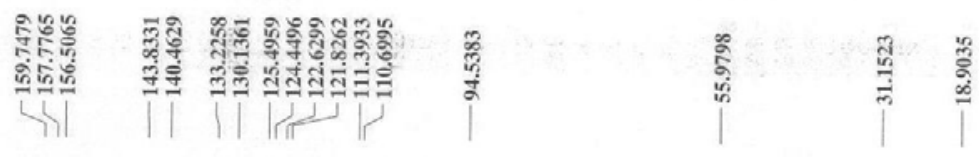

$24 a$

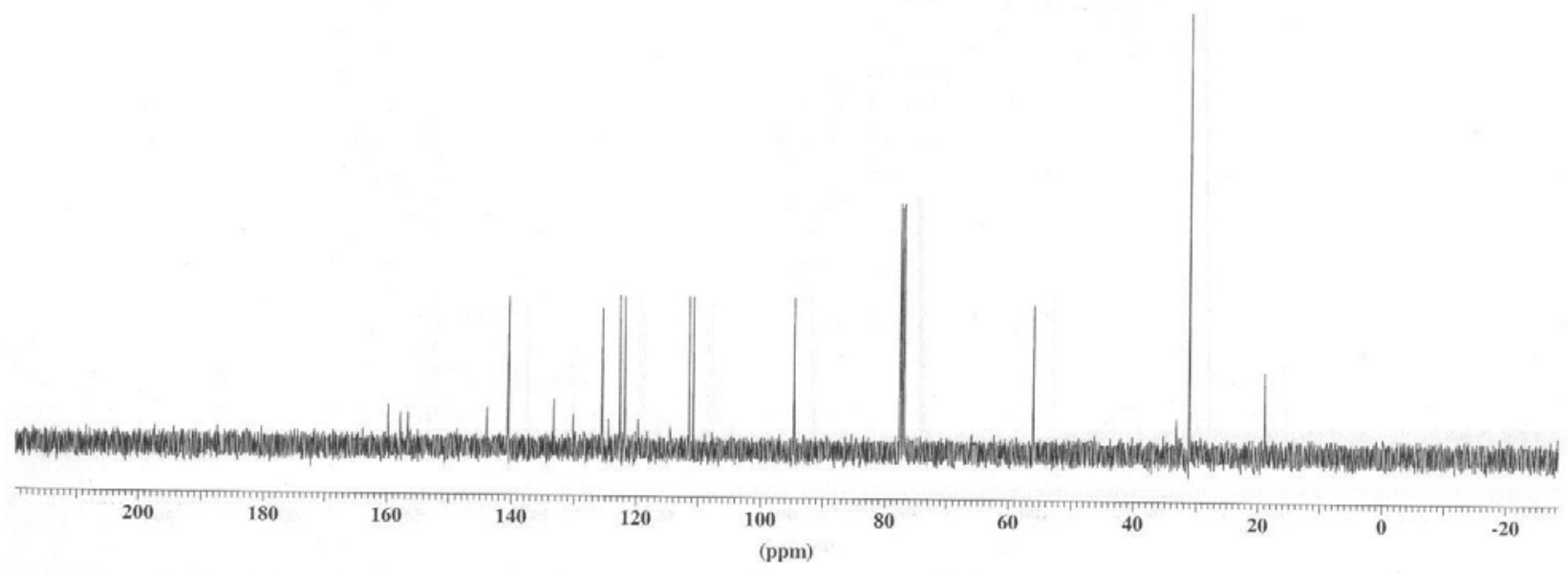


$\int$ grANDARD HH OBSERVE $_{2} \mathrm{JI}_{1}$
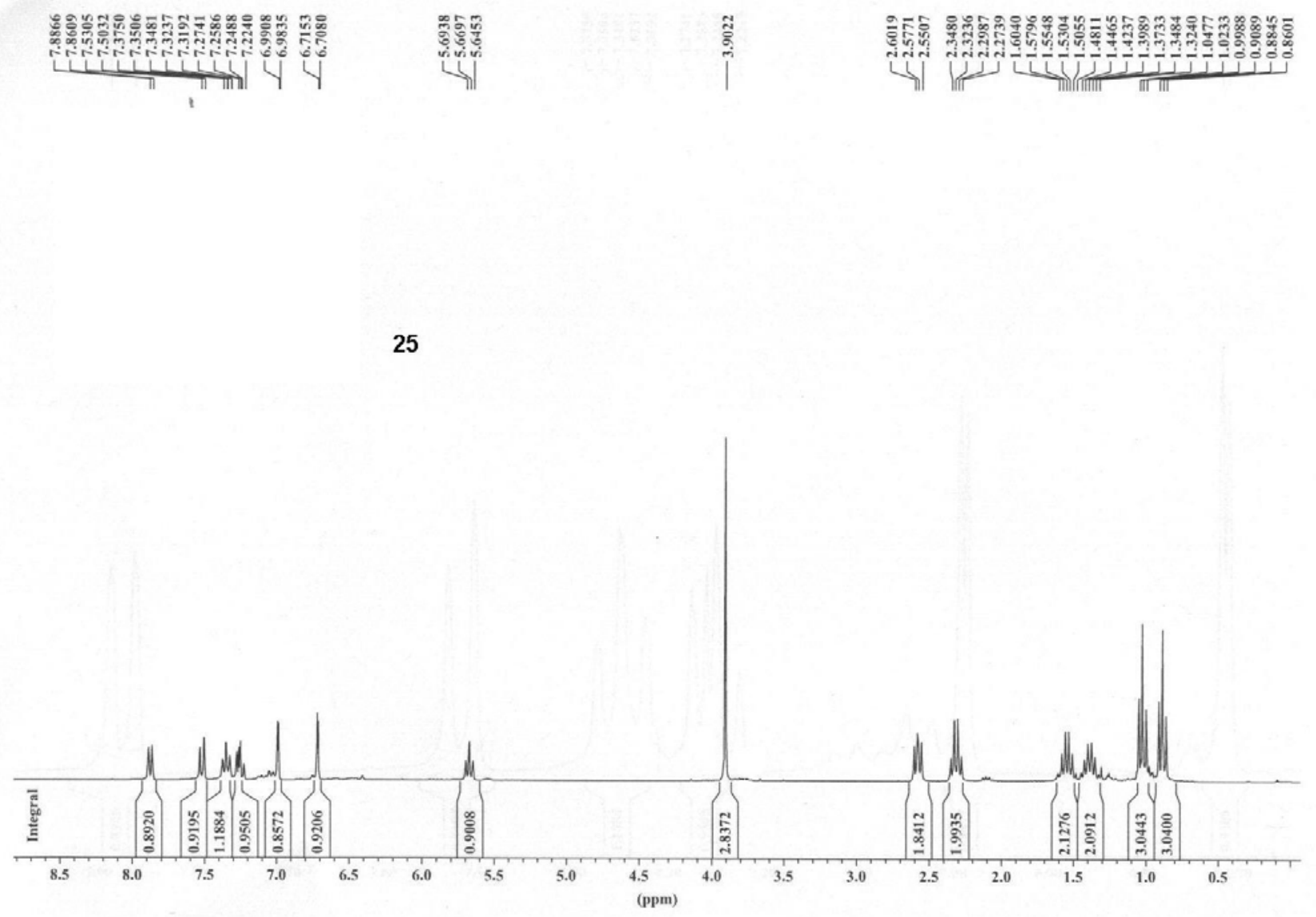


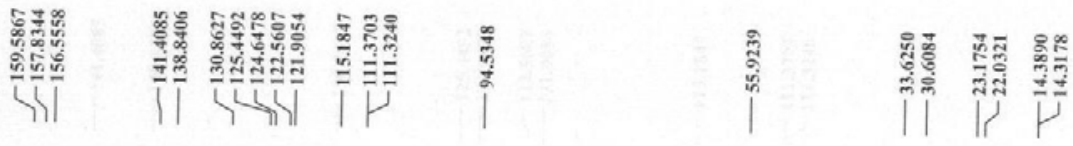

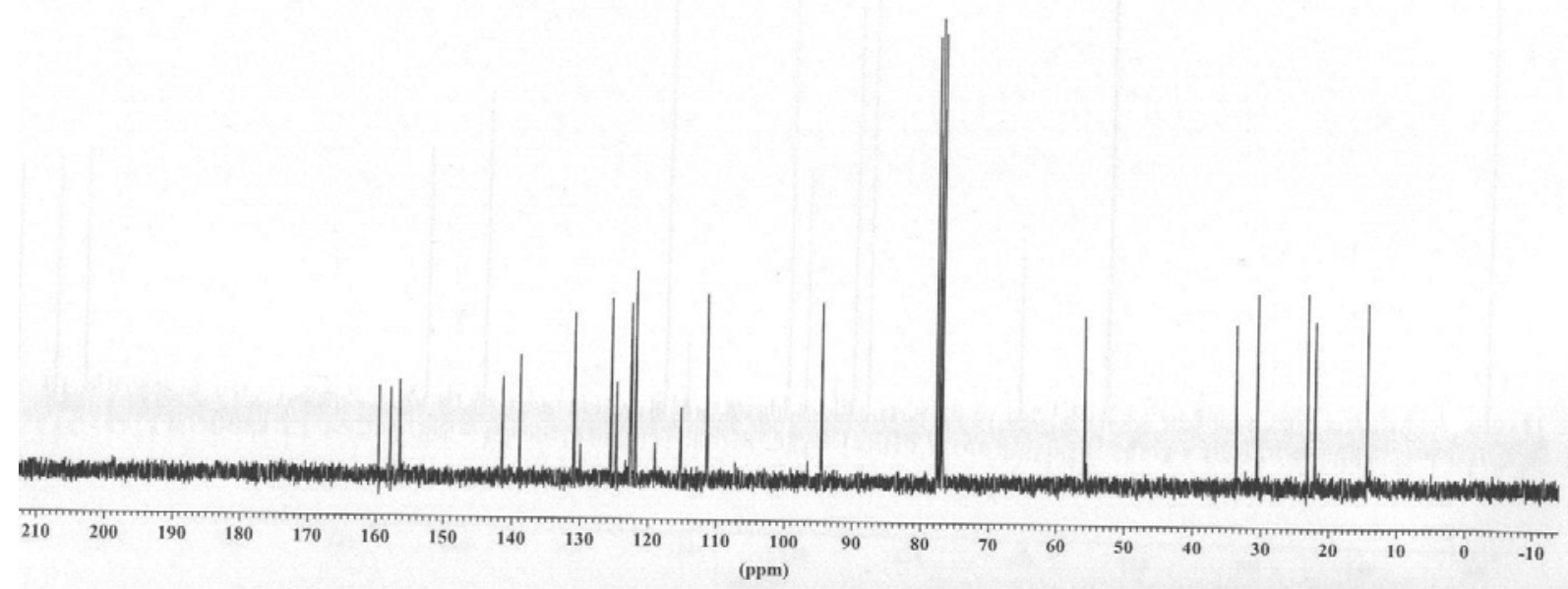


ZJ514

STANDARD 1 H OBSERVE

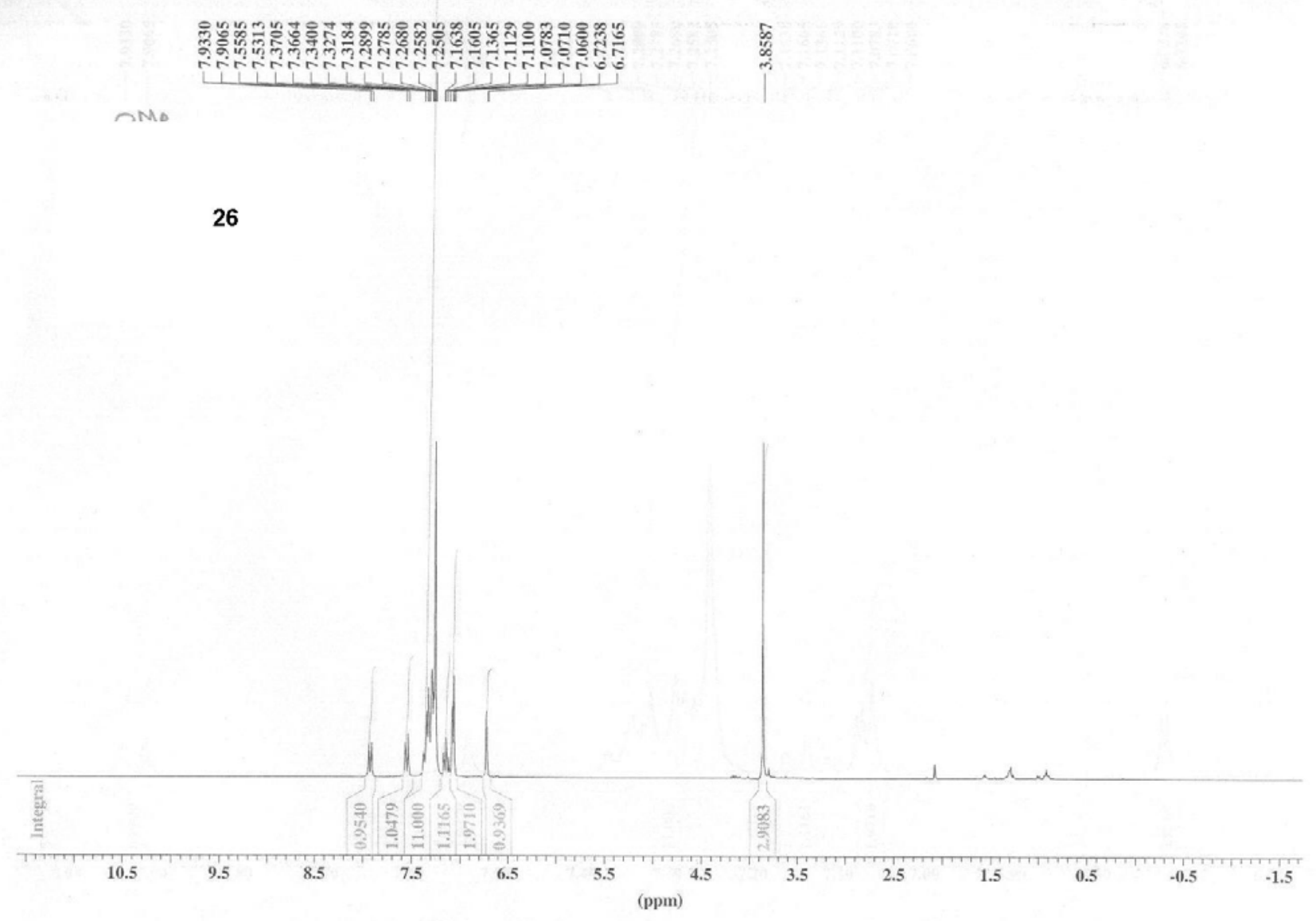




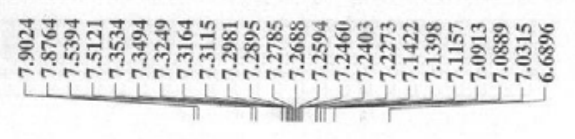

26d

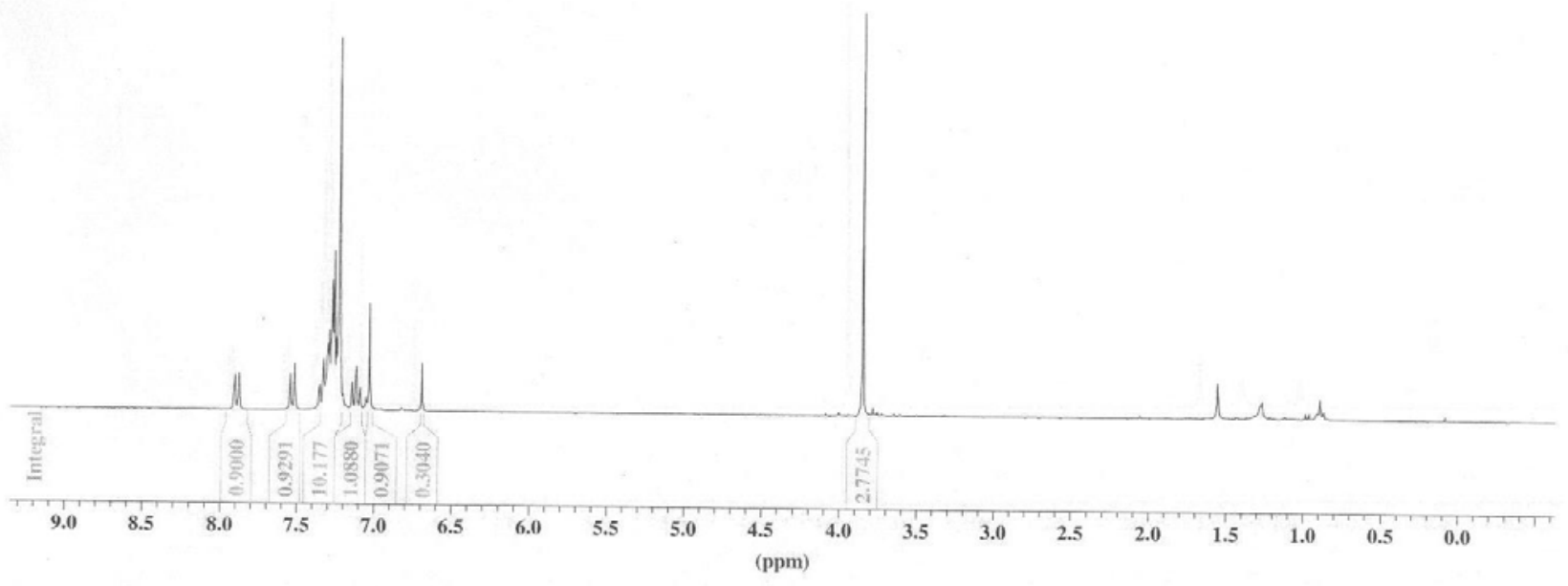

111111 11:2111: 


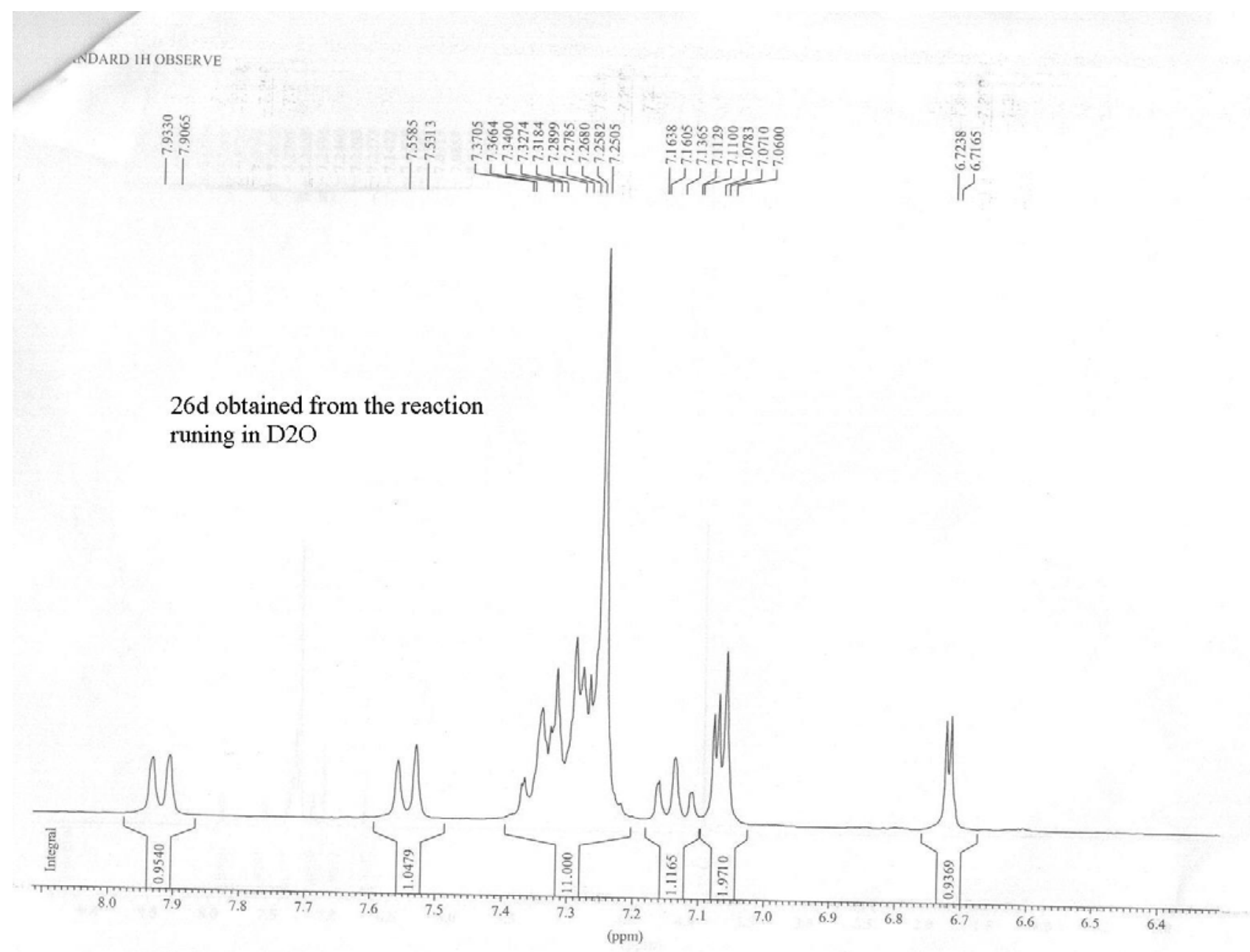

$11111+11=1111$ 
$\iint_{\text {STANDARD 1H OBSERVE }} 2 J 514$
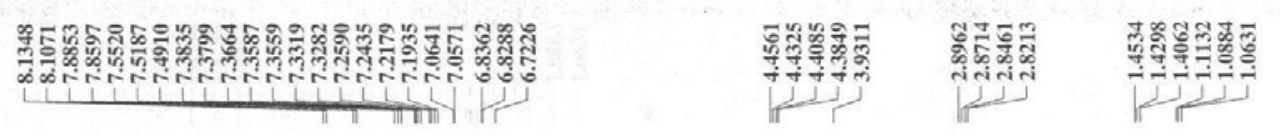

$27 a+27 b$

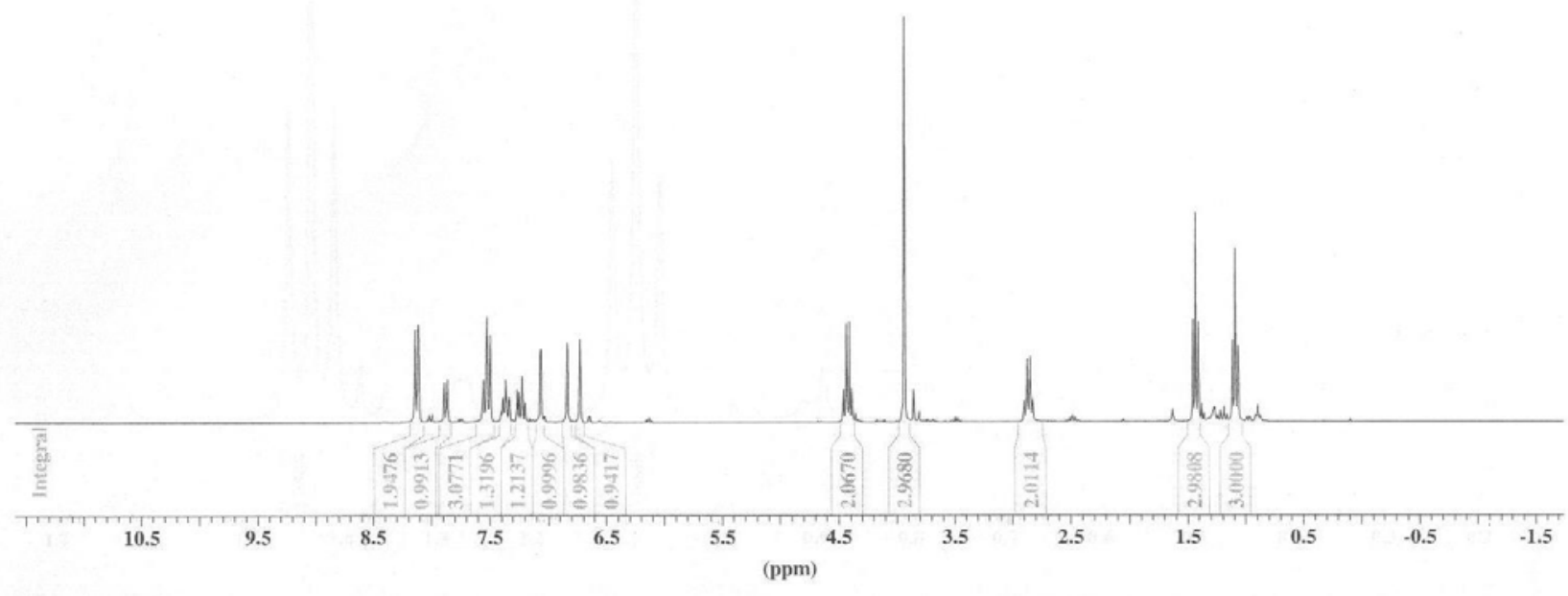


$27 a+27 b$

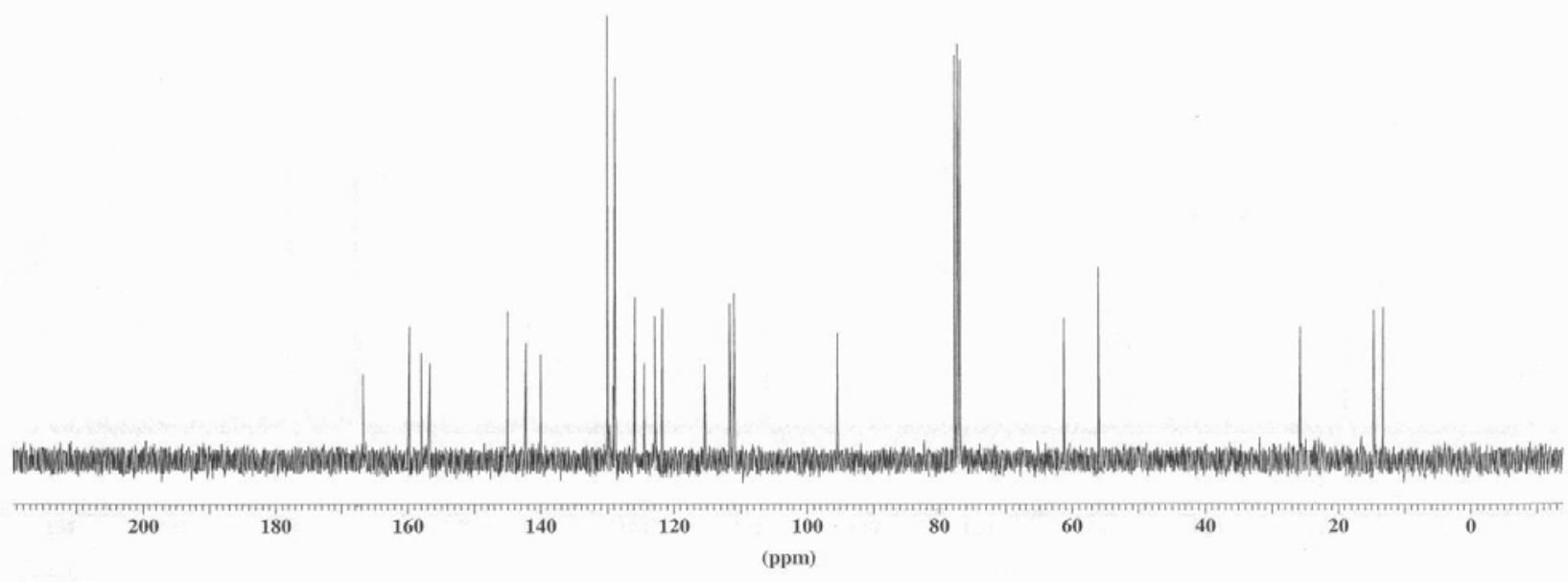


$28 a+28 b$

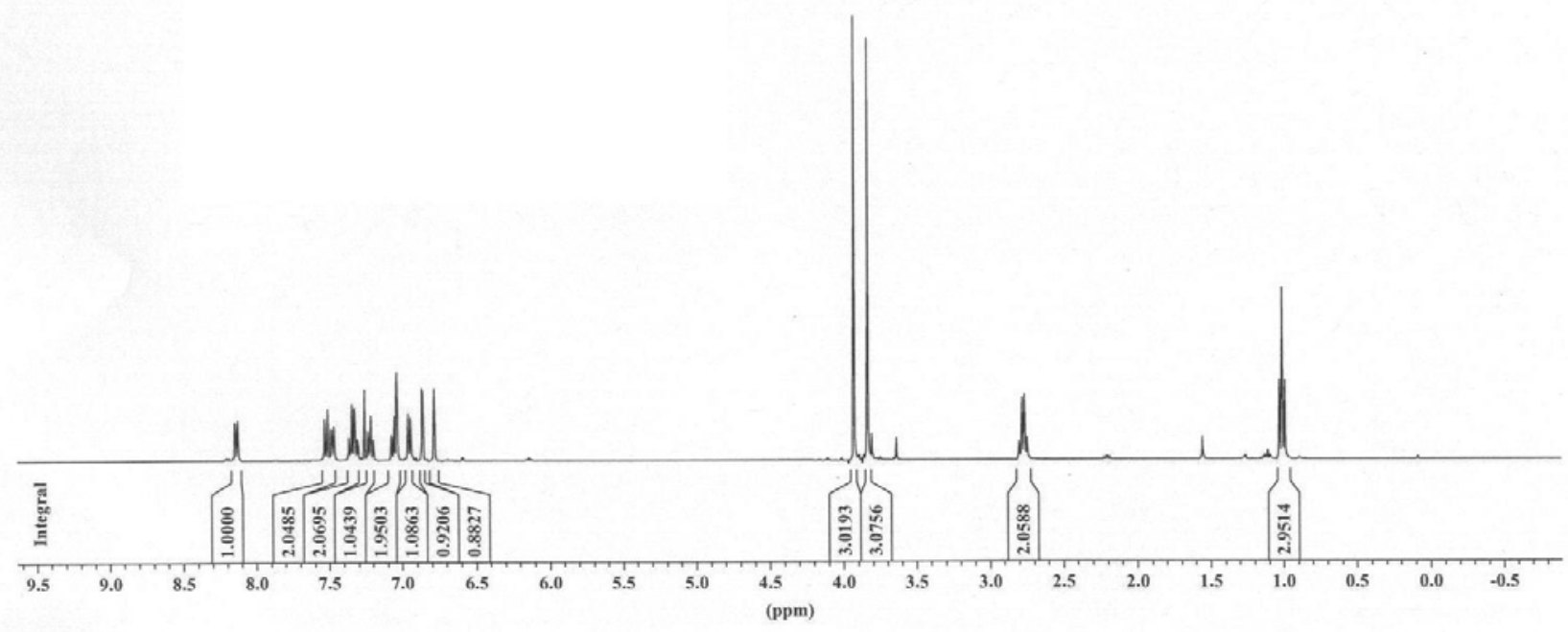




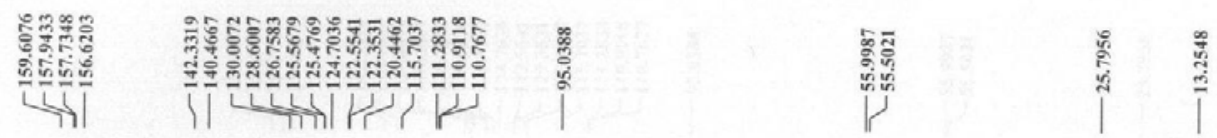

$28 a+28 b$

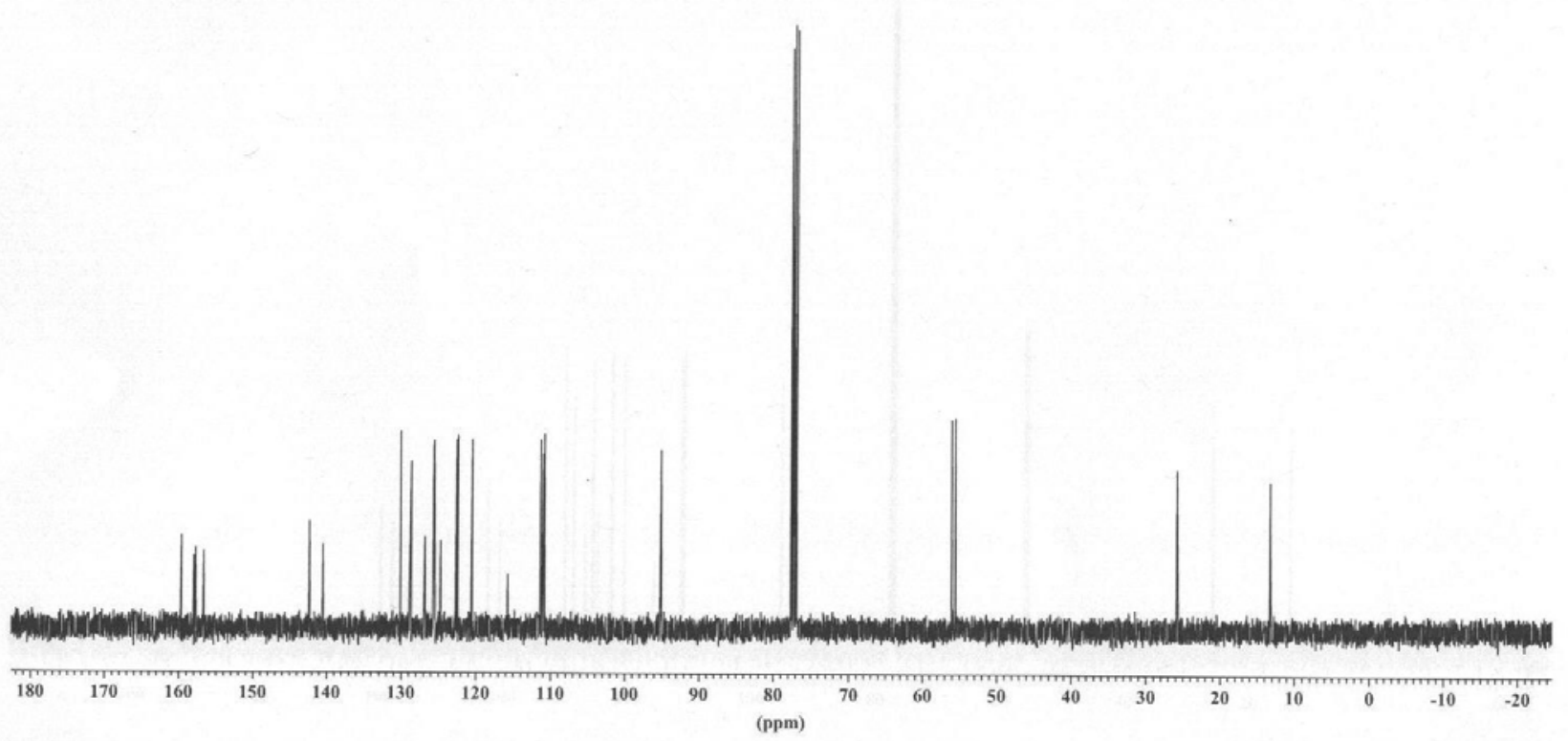

